\title{
LUMINESCENCE DATING: LABORATORY PROCEDURES AND PROTOCOLS
}

\author{
ANN G. WINTLE* \\ Institute of Geography and Earth Sciences, University of Wales, Aberystwyth SY23 3DB, U.K.
}

\begin{abstract}
In the last 30 years, f:-om 1967 to 1997, the use of luminescence signals from naturally occurring minerals has gone though a major metamorphosis, from thermoluminescence (TL) dating of pottery to optically stimulated luminescence (OSL) dating of sediments. Laboratory procedures for dating sediments have been adapted from those for pottery and new procedures have been developed as the need arises.

The majority of sediment dating applications are carried out on quartz and potassium-rich feldspars and the general characteristics of the TL and OSL signals from these minerals are reviewed. For sediments some new problems were encountered, with some grains perhaps not being completely bleached at deposition. For OSL signals there is no simple procedure for the selection of a thermally stable signal, as there had been in the case of pottery.

Many different laboratory protocols have been developed as our understanding of the fundamental behaviour of luminescence signals from quartz and feldspar has improved. These protocols are explained and discussed, giving the advantages and disadvantages of each procedure as applied to different types of sediment.

This review is presented as a gzuide to the selection of the most appropriate procedure for a particular dating application. (C) 1998 Elsevier Science Ltd. All rights reserved
\end{abstract}

\section{INTRODUCTION}

\subsection{Natural luminescence dosimeters}

Luminescence signals from quartz and feldspar grains have been used over the last 30 years to produce dates for heated materials, ranging from pottery and burnt flint (Roberts, 199\%) to lava-baked sediments (Forman et al., 1994b) and unheated sediments (Prescott and Robertson, 1997). The ages obtained range from a few tens of years (Ollerhead et al., 1994) to almost a million years (Huntley et al., 1993a, 1994). In the first dating studies, pottery dating was carried out using the thermoluminescence (TL) signal generated when the grains were heated following irradiation in thei: burial environment. Using heat to eject the charge from traps in the crystal lattice was clearly an appropriate procedure for materials which had their geologicallyproduced TL removed by heating at the event of interest. Once the TL signal from unheated quartz and feldspar grains was found to be reduced by exposure to sunlight, the potential for TL dating of sediments was explored (Wintle anc. Huntley, 1979). It was observed that modern samples had a finite TL signal due to the release of electrons from lightinsensitive traps and it was self-evident that heating previously unheated mineral grains was not the most appropriate measurement procedure. However, it was not until the mid-1980s that the use of light to eject light-sensitive electrons became a reality (Huntley et al., 1985).

Optically stimulated luminescence (OSL) was first observed using the $514.5 \mathrm{~nm}$ (green) light from an argon ion laser (Huntley et al., 1985). The success of this approach depended on the use of glass filters in the detection system which passed violet light from $360-420 \mathrm{~nm}$ whilst preventing the stimulationwavelength photons reaching the photomultiplier. One of the filters used is the Corning 7-59, already used in many TL dating studies. It was possible to observe OSL from both quartz and feldspars, since spectral measurements have shown that many feldspars emit luminescence in the violet to blue (390$440 \mathrm{~nm}$ ) (Huntley et al., 1988a) and that quartz OSL has an emission band in the near UV to violet (360-420 nm). As with TL, the OSL signals can be increased by adding laboratory dose and can be decreased by exposure to light. Optical stimulation of modern samples (Huntley et al., 1985; GodfreySmith et al., 1988) gave a zero signal, thus indicating that only electrons in light-sensitive traps were being observed.

Studies on feldspars, using a light source from which near infrared wavelengths could be used for stimulation, demonstrated that another luminescence signal could be observed from aluminosilicates (Hütt et al., 1988). This infrared stimulated luminescence (IRSL) allowed many laboratories to

*To whom all correspondence should be addressed. 
enter the field of optical dating, as a suitable stimulation source could be constructed from inexpensive IR emitting diodes (Poolton and Bailiff, 1989; Spooner and Franks, 1990). It can be seen from Fig. 6 of Bøtter-Jensen (1997) that stimulation at around $850 \mathrm{~nm}$ enables the observation of a much wider range of visible wavelengths than is possible for the $514.5 \mathrm{~nm}$ stimulation; in particular a Schott BG-39 filter is used in front of the photomultiplier tube since it absorbs the infrared stimulation at $850 \mathrm{~nm}$, but has almost $101 \% \%$ transmission at blue/ green wavelengths. Pure quartz does not appear to give a significant luminescence signal under IR stimulation (Short and Huntley, 1992), but purified sedimentary quartz from dune ridges in Australia has been shown to give measurable IRSL signals (Huntley et al., 1993b). The IRSL signal was interpreted as being derived from micro-inclusions within the quartz grains. Godfrey-Smith and Cada (1996) reported a weak IRSL signal for quartz extracted from sediments, but their measurements of the micro-inclusion area and composition led them to conclude that there were insufficient microinclusions to give rise to the observed signal. They concluded that their sigral, although showing a stimulation peak at $840 \mathrm{nrn}$, probably originated in the quartz lattice.

The desire to measure OSL from quartz using a relatively inexpensive ligh: source was satisfied by the use of a filtered halogen light. The broad stimulation spectrum (Fig. 8 cf Bøtter-Jensen, 1997) is achieved using a combination filter pack selected to produce the best signal-to-noise value (BøtterJensen and Duller, 1992). This green light stimulated luminescence (GLSL.) can be observed in the near-ultraviolet part of the spectrum using a Hoya U-340 filter (Fig. 8 of Bøt ter-Jensen, 1997).

These stimulation and detection systems, with minor variations in precise stimulation sources and filter combinations, form the technical background for the family of lumirescence dating methods. Dating is achieved by comparing the natural luminescence signal with those resulting from the administration of known aboratory doses. This comparison allows the equivalent dose, $D_{\mathbb{E}}$, to be determined; it is sometimes designated $E D$. The equivalent dose is the arnount of laboratory radiation dose that would be required to produce the natural luminescence signal. It is also known as the palaeodose, $P$. This paper examines the laboratory procedures which must be applied in order that the luminescence signals observed may be used to obtain correct and meaningful ages. Since the most important technique developments in the last 10 years have been in the field of sediment dating, this paper is primarily concerned with procedures and protocols related to this topic.

\subsection{Mineral separation}

Quartz and feldspar are widespread in the geological environment, and often occur in sediments as sand-sized, or finer, grains. In the parent rocks in which they were formed, they may occur as larger crystals (e.g. in granites) or as fine grains (e.g. in the matrix of a lava). Erosion by surface processes, e.g. the action of wind, water or ice, mechanical shock or chemical weathering, causes grains to break off from the parent rock. The grains will be further reduced in size during transport, e.g. by wind or water. Grains containing more than one mineral type will break along the boundary and crystals of a single mineral type will break along cleavage planes. The largest and most angular grains will be found close to their source bedrock and will not have been transported very far, e.g. close to an ice mass or in high energy beaches. Finer grains predominate in aeolian deposits, such as loess, and make up the inorganic component of deep sea sediments.

Grain sizes range from coarse sands (up to $5 \mathrm{~mm}$ in diameter) to clay-sized grains, which by definition are less than $4 \mu \mathrm{m}$ diameter. A range of grain sizes from $500 \mu \mathrm{m}$ down to $2 \mu \mathrm{m}$ was recognised in early TL studies on pottery and the effect on the microdosimetry was acknowledged (Aitken, 1985). This resulted in two laboratory techniques being developed - the fine grain technique, in which the grains in the 2-8 $\mu \mathrm{m}$ range (and for sediments $4-11 \mu \mathrm{m}$ ) are selected, and the inclusion technique, in which fine sand-sized grains $(90-125 \mu \mathrm{m})$ were extracted and purified using mineral separation techniques.

Grain size selection is carried out after the sample is disaggregated either mechanically or chemically. Gentle crushing is used to cause breaks between mineral grains in pottery or volcanic rocks. Cemented sediments can be broken down by gentle acid treatment (usually dilute $\mathrm{HCl}$ ). Grain size selection is then achieved by wet or dry sieving in the case of sand-sized grains or by settling in a liquid column according to Stokes' Law for fine grains. Further details can be found in the book by Aitken (1985) or individual dating papers.

The fine grains can be considered to receive the full alpha dose from elements in the decay chains of uranium and thorium in their environment, since the grain diameter is much smaller than the average alpha particle range in a silicate $(\sim 20 \mu \mathrm{m})$. The fine sand-sized grains will be mainly of quartz and feldspar, and, with a grain diameter around $100 \mu \mathrm{m}$, will receive alpha radiation from their environment only in the outer part of the grain. This contribution can either be calculated or eliminated by etching away the outer surface of the grain with HF. However, it should be noted that SEM studies have indicated that etching does not always proceed isotropically and preferential erosion along cleavage planes may occur (Bell and Zimmerman, 1978). 
Mineral separation techniques car be easily performed on sand-sized grains, $100 \mu \mathrm{m}$ or larger, using mineral-specific properties, such as density, chemical resistance or magnetic properties. Quartz is heavier than any feldspars, and usually has a density greater than $2.62 \mathrm{~g} \mathrm{~cm}^{-3}$. Potassium-rich feldspars are the lightest, tending to have densities in the range $2.58-2.54 \mathrm{~g} \mathrm{~cm}^{-3}$. Plagioclase feldspars tend to have densities between 2.58 and $2.62 \mathrm{~g} \mathrm{~cm}^{-3}$. Separation procedures are usually performed using liquids with densities of 2.62 and $2.58 \mathrm{~g} \mathrm{~cm}^{-3}$. Density separation for TL dating was first described by Mejdahl (1985) using heavy liquids made by mixing tetrabromoethane and dipropylene glycol. By 1988 these liquids had been replaced (for health reasons) by aqueous solutions of heavy inorganic salts, such as sodium polytungstate (for example as used by Rhodes, 1988; Godfrey-Smith et al., 1988).

Since the minerals cover a range of densities, such separations are less than perfect; this can be confirmed by X-ray diffraction on the separates (Godfrey-Smith and Cada, 1996). However, the quartz fraction can be cleaned of feldspar grains by etching in concentrated HF and then re-sieving to remove any grains which have been made significantly smaller. The purity of the et:hed grains can be monitored by making an IRSL measurement (Spooner and Questiaux, 1989; Smith et al., 1990b); quartz containing micro-inclusions of feldspar will always give a small IRSL signal (Huntley et al., 1993b).

Feldspar grains will always contain grains with some variation in composition, as well as feldspars of different crystal structure. In dating studies, the separates are monitored with regard to their potassium content, either by chemical techniques, such as flame photometry or atomic absorption spectrometry, or by beta counting, based on the assumption that the natural beta emission of grains with a density under $2.58 \mathrm{~g} \mathrm{~cm}^{-3}$ is dominated by that from ${ }^{40} \mathrm{~K}$. Although some K-feldspar separates have $K$ contents of $11-13 \%$ (Mejdahl, 1989), values of $8-10 \% \mathrm{~K}$ are commonly reported (Ollerhead et al., 1994). These are somewhat less than the theoretical maximum of $14.0 \% \mathrm{~K}$ calculated for a potassium aluminium silicate with a ciemical formula $\mathrm{KAlSi}_{3} \mathrm{O}_{8}$. Ollerhead et al. (1994) used a scanning electron microscope (SEM) to obtain the $\mathrm{K}$ content of individual grains in such a seprarated fraction. They concluded that $90 \%$ of their grains were $\mathrm{K}$ feldspars with a more likely $\mathrm{K}$ content of $12 \pm 1 \%$. A similar SEM study for sands from a tsunami deposit indicated that only about $50 \%$ of the grains in the separate were K-feldspars (Huntley and Clague, 1996); the values, calculated from this fraction and the chemically-determined $K$ content of the separate, ranged from $11-14 \%$. These values were then used for the age calculation of the samples, on the assumption that the IRSL was derived entirely from the $\mathrm{K}$-feldspar grains. RM 27;5-6-F
Measurements on a wider range of sediments have led Huntley and Baril (1997) to suggest use of a K content of $12.5 \pm 0.5 \%$ for the dating of the K-feldspar separate by IRSL. For large sand-sized grains from some environments, the internal beta dose rate from ${ }^{40} \mathrm{~K}$ is the dominant contributor to the natural dose rate.

The wide variation in composition and original source of mineral grains in sediment, or pottery or heated rocks, must be borne in mind when results of particular experiments are reported and general conclusions drawn. When contemplating a dating application in a new geographic area, or relating to a new depositional environment, an individual experimental programme may need to be devised to check the applicability of particular laboratory procedures.

\section{LUMINESCENCE SIGNALS FROM MINERALS}

\subsection{Quartz TL peaks}

Quartz exhibits a number of TL peaks when irradiated grains are heated from room temperature to $500^{\circ} \mathrm{C}$. Two peaks above $300^{\circ} \mathrm{C}$ were observed for sand-sized grains (quartz inclusions) extracted from coarse pottery by sieving and etching in concentrated HF (Fleming, 1970). The preferred peak for dating was observed at $375^{\circ} \mathrm{C}$ [Fig. 1(a)], for a heating rate of $\sim 5^{\circ} \mathrm{C} / \mathrm{s}$. A peak occurring on the lower shoulder of this peak, around $325^{\circ} \mathrm{C}$ [Fig. 1(b)], was found to show an unreliable dose response and was termed "malign" (Fleming, 1970). Another peak which attracted attention for dating pottery was only found in laboratory-irradiated samples; it occurred at about $110^{\circ} \mathrm{C}$ and became the basis of the "pre-dose dating method" when it was demonstrated that the peak height could be used to monitor dose-dependent sensitivity changes which were observed after heating to $500^{\circ} \mathrm{C}$ (Fleming, 1973).

These peaks, and others, are also observed in unheated quartz grains extracted from sedimentary deposits. Figure 1(c) shows the natural TL from a typical Australian sedimentary quartz (Wintle and Murray, in press). A peak at $110^{\circ} \mathrm{C}$ can be induced by laboratory irradiation, just as for quartz from pottery. The suite of peaks observed, and their relative intensities, can be altered by the choice of detection filter; this arises from the three main emission bands in quartz, as discussed by Krbetschek et al. (1997). Natural quartz has two emission bands, in the blue $(460-480 \mathrm{~nm})$ and in the orange (610$630 \mathrm{~nm}$ ) as shown in Fig. 2(a) for selected temperature ranges above $300^{\circ} \mathrm{C}$ (Huntley et al., 1988a). Lower temperature peaks resulting from laboratory irradiation also show an emission band in the near UV to violet $(360-420 \mathrm{~nm})$, and it is this waveband which is observed for OSL (see Section 2.2). 

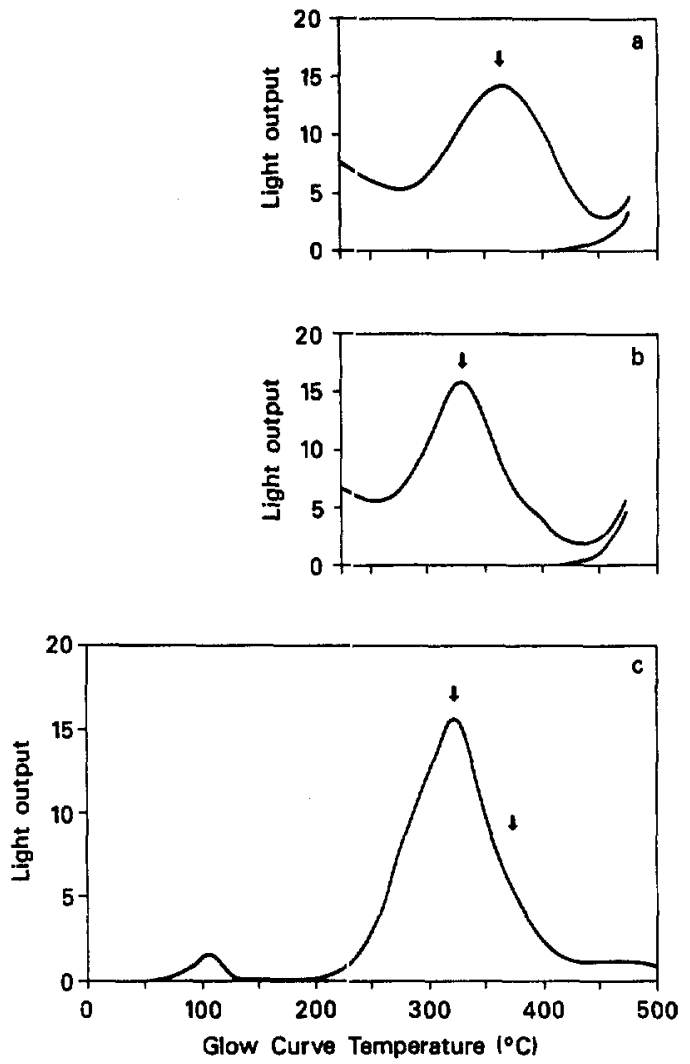

Fig. 1. (a) and (b) TL glow curves for quartz extracted from pottery measured at a teating rate of $20^{\circ} \mathrm{C} / \mathrm{s}$ showing dominant peaks at 375 and $325^{\circ} \mathrm{C}$ (from Fleming, 1970). TL was observed using a Corning 5-60 and a ChancePilkington HA3 filter and was for a $5.5 \mathrm{~Gy}$ dose given after heating to $500^{\circ} \mathrm{C}$. (c) Natural TL measured at a heating rate of $5^{\circ} \mathrm{C} / \mathrm{s}$ for WIDGB, $D_{\mathrm{E}}=58 \pm 6 \mathrm{~Gy}$, a sedimentary quartz from Widgingarra, Australia (from Wintle and Murray, in press). TL was observed using two Hoya U340 filters and an HA-3 filter after a 0.1 s green light exposure for normalization, which resulted in the PTTL peak at $110^{\circ} \mathrm{C}$.

Figure 3(a) shows the natural TL for another Australian quartz obtained using a Corning 7-59 filter and (inset) a combination of this filter and a Schott UG11 (Prescott and Fox, 1990). The $375^{\circ} \mathrm{C}$ peak can scarcely be seen on the high-temperature edge of the $325^{\circ} \mathrm{C}$ peak when the Schott UG11 filter is added. The two main quartz peaks above $300^{\circ} \mathrm{C}$ show different bleaching behaviour under sunlight, leading to them being described as the "slowly bleaching peak" (SBP) and the "rapidly bleaching peak" (RBP) (Franklin and Hornyak, 1990). Using the Corning 7-59 and Schott UGll filter combination for yet another Australian quartz, Spooner et al. (1988) showed the ease with which the $325^{\circ} \mathrm{C}$ peak could be bleached by light of $500 \pm 20 \mathrm{~nm}$ (Fig. 5), as discussed fur:her in Section 2.1.2.

2.1.1. $375^{\circ} \mathrm{C} T L$ peak: (SBP). The TL peak at $375^{\circ} \mathrm{C}$ has been used extensively for dating coastal dune sediment in Australia (Huntley et al., 1993a, 1994). It bleaches more slowly than the $325^{\circ} \mathrm{C}$ peak, but wind-blown grains and even sediments from major river systems (Page et al., 1996) are well bleached down to a non-reducible residual level by the high intensity sunlight incident on Australia.

Spectral studies by Scholefield et al. (1994) have shown that the $375^{\circ} \mathrm{C}$ peak emission for their Australian sedimentary quartz [Fig. 4(b)] has a maximum at about $480 \mathrm{~nm}$. This emission has been suggested by McKeever et al. (1985) to be $\mathrm{AlO}_{4}$ acting as a hole trap in the quartz lattice. The behaviour of this slowly bleaching peak has been studied by observing it with a green filter (Chance OGrl) in combination with the Chance HA3 heat-absorbing filter (Franklin et al., 1992). Any contribution from the $325^{\circ} \mathrm{C}$ rapidly bleaching peak could be removed by bleaching with green light, e.g. using the Chance OGrl filter (Franklin et al, 1992) or a Schott GG475 filter (Prescott and Fox, 1990) in front of the broad emission light source.

Trap depth studies indicate that this peak has high thermal stability $\left(E=1.66 \mathrm{eV}, \log _{10} S=13\right.$ and $\tau$ at $15^{\circ} \mathrm{C}>10^{8} \mathrm{y}$ ) (Aitken, 1985). However, it has been reported to saturate at relatively low doses and might thus be thought not to be useful for dating over $50 \mathrm{ka}$. However, this peak has been used most successfully in the dating of Australian beach ridges back to $500 \mathrm{ka}$ (Huntley et al., 1993a, 1994) where it shows a non-saturating response to laboratory and natural radiation (see Section 4.2) and where natural dose rates are very low $(\sim 0.5 \mathrm{~Gy} / \mathrm{ka})$.

More complex behaviour, which might limit the use of the $375^{\circ} \mathrm{C}$ TL peak for dating, has been reported by Hornyak et al. (1992) for a quartz from the Kalahari Desert. Following a green light bleach to remove the $325^{\circ} \mathrm{C}$ TL peak (Franklin and Hornyak, 1990), the TL was observed with either an ultraviolet filter (Schott UG11) centred at $380 \mathrm{~nm}$, or a green filter $(\mathrm{OGr} 1)$ centred at $480 \mathrm{~nm}$. A single peak was obtained, but it was shifted some $20^{\circ} \mathrm{C}$ lower when observed with the ultraviolet filter and was about half the magnitude (uncorrected for filter transmission characteristics or photomultiplier response). Kinetic analyses of the two peaks suggested a distribution of activation energies, each centred at $1.45 \mathrm{eV}$. A computer model was generated which used a single electron trap and two recombination centres. This interpretation accounted for the similarity of the isothermal decay curves obtained for each peak when held at 260 and $293^{\circ} \mathrm{C}$, when the data were normalised relative to the initial signal. Hornyak et al. (1992) discussed how this model might also affect the apparent growth curve shape when dose is added in the laboratory, giving rise to an age underestimation. In a subsequent dating study on sand from the Kalahari, Feathers (1997) has selected a narrower spectral region by using a combination of the Schott UGl1 and the Corning 7.59 filter. For their study of the coastal dune ridges, Huntley et al. (1993b, 1994) used two Corning 7-59 filters $(320-420 \mathrm{~nm})$, 
(a)

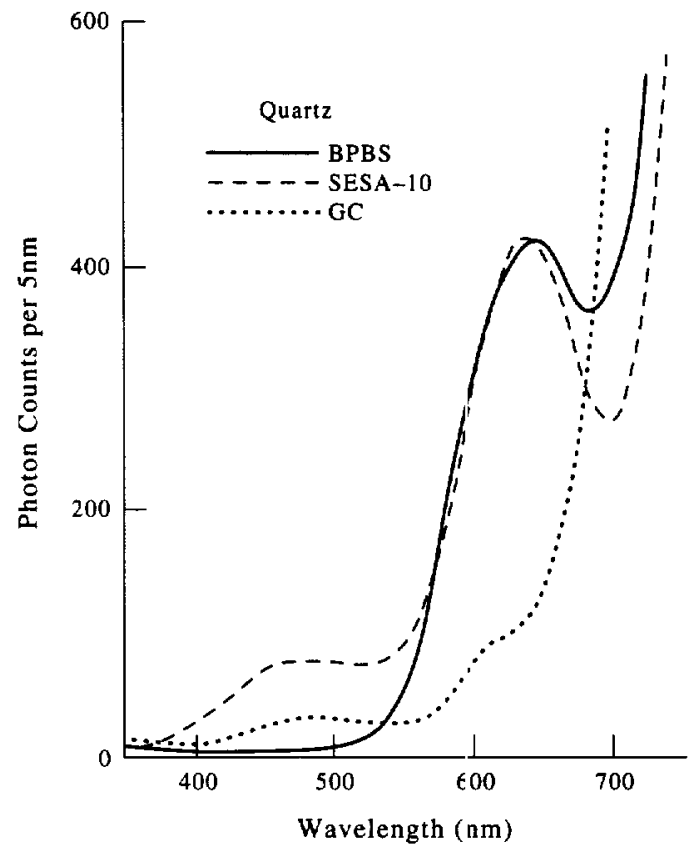

(b)

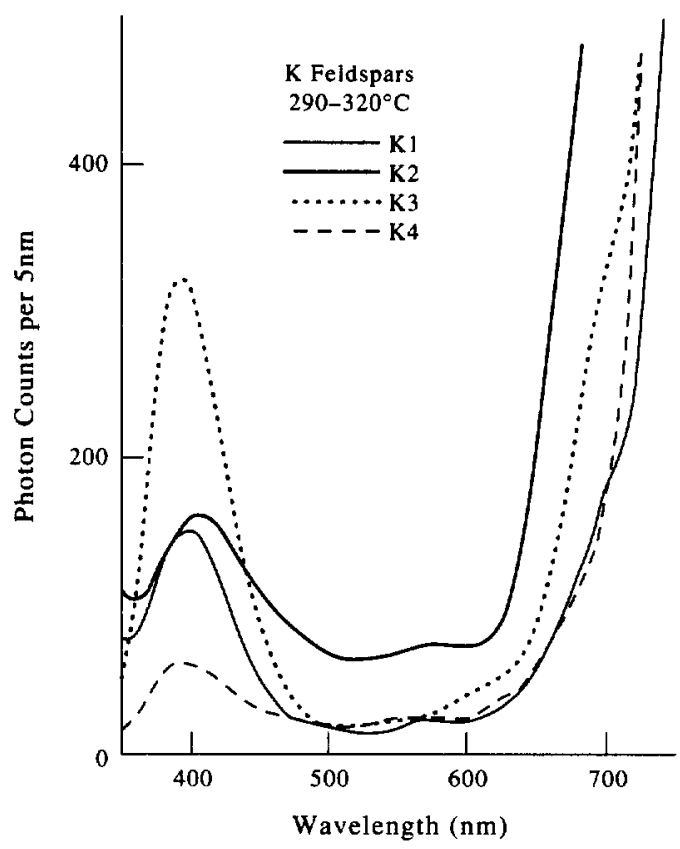

(c)

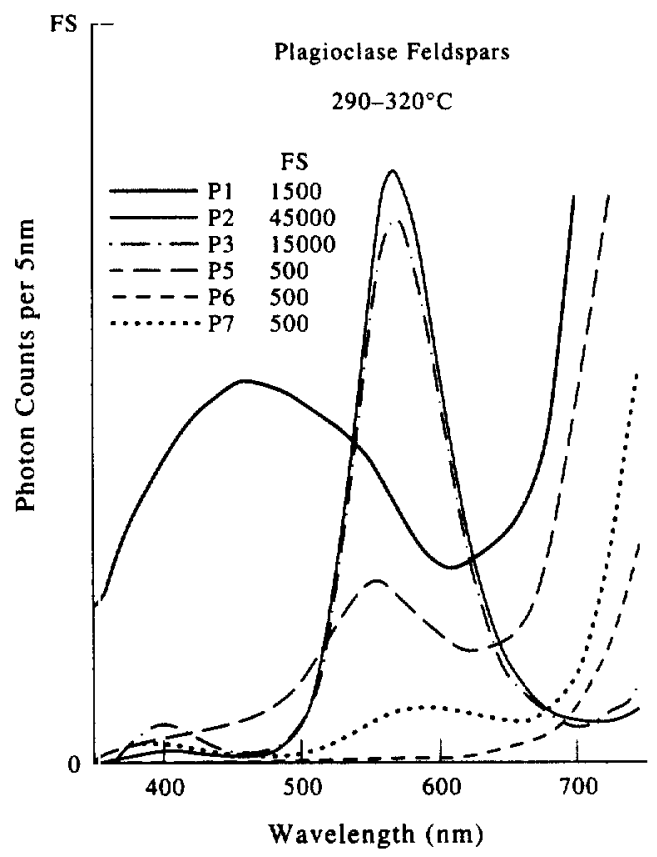

Fig. 2. TL emission spectra of examples of (a) quartz, (b) potassium-rich feldspars and (c) plagioclase feldspars (from Huntley et al, 1988a). The quartz samples were extracted from sediments. The steep rise measured above $650 \mathrm{~nm}$ is primarily incandescence, but feldspars $\mathrm{K} 2$ and $\mathrm{K} 4$ have significant emission in this wavelength region.

together with a heat-absorbing fiter, which would have passed part of the blue emission.

2.1.2. $325^{\circ} \mathrm{C} T L$ peak (RBP). The TL peak at $325^{\circ} \mathrm{C}$ has its emission peak at about $380 \mathrm{~nm}$ (Fox, 1990; Scholefield et al., 1994) [Fig. 4(c)]. The signal from this peak can be enhanced rilative to, but not totally separated from, the $375^{\circ} \mathrm{C}$ peak by using a detection filter pack made up of a Schott UG-11 and a Corning 7-59 filter which passes from 320 to $380 \mathrm{~nm}$ (Prescott and Fox, 1990; Franklin and Hornyak, 1990). The luminescence centre used by electrons giving rise to the $325^{\circ} \mathrm{C}$ peak had previously 


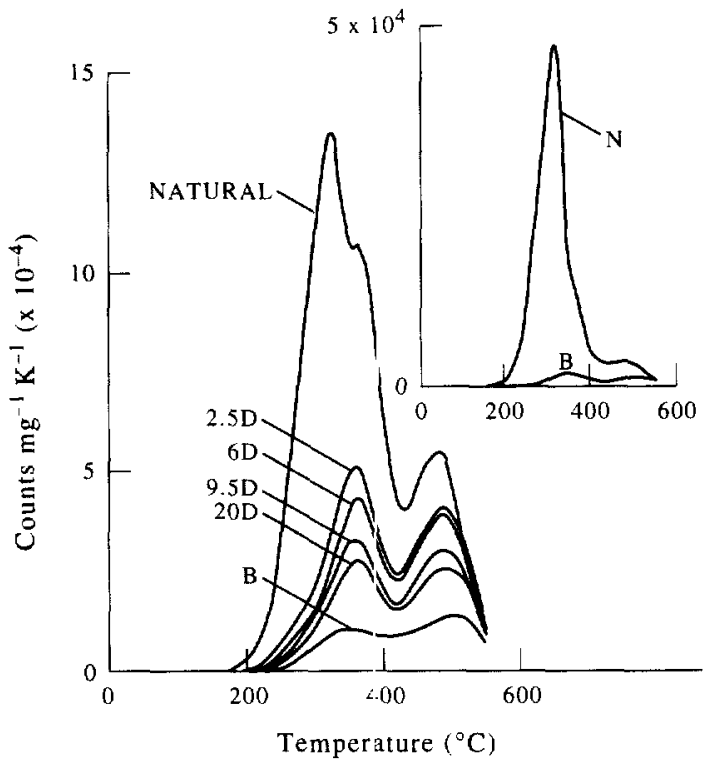

Fig. 3. Natural TL of unetched Australian sedimentary quartz from Puritjarra observed with a Corning 7-59 filter and inset with a combination of a Corning 7-59 and Schott UG-11 filters (from Prescott and Fox, 1990); also shown is the TL after sunlight bleaching for 2.5 to 20 days. In each case B is effect of 2 day bleach on etched quar:z.

been identified in quartz extracted from pottery as exhibiting thermal quench ng (Wintle, 1975). This refers to the increased probability of non-radiative de-excitation of the centre during the recombination process. This resulted in a correction procedure having to be applied, before the kinetic parameters were calculated from laboratory experiments based on the initial rise method $(E=1.69 \mathrm{eV}$, $\log _{10} S=14$, and $\tau$ at $15^{\circ} \mathrm{C} \sim 10^{8} \mathrm{y}$ ) (Aitken, 1985). These values suggest that the $325^{\circ} \mathrm{C}$ peak should be stable for dating up to $10^{6} \mathrm{y}$.

The $325^{\circ} \mathrm{C}$ TL peak ble:aches very rapidly, even at wavelengths as long as $500 \mathrm{~nm}$. Figure 5 shows that the natural $325^{\circ} \mathrm{C}$ signal is bleached very rapidly when exposed to $500 \pm 20 \mathrm{~nm}$ light for only $1 \mathrm{~min}$, when even exposure for $1200 \mathrm{~min}$ does not cause any reduction in the $375^{\circ} \mathrm{C}$ peak. Use of higher energy photons will bleach the $325^{\circ} \mathrm{C}$ peak even more rapidly.

2.1.3. $110^{\circ} \mathrm{C}$ TL peak (pre-dose peak). The TL peak which is found at just about $100^{\circ} \mathrm{C}$ for a heating rate of $5^{\circ} \mathrm{C} / \mathrm{s}$ is know/n as the $110^{\circ} \mathrm{C}$ peak. A peak in this region can be seen in all quartz, whether natural or artifcial (Petrov and Bailiff, 1995) provided that it has been irradiated less than a few hours before measurement [Fig. 3(a)]. The peak has a maximum ernission at about $380 \mathrm{~nm}$, corresponding to the $\mathrm{H}_{3} \mathrm{O}_{4}$ hole centre in quartz (Yang and McKeever, 1990).

The $110^{\circ} \mathrm{C}$ peak electron traps can be populated by irradiation or by photo-transfer. For instance, when quartz with a st:ong $325^{\circ} \mathrm{C}$ TL peak is exposed briefly to green light at room temperature (Wintle and Murray, in press) and then heated, phototransfer is observed, as in Fig. 1(c). The kinetic parameters of the $110^{\circ} \mathrm{C}$ TL peak are given as $E=0.98 \mathrm{eV}, \quad \log _{10} S \sim 13$ and $\tau$ at $15^{\circ} \mathrm{C} \sim 7 \mathrm{~h}$ (Aitken, 1985).

The response of the $110^{\circ} \mathrm{C}$ TL peak to a small test dose can be enhanced significantly by heating to temperatures above $200^{\circ} \mathrm{C}$. The process was explained by Zimmerman (1971) and Yang and McKeever (1990), and used to measure small doses received by ceramics (Bailiff, 1994).

2.1.4. Other TL peaks. Examination of the glow curve of laboratory irradiated sedimentary quartz shows that there are several other peaks; for example Fig. 6(a) shows peaks at 160 and $280^{\circ} \mathrm{C}$, the former also being rapidly bleached by exposure to light (Wintle and Murray, 1997). In their study of emission wavelengths from another Australian quartz, Franklin et al. (1995) reported rapidly bleaching peaks at $150-180^{\circ} \mathrm{C}$ and at $200-220^{\circ} \mathrm{C}$, having emission wavelengths of 392 and $410 \mathrm{~nm}$, respectively. Their spectral plots are shown in Fig. 6(b). They conclude that the peaks at 110,180 , 220 and $325^{\circ} \mathrm{C}$ all use the same luminescence centre, and its emission peak shifts to higher wavelengths as the temperature of the sample is increased.

\subsection{Quartz $O S L$}

OSL can be observed when irradiated quartz is exposed to light of any visible wavelength. Stimulation is usually achieved with either the $514.5 \mathrm{~nm}(2.41 \mathrm{eV})$ argon line or a broad band green light source based on a filtered halogen lamp. However, the efficiency of OSL production as a function of stimulation wavelength has been measured using other laser lines from the deep red $646 \mathrm{~nm}(1.92 \mathrm{eV})$ to the blue $458 \mathrm{~nm}(2.71 \mathrm{eV})$ and including 626,569, 530 and $488 \mathrm{~nm}$ (Ditlefsen and Huntley, 1994). A continuous stimulation spectrum from $420-650 \mathrm{~nm}$ (Bøtter-Jensen et al., 1994) has been obtained for a sedimentary quartz (Fig. 19 of Bøtter-Jensen, 1997). These measurements showed that OSL production is strongly dependent upon wavelength, with more energetic photons resulting in higher OSL intensities. This might be thought to result in different behaviour, depending upon whether a monoenergetic laser line is used or a broad light source, such as in the filtered light source of the Riso reader (Fig. 8 of Bøtter-Jensen, 1997). This was explored by Duller and BøtterJensen (1996) and no difference was observed. Murray and Wintle (1997) conclude that although the lamp gives a stimulation band from 420 to $550 \mathrm{~nm}$, it behaves as a stimulation source with an effective energy of $2.65 \mathrm{eV}(468 \mathrm{~nm})$. It is presumed that the main effect of decreasing stimulation wavelength is to increase the detrapping probability, and 
(a)

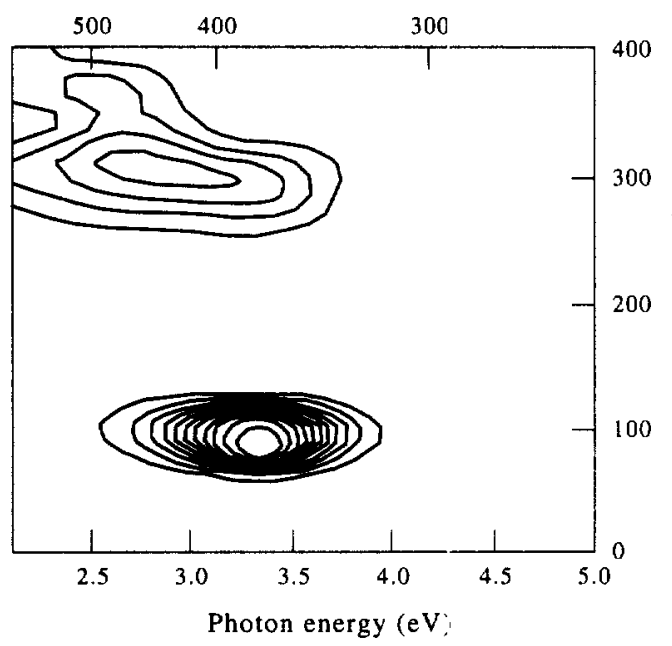

(b) Wavelength (nm)

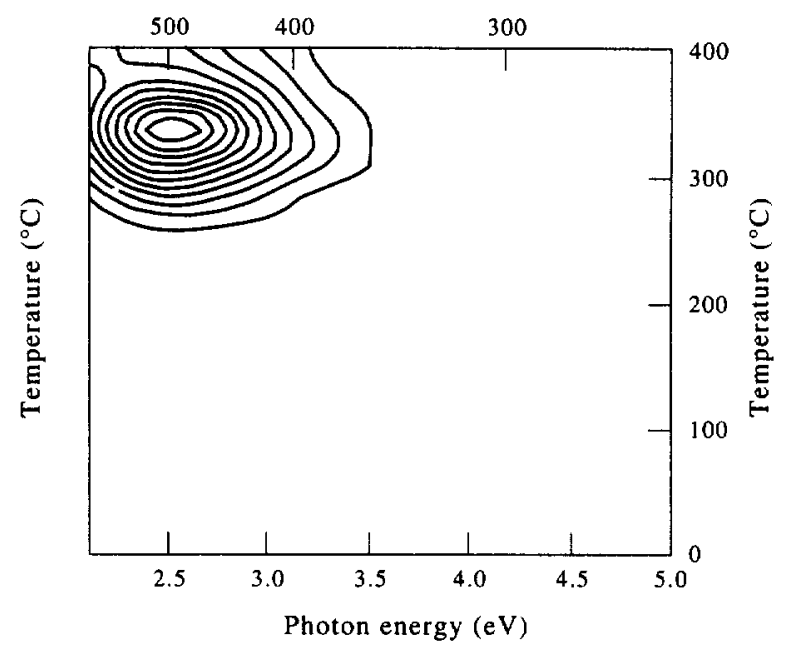

(c) Wavelength $(\mathrm{nm})$

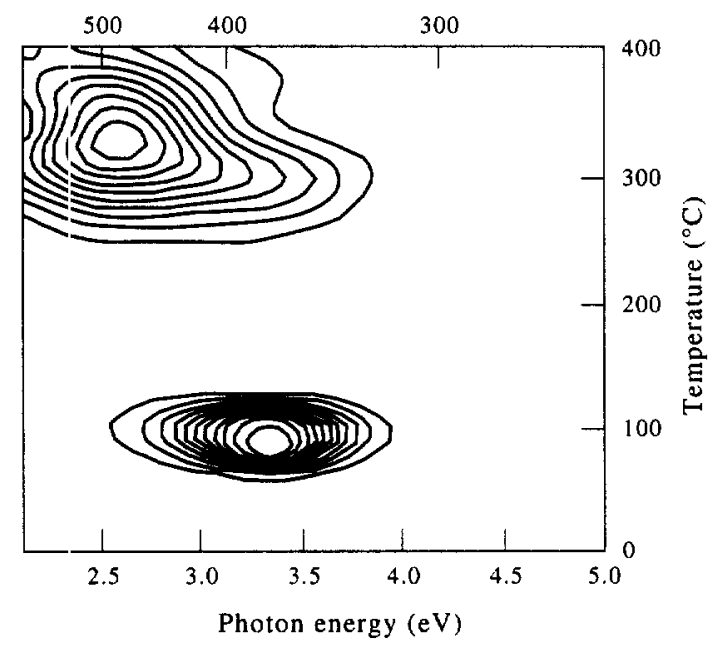

Fig. 4. Spectral measurements of the natural TL of Australian sedimentary quartz from Tennant Creek (from Scholefield et al., 1994) (a) preheated at $240^{\circ} \mathrm{C}$ for $60 \mathrm{~s}$ and given 1 Gy dose to activate the $110^{\circ} \mathrm{C}$ peak, (b) after bleaching in yelow light for $30 \mathrm{~min}$ and preheated at $240^{\circ} \mathrm{C}$ for $60 \mathrm{~s}$ and (c) the difference spectrum between the data in (a) and (b) to obtain the spectrum of the rapidly bleaching peak at around $325^{\circ} \mathrm{C}$.

thus the OSL production rate, rather than to access different (deeper) traps.

The emission spectrum for Australian dune sands has been observed using a stimulation wavelength of $647 \mathrm{~nm}$ from a krypton laser (Huntley et al., 1991). Figure 7(a) shows a single emission band centred on $365 \mathrm{~nm}$. This data point is marked on Fig. 6(b) (at $22^{\circ} \mathrm{C}$ ), and is in line with the trend of emission maxima of the rapidly bleaching $T L$ peaks.

The OSL signal has been extensively studied (see Spooner (1994b) and Huntley et al. (1996) for overviews) and used in a variety of dating applications (see reviews by Prescott and Robertson (1997) and Roberts (1997)). No concensus has been reached concerning the long-term stability of the OSL sig- nal. OSL signals have been observed for quartz grains ranging from a few years (Murray et al, 1995) to almost a million years old (Huntley et al., 1985). However, the OSL signal of a laboratory irradiated quartz has been observed to decay substantially on storage over a few weeks at ambient temperatures (Godfrey-Smith, 1994). Hence the OSL signal appears able to be generated from at least one unstable and one stable trap. Using isothermal decay studies at elevated temperatures, Smith et al. (1990a) considered the more geologically stable signal to be derived from a single electron trap $\left(E=1.84 \mathrm{eV}, \log _{10} S=15.3\right.$ and $\tau$ at $20^{\circ} \mathrm{C} \sim 600 \times 10^{6} \mathrm{y}$ ). A similar thermal stability has been reported by Wintle and Murray (in press) who concluded that a single electron trap explained over 


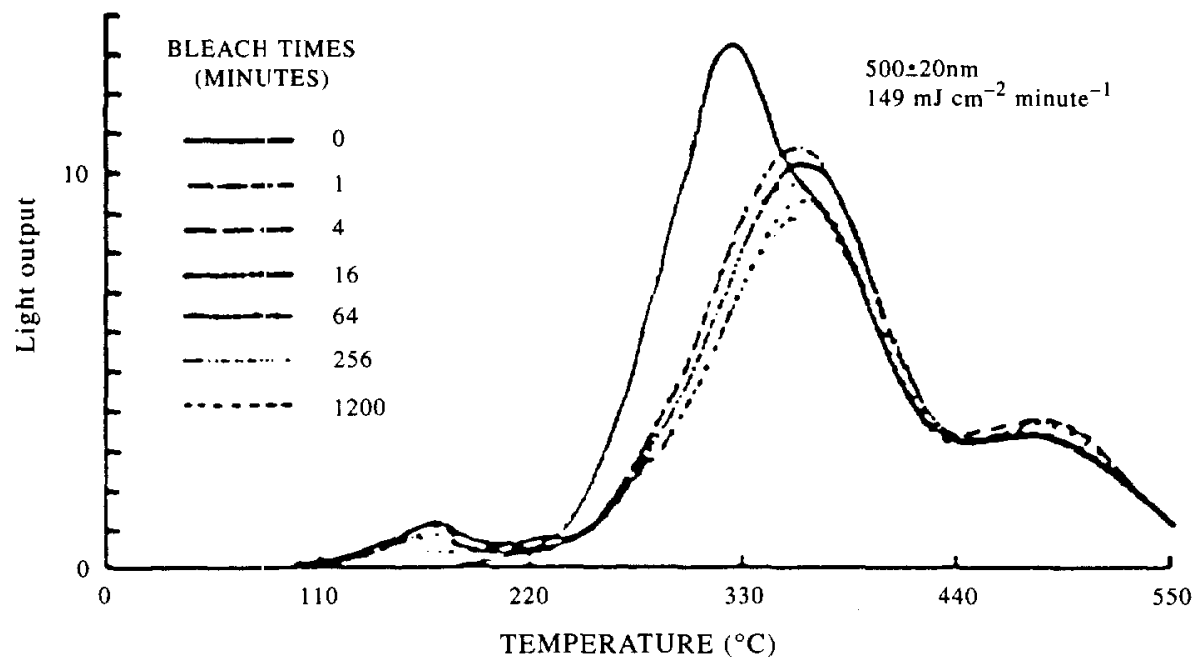

Fig. 5. Natural TL of Australian quartz from Lake Woods observed using HA3 and a Corning 7-59 filter (from Spooner et al., 1988) as measured after various lengths of exposure to light in a narrow wavelength band centered at $500 \mathrm{~nm}$.

$99 \%$ of their natural OSL from a 35000 -year-old sediment. These reports conflict with that of Huntley et al. (1996), who found the natural OSL of a very old sample to require four summed exponentials to explain their isothermal decay data. Their data, and those of Smith et al. (1990a) and Wintle and Murray (in press) are given in Table 1. More isothermal decay experiments on samples of different ages and levels of saturation are clearly required to establish which interpretation is appropriate.

\subsection{Feldspar TL peaks}

Many potassium feldspars extracted from sediments also exhibit two rain TL peaks at around 280 and $330^{\circ} \mathrm{C}$ for a heating rate of $2{ }^{\circ} \mathrm{C} / \mathrm{s}$ (Mejdahl, 1988a) (Fig. 3 of Bøtter-Jensen, 1997). TL emission spectra of various alkali and plagioclase feldspars were obtained by Huntley et al. (1988a,b) and are reproduced in Fig. 2(b,c). Figure 2(b) shows violet to blue emission (390-440 nm) for some K-rich feldspars and Fig. 2(c) shcws yellow-green emission (560-570 nm) for some plagioclase feldspars. More complex TL spectral behaviour has been reported for well-characterised thin sections of feldspars (Garcia-Guinea et al., 1996; Krbetschek et al., 1997) but no simple rela:ionship with structure has been found.

Effects of sunlight exposure have been reported for TL of feldspars measured using a Corning 7-59 filter combined with an HA-3 (Robertson et al., 1991, 1993). Data were crbtained for samples whose emission spectra had previously been obtained (Prescott et al., 1990; Prescott and Fox, 1993). A 16-h exposure reduced the natural TL to less than $10 \%$ of the initial value, but the bleaching rate had already slowed appreciably after the first $5 \mathrm{~h}$, with the lower temperature peaks bleaching more rapidly. Charge transfer was observed with PTTL peaks appearing in some samples (Robertson et al., 1991). The effects of sunlight bleaching were explored in more detail by Prescott et al. (1994) when the emission spectra of the same samples were observed after a 1-h sunlight exposure. They conclude that feldspars have no rapidly bleaching peak similar to the RBP peak in quartz, and hence the OSL signal from feldspars cannot be linked to a particular TL peak.

Significant reduction in the TL signal of feldspars by exposure to infrared has also been found (Duller, 1995a) but the reduction is not specifically related to a single TL peak. No link between IRSL and a particular TL peak has been established.

The trap depth studies of Strickertsson (1985) suggested that the $330^{\circ} \mathrm{C}$ TL had relatively high thermal stability $\left(>25 \times 10^{6} \mathrm{y}\right.$ for storage at $\left.10^{\circ} \mathrm{C}\right)$, but studies on feldspar grains separated from a Tertiary sand from Jutland $\left(10-20 \times 10^{6} \mathrm{y}\right)$ suggest a lifetime of about $0.8 \times 10^{6}$ y for a K-feldspar separate with $\mathrm{K}=10.5 \%$ (Mejdahl, 1988a), thus limiting its application to grains less than $0.5 \times 10^{6} \mathrm{y}$ old, at least for feldspars of Scandinavian origin. In a further study of Tertiary sands, Mejdahl (1989) measured the ratio of the saturation TL signal, assumed to be at an equilibrium value. Combining these ratios with the individual dose rates, he calculated lifetimes of about $0.7 \times 10^{6} \mathrm{y}$ for the $330^{\circ} \mathrm{C}$ peak and about $0.4 \times 10^{6} \mathrm{y}$ for the lower peak. Mejdahl (1989) gives an age equation which corrects for thermal fading and makes allowance for the different rates at which the saturation level is approached, i.e. owing to the different dose rates. For a sample of $100 \mathrm{ka}$, the uncorrected TL ages 


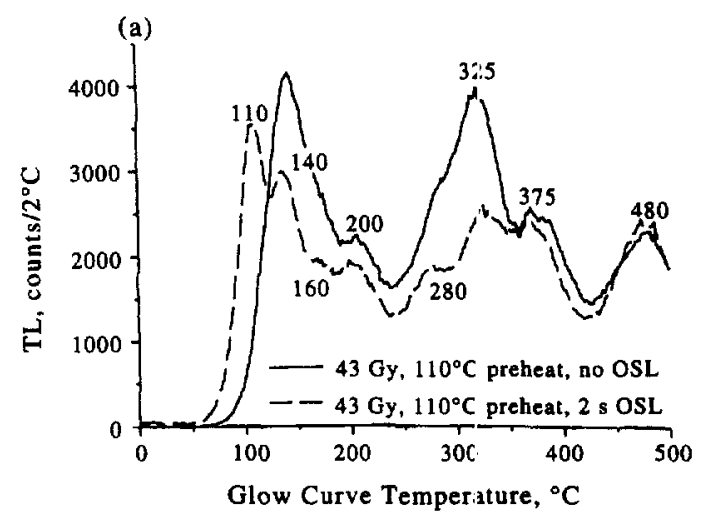

(b) Wavelength (nni)

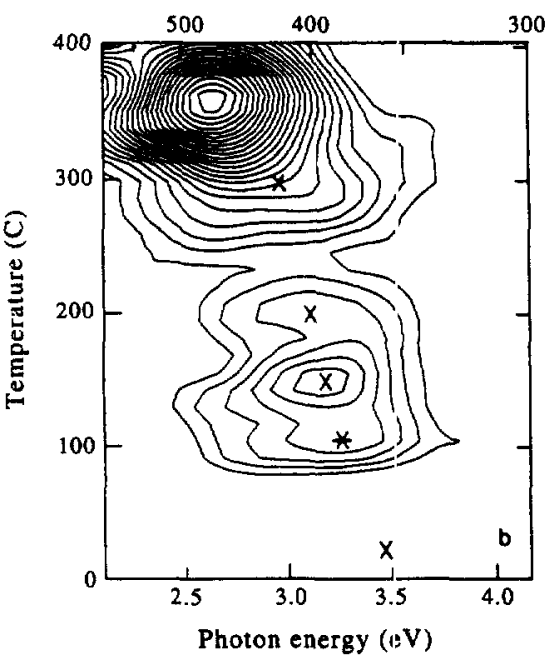

Fig. 6. (a) TL of Australian quartz W'IDG8 after bleaching and irradiating with $43 \mathrm{~Gy}$, and then being given a preheat to $110^{\circ} \mathrm{C}$, with one sample being exposed to green light for $2 \mathrm{~s}$; the peaks at 160 and $3.15^{\circ} \mathrm{C}$ bleach rapidly compared to the other peaks (from Wintle and Murray, 1997). (b) Spectral results for Australian quartz from Puritjarra (from Franklin et al., 1995) showing the TL peaks at $\sim 325, \sim 160$ and $110^{\circ} \mathrm{C}$, each with emission peaks which are probably related to the same luminescence centre, $\mathrm{X}$ at $22^{\circ} \mathrm{C}$ for OSL from another quartz, as in Fig. 7(a).

are underestimated by about $9 \%$, for $150 \mathrm{ka}$ by $15 \%$ and for $200 \mathrm{ka}$ by $22 \%$. For these samples the correction to the ages varies little with dose rate (from 1.5 to $5 \mathrm{~Gy} / \mathrm{ka}$ ). For samples over $250 \mathrm{ka}$ the natural dose rate plays an important role in defining the natural saturation level, and results in the definition of a practical dating limit which is dose rate dependent. For alkali feldspars of Scandinavian origin (with $11-13 \% \mathrm{~K}$ ), Mejdahl (1989) suggested that corrected ages can be obtained for samples as old as $400 \mathrm{ka}$; Balescu et al. (1997) suggested that for their lower K content feldspars $(7-8 \% \mathrm{~K})$ from north-western France, the upper age limit (after correction) may be as much as $800 \mathrm{ka}$ (see Section 8.3).

However, thermal decay following predicted kinetic behaviour is not the only cause of loss of lumi- nescence. Anomalous fading of the TL signal from feldspars of volcanic origin has been found (Wintle, 1973). It is also reported in some sedimentary feldspar separates, although it does not seem to occur to the same extent. This may be due to the TL signal being from a mixture of feldspar grains, only some of which exhibit anomalous fading. Mechanisms of anomalous fading have been discussed by Duller (1997) (section 2.3). After several years' study of anomalous fading (Visocekas, 1985) and the related phenomenon of an "afterglow", which is observed at low temperatures following irradiation at room temperature (Visocekas, 1993), a new possibility for dating volcanic feldspars has emerged. Zink and Visocekas (1996) have suggested using a near-infrared emission band centred around $700-710 \mathrm{~nm}$ which appears to be stable over hundreds of thousands of years. TL observed in this wavelength region increases with dose and also appears to bleach in a few hours, similar to its blue counterpart which shows anomalous fading. Unfortunately it is difficult to separate the TL emission at this wavelength from the black body radiation, thus making observation of the TL above $300^{\circ} \mathrm{C}$ difficult. However, very promising preliminary results have been reported for sanidines of volcanic origin, for which there are independent age estimates (Zink and Visocekas, 1997).

A peak at $150^{\circ} \mathrm{C}$ is found in laboratory-irradiated potassium feldspars. The electron trap responsible for this peak has been shown to compete with the deeper more stable traps, with the $330^{\circ} \mathrm{C}$ TL peak growing more slowly if laboratory irradiation is carried out at $130^{\circ} \mathrm{C}$, thus keeping the shallow traps empty (Mejdahl et al., 1992). This has led to a further correction procedure being applied to samples of over $15000 \mathrm{y}$ from West Greenland; this involves the value of $D_{\mathrm{E}}$ obtained for $25^{\circ} \mathrm{C}$ irradiations being multiplied by 1.35 . This effect needs further investigation for TL, OSL and IRSL for a much wider range of feldspars (Mejdahl and Christiansen, 1994).

\subsection{Feldspar $O S L$}

Feldspars were also the subject of the early OSL studies carried out with the $514.5 \mathrm{~nm}$ line from argon lasers (Huntley et al., 1985; Godfrey-Smith $e t$ al., 1988). The first emission spectra were obtained by combining measurements of stimulation using this line, whilst observing at wavelengths shorter than $500 \mathrm{~nm}$, and the $633 \mathrm{~nm}$ line from a krypton laser, whilst observing below $550 \mathrm{~nm}$ (Huntley et al., 1989). The spectra for the plagioclase feldspars were identical to the TL spectra of the same samples in this restricted wavelength region. Using narrow pass filters and the $633 \mathrm{~nm}$ stimulation, Jungner and Huntley (1991) demonstrated that the OSL is dominated by emission at $400 \mathrm{~nm}$ [Fig. 7(b)]. 
(a)

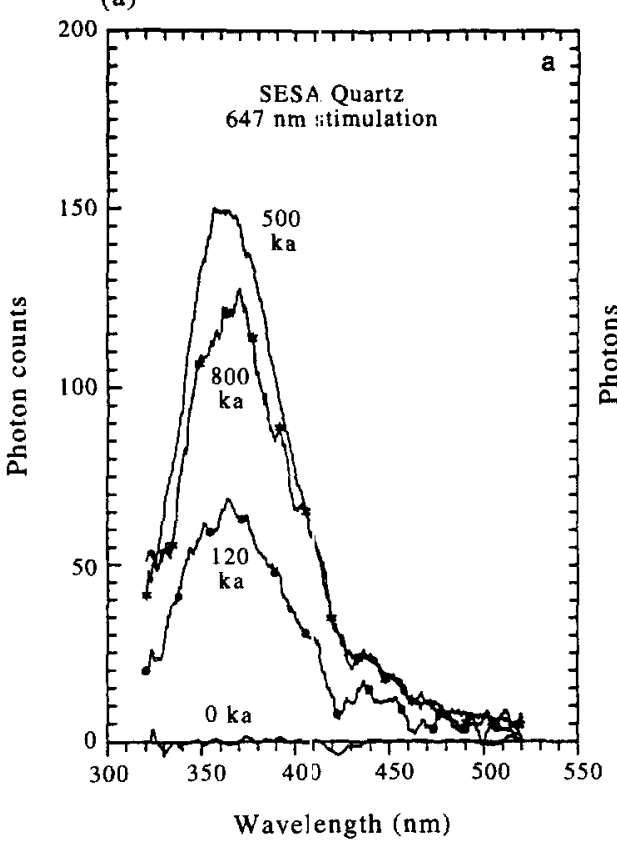

(b)

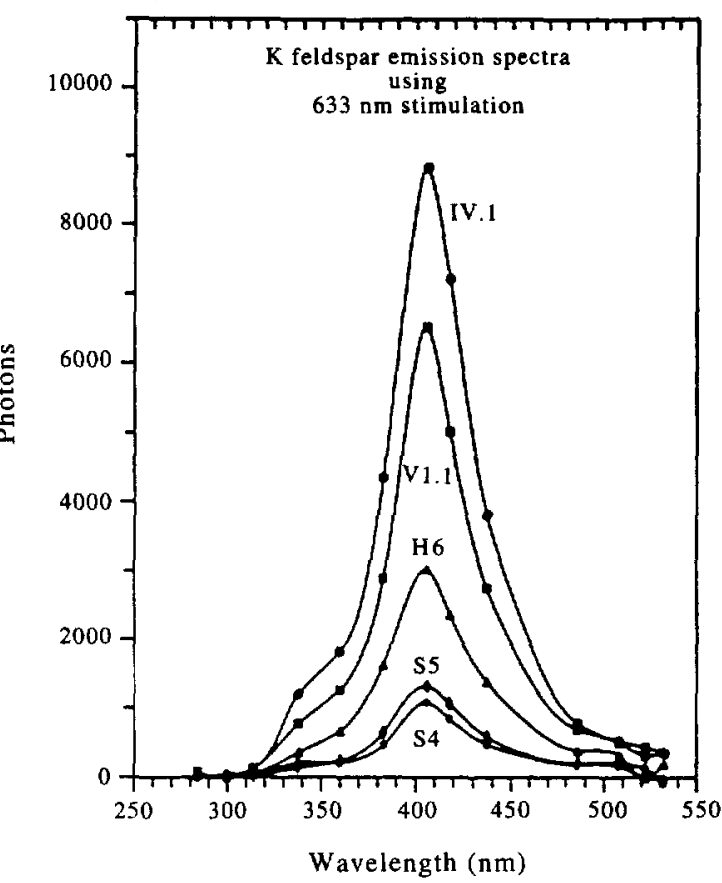

Fig. 7. (a) Emission spectra of several samples of sedimentary quartz from south-east South Australia aged from 0 to 800 la obtained for stimulation using the $647 \mathrm{~nm}$ line from a krypton laser (from Huntley et al., 1991). It differs from the $350^{\circ} \mathrm{C}$ TL spectrum of a similar sample in Fig. 2(a), but is similar to that of the $110^{\circ} \mathrm{C}$ TL peak as illustrated in Fig. 6(b). (b) Emission spectra of several samples of sedimentary $\mathrm{K}$ feldspars from Finland, aged about $100 \mathrm{ka}$, obtained for stimulation using the $633 \mathrm{~nm}$ linz from a helium-neon laser (from Jungner and Huntley, 1991).

Given the limited number of luminescence laboratories which have lasers available for optical stimulation (in Simon Fraser University and Oxford University), relatively few results have been obtained using the OSL (rather than IRSL) of feldspars since the initial studies were published in the 1980s. In fact the only dating application using green light stimulation of feldspar separates was in 1985 by Huntley et al. ir. their first paper. In an extensive study of the 4-1I $\mu \mathrm{m}$ and $43-54 \mu \mathrm{m}$ polymineral fractions from a sample of European loess ( $<20 \mathrm{ka}$ ), Questiaux (1991) used both $514.5 \mathrm{~nm}$ laser stimulation and $880 \mathrm{~nm}$ infrared diode stimulation, each with two set; of detection filters. No significant differences were found between any of the values of $D_{\mathrm{E}}$ obtained. Questiaux (1991) concluded that it would be appropriate to measure the larger grain size and that density separation could be applied, as carried out in an earlier TL study of older loess by Balescu et al. (1988).
Following the development of a filtered halogen lamp light source (Bøtter-Jensen and Duller, 1992), some fundamental studies have been reported in which the green light stimulated luminescence (GLSL) and IRSL signals of the same samples have been compared (Duller and Bøtter-Jensen, 1993; Li and Tso, 1997). Ever since infrared diodes were shown to give an easily measurable IRSL signal, optical dating of feldspars has been dominated by measurements made using IR stimulation. Even those laboratories with green light stimulation systems have used them almost entirely for quartz, whilst using IR stimuiation for feldspars. However, the different bleaching responses of the two signals as demonstrated by Duller and Bøtter-Jensen (1993) could be extremely useful for sediments where bleaching at deposition may have been inadequate to fully bleach either signal (see Section 8). This different bleaching behaviour suggests that these signals might be derived, at least in part, from two

Table 1. OSL trap parameters and lifetime at $20^{\circ} \mathrm{C}$

\begin{tabular}{|c|c|c|c|c|c|}
\hline$\%$ & $E(\mathrm{eV})$ & $\log _{10} S\left(S\right.$ in $\left.s^{-1}\right)$ & $\tau$ at $20^{\circ} \mathrm{C}(\mathrm{Ma})$ & Irrad & Reference \\
\hline 100 & $1.84 \pm 0.07$ & 15.3 & 600 & lab & Smith et al. (1990a) \\
\hline 20 & $1.39 \pm 0.07$ & 12.0 & 0.014 & nat & Huntley et al. (1996) \\
\hline 20 & $1.55 \pm 0.07$ & 12.9 & 2.0 & nat & \\
\hline 49 & $1.65 \pm 0.01$ & 13.43 & 28 & nat & \\
\hline 1 & $1.82 \pm 0.05$ & 14.1 & 4700 & nat & \\
\hline$>99$ & $1.88 \pm 0.03$ & 15.9 & 850 & nat & Wintle and Murray (in press) \\
\hline
\end{tabular}


separate traps. This hypothesis was backed up by the different thermal stabilities found for the GLSL and IRSL signals from the same feldspar separates taken from an alluvial sediment (L.i and Tso, 1997). They calculated that for the OSL signal, the lifetime at $10^{\circ} \mathrm{C}$ was about 1 million years, more than two orders of magnitude greater than that for the IRSL signal.

OSL has also been obtained for a wide range of stimulation wavelengths. Bøtter-Jensen et al. (1994) used a monochromator and halogen light source to stimulate at wavelengths ranging from $380-1020 \mathrm{~nm}$ (see Fig. 20 of Duller, 1997). The results obtained for a suite of well-characterised museum specimens of feldspar were used to try and link the spectral changes with the known crystal structure (Poolton et al., 1996).

\subsection{Feldspar IRSL}

Following the first announcement of the unexpected stimulation peak in the near infrared for a number of sedimentary feldspars (Hütt et al., 1988), considerable effort has been put into characterising the stimulation spectrum for a number of chemically-characterised museum specimens.

In particular, Bailiff (1993) and Bailiff and Barnett (1994) used a tunable titanium-sapphire laser to measure the stimulation spectra in the 700$1000 \mathrm{~nm}$ region of the near infrared. A chip of orthoclase $(8.4 \% \mathrm{~K})$ was found to have a stimulation spectrum which could be characterised by a Gaussian when plotted as a furction of photon energy (see Fig. 18 of Bøtter-Jensen, 1997). For this particular feldspar sample they showed that the stimulation peak at room temperature was at $854 \mathrm{~nm}(1.455 \pm 0.002 \mathrm{eV})$. These studies have been extended to a wider range of well-characterised feldspars, and to fine grain sediment samples from intertidal sediments of Holocene age (Barnett and Bailiff, 1997). The fine grain sediment samples were shown to contain both plagioclase feldspar and the potassium feldspar orthoclase. These studies showed that all samples not only had a stimulation peak close to $1.45 \mathrm{eV}$, but also a second, weaker, peak centred at about $1.6 \mathrm{eV}(\sim 775 \mathrm{~nm})$. The authors noted that there was a trend for the ratio of the intensity stimulated by the $\sim 1.6 \mathrm{eV}$ radiation to that by the $\sim 1.45 \mathrm{eV}$ radiation to be greater for potassium-rich feldspars than the sodium-rich feldspars, but found no systematic change in the position of the lower energy peak, as previously reported by Godfrey-Smith and Cada (1996).

Such studies aid the selection of the most appropriate diodes for more routine measurements. Commonly used diodes have emission peaks at $880 \mathrm{~nm}$ (e.g. TEMT 484) or $875 \mathrm{~nm}$ (Telefunken TSHA 6203), both of which have a width of about $80 \mathrm{~nm}$. Godfrey-Smith and Cada (1996) suggested that it might be more appropriate to use a small semiconductor laser tuned to $845 \mathrm{~nm}$.

Given that the stimulation wavelength for IRSL is well away from the visible emission region, it has been relatively easy to determine the emission spectrum. The first results were obtained by Huntley et al. (1991) for the $\mathrm{K}$ feldspars whose TL spectra had already been studied. They used IR diodes (775$980 \mathrm{~nm}$ ) for stimulation and found the emission peak to be at $410 \mathrm{~nm}$ (Fig. 8), with evidence of a less intense peak between 300 and $350 \mathrm{~nm}$. Measurements made on the plagioclase feldspars indicated that they emitted primarily at $570 \mathrm{~nm}$. Thus IRSL signals from both types of feldspar are passed by the Schott BG-39 filter (Fig. 6 of BøtterJensen, 1997), although the orange $570 \mathrm{~nm}$ emission will be less efficiently detected as the quantum efficiency of a bialkali photomultiplier tube starts to drop off in this wavelength region (Fig. 1 of BetterJensen, 1997). Using a CCD camera-based spectrometer, Krbetschek et al. (1996) observed emission bands at 330,410 and $560 \mathrm{~nm}$ for the natural IRSL of potassium-rich feldspars extracted from a sediment. They also reported the presence of emission bands at about 280 and $700 \mathrm{~nm}$, although these could not be detected directly because of the need to use a Schott BG-39 filter to prevent light from the stimulation source reaching the detector.

Rendell et al. (1995) used a spectrometer with two imaging photon detectors (IPDs) to give an extended spectral range $(200-800 \mathrm{~nm})$ (Fig. 17 of Bøtter-Jensen, 1997) (Luff and Townsend, 1993). They observed the emission peak at $290 \mathrm{~nm}$ for natural TL confirming the spectral results of Prescott and Fox (1993) and Prescott et al. (1994). They also found a $290 \mathrm{~nm}$ emission for IRSL from the same laboratory-irradiated sample, but no natural IRSL at this wavelength. This behaviour was

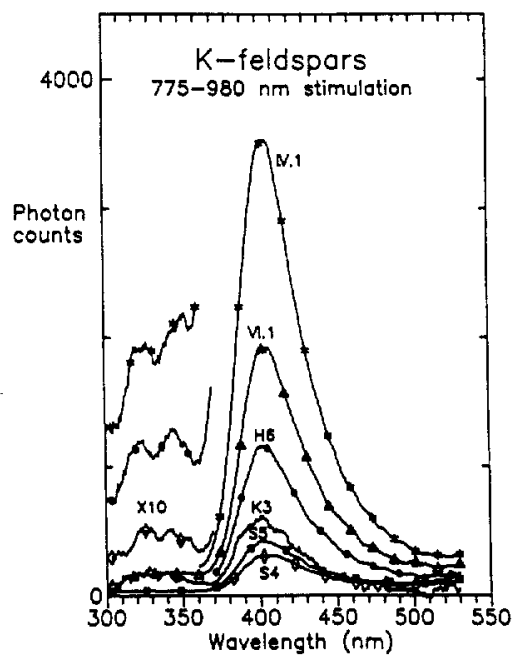

Fig. 8. IRSL emission spectra in the range $300-530 \mathrm{~nm}$ for K-rich feldspar obtained using IR diode stimulation (from Huntley et al., 1991). 
subsequently explained in a further study using the same system. Clarke and Rendell (1997a) found that the $290 \mathrm{~nm}$ IRSL emission from a laboratoryirradiated chip of albite could be removed by heating. As the preheat temperalure was increased up to $200^{\circ} \mathrm{C}$, there was also an inivial increase in the emission at $380 \mathrm{~nm}$, which subsequently decreased as $200^{\circ} \mathrm{C}$ was reached. Clarke and Rendell (1997b) demonstrated that the UV emission is probably related to a defect site associated with a sodium feldspar lattice structure. Sme.ll amounts of sodium feldspar may be found in some potassium-rich feldspars, and this explains their observation of $290 \mathrm{~nm}$ emission in all feldspar samples, whether museum specimens, feldspar standards or potassium-rich feldspars separated from secliments.

Experiments carried out involving a $220^{\circ} \mathrm{C}$ preheat show that sustained preheats, of $1 \mathrm{~min}$ or more, remove the $290 \mathrm{~nm}$ emission and simultaneously result in stabilisation of the emission at 380 and $560 \mathrm{~nm}$ (Fig. 9). This phenomenon results in very different behaviour of the IRSL signal when it is observed with each of the two commonly used filter combinations, namely the Corning 7-59 plus Schott BG-39 (as used in OSL/TL readers equipped only with infrared diodes) and the Hoya U-340 (as used in readers equipped with a green light stimulation system). The IRSL signal observed with the U-340 decreases with increasing preheat temperature, whereas it increases when observed with the other filter combination (Clarke and Rendell, 1997a). A preheat of at least $1 \mathrm{~min}$ at $220^{\circ} \mathrm{C}$ would eliminate differences between measurements made with different filters during a dating procedure.

Another effect of the observed phenomenon relates to the use of IR stimulation as a check for the purity of quartz (Section 1.2). If the check for IRSL was made with the U-340 filter, as might be the case in an OSL quartz dating procedure, very little signal would be detected from the natural samples. It would thus be preferable to make the IRSL test using either a BG-39 filter alone (or combined with a 7-59 filter) or an irradiated, but unpreheated, sub-sample.

There is limited information on the long-term thermal stability of the IRSL signal from feldspars. Anomalous fading of the IRSL signals from a large number of feldspars has been reported by Spooner (1992, 1994a). A few isothermal decay studies of IRSL from feldspars not exhibiting anomalous fading are reported in the literature ( $\mathrm{Li}$ and Wintle, 1992), but none have led to the derivation of trap depth values and calculated half-lives. Instead the trap depths and frequency factors have been determined using procedures analogous to those for TL analyses. Short and Tso (1994) proposed a method of analysis which used a non-linear heating rate. A simpler method was proposed by Li et al. (1997) which involves pulse annealing of samples after an initial preheat at $220^{\circ} \mathrm{C}$ for $10 \mathrm{~min}$ to isolate the more thermally stable part of the IRSL signal. Preliminary results obtained by Tso et al. (1996) on one K-rich and one Na-rich feldspar separate gave a lifetime of $\sim 10^{9} \mathrm{y}$ for the former, but one five

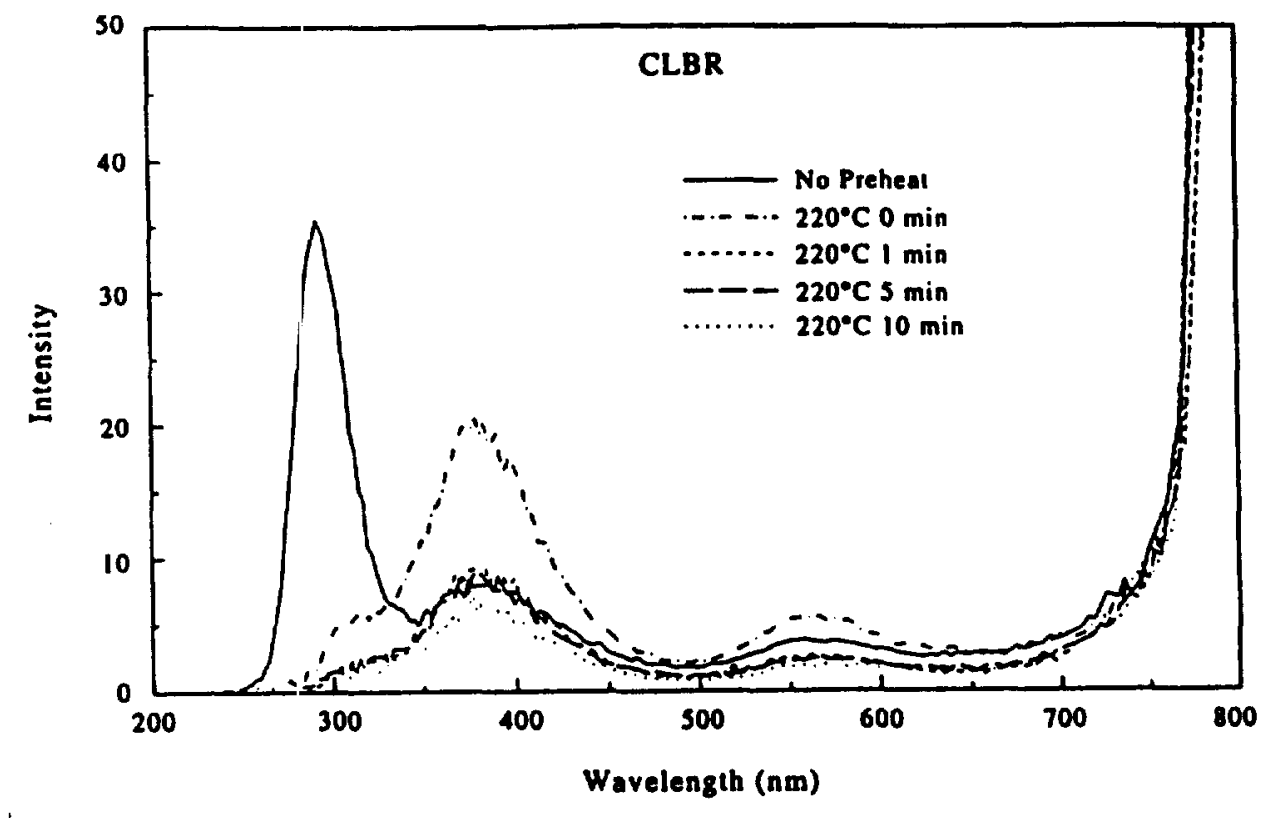

Fig. 9. IRSL emission spectrum over the range $200-800 \mathrm{~nm}$ obtained for a laboratory-irradiated chip of the sodium-rich feldspar albite (CLBR). Sustained heating for more than $1 \mathrm{~min}$ at $220^{\circ} \mathrm{C}$ removes all emission at $290 \mathrm{~nm}$ (from Clarke and Rendell, 1997b). 
orders of magnitude less for the latter. Further studies of this type are required on a wide range of feldspars. In a second study, Li and Tso (1997) carried out the same procedure on another K-rich feldspar separate and found a similar lifetime for the IRSL, but a lifetime two orders of magnitude less when the same procedure was used for the OSL.

\subsection{Calcite $T L$ and $O S L$}

Calcite, a crystalline form of $\mathrm{CaCO}_{3}$ found in nature as flowstone in caves, has a TL signal, with emission around $570 \mathrm{~nm}$. The potential for using at least one of the TL peaks for dating has been explored (Wintle, 1978; Debenham, 1983). However, applications have been few due to the limited nature of the application to cave deposits. Also, disequilibrium of the uranium decay chain needs to be accounted for, and the measurements required are themselves the basis of a better established dating method.

Ugumori and Ikeya (1980) presented the results of optical stimulation experiments on calcite from an archaeological site. Unlike the OSL measurements on feldspars and quartz, they used stimulation wavelengths shorter than the detection wavelengths. This work was not followed up, but the relatively high luminescence sensitivity of pure crystalline calcite may lead to rew possibilities using optical stimulation. For example, Liritzis (1994) has used light-sensitive TL signals for dating the construction of buildings made of limestone blocks; a more detailed study o: the bleaching characteristics of the TL from a number of limestone and marble samples has shown charge transfer from the 280 and $350^{\circ} \mathrm{C}$ TL peaks to a PTTL peak at around $180^{\circ} \mathrm{C}$ (Liritzis et al., 1996). OSL measurements would be more app-opriate for this type of dating application.

\subsection{Zircon $T L$ and $O S L$}

Zircon grains have long been realised to be useful dosimeters. Zircon grains have high concentrations of uranium in their lattice structure, and thus their dose rate is dominated by their self dose. This means that they are negligibly affected by changes in the dose rate of their environment, such as is caused by changing water content. Their dose rate is constant, as the uranium was incorporated in the lattice far back in geological time. TL dating of individual grains has been carried out (Templer and Smith, 1988) on zircons extracted from pottery and TL emission spectra of zircons have been obtained by Huntley et al. (1988a). However, a grain-bygrain approach is required because each grain has a different uranium content and a different TL sensitivity. A significant improvement ir. measurement of zircon grains was achieved by Smith et al. (1991), who used an imaging photon detector (IPD) instead of a photomultiplier tube for measuring the TL signal from a number of grains simultaneously.

An additional problem is caused by the original formation of the zircon grain, a process which results in a non-uniform crystal structure (Templer, 1985, 1986). This meant the simple comparison of the natural $\mathrm{TL}$ and the regenerated signal from the same grain may not result in an equivalent dose which can be combined directly with measurements of the grain's internal dose rate based on the average uranium content.

The same problem of sample inhomogeneity applies to the OSL signal that is obtained from zircon grains. Zircon grains are known to produce OSL signals (Smith, 1988), and their contribution to the OSL signal from quartz grains separated from a sediment needs to be considered if heavy liquid separation has not been employed to remove heavy minerals, including zircon grains.

\section{OPTICAL BLEACHING}

\subsection{Effects of light exposure}

It was recognised early on in the development of TL dating of pottery that samples should be prepared in reduced light levels, ambient lighting being found to decrease the TL signals. The role of sunlight as a bleaching agent which could reduce TL signals to a finite but stable level was not exploited until results were reported for fine grain silicate minerals extracted from an ocean core (Wintle and Huntley, 1979). Dating of sediments had been reported in the Russian scientific literature several years earlier than this, but the mechanism which was thought to reduce the TL signal was the break-

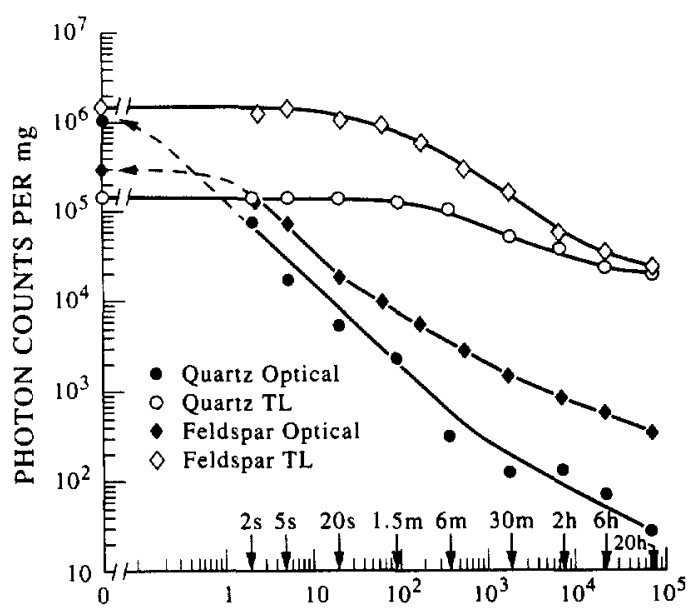

DURATION OF SUNLIGHT EXPOSURE (seconds)

Fig. 10. Reduction of natural OSL and TL signals from samples of quartz and feldspar when exposed to sunlight. The OSL signals are reduced both more rapidly and more completely than the TL from the same sample (from Godfrey-Smith et al., 1988). 
down and grinding of bedrock by ice at the base of the massive ice sheets which covered northern parts of Europe during glacial psriods.

The much greater speed of reduction of OSL than TL signals was demonstrated for quartz and feldspar by Godfrey-Smith et al. (1988). Figure 10 shows the reduction in quartz and feldspar OSL as a function of sunlight, with the quartz OSL (as measured with an argon ion laser) being reduced to $\sim 1 \%$ of the initial value by 20 s exposure to direct bright sunlight. The feldspar OSL bleached less rapidly under the same conditions, taking about $6 \mathrm{~min}$ to reach that level of reduction. Weak OSL signals could still be observed after several hours' sunlight exposure, but in the case of quartz were less than $0.01 \%$ of the initial value and close to the equipment background. In the same time the quartz TL signal measured at $375^{\circ} \mathrm{C}$ had only reduced to $10 \%$ of its initial value and appeared to be reaching a finite residual level. The feldspar TL shows that a greater reduction is possible on extended light exposure. These findings are consistent with finite TL signals being found for quartz (and feldspar) from modern sediments, but with zero OSL signals (Godfrey-Smith et al., 1938; Huntley et al., 1985). Figure 11 shows the sunlight bleaching of the TL and IRSL of sedimentary feldspar grains, again indicating the more rapid and more complete bleaching of the optically stimulated signal, in this case the IRSL

3.1.1. Untrapping of electrons. The most obvious explanation for the decay in the luminescence signals when they are exposed to light is that the OSL is produced by the recombination of electrons which have been optically detrapped, and the observed decay is due to the depletion of the trap with continual stimulation. For quartz, release of charge (presumed to be electrons) from these traps can also be monitored by the observation of a $T L$ peak at $110^{\circ} \mathrm{C}$ after light exposure. This phototransferred TL (PTTL) peak has been shown to vary in phase with the OSL signal and with the loss

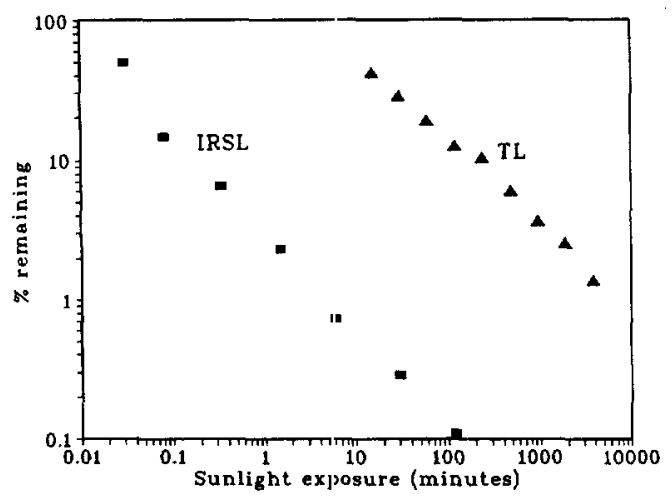

Fig. 11. Reduction in natural IRSL and TL on exposure to bright sunlight for K-rich feldspars separated from sand. of TL from the light-sensitive $325^{\circ} \mathrm{C} \mathrm{TL}$ peak (Stoneham and Stokes, 1991; Murray, 1996b; Wintle and Murray, 1997). The initial OSL signal from quartz had already been related to the $325^{\circ} \mathrm{C}$ TL peak (Smith et al., 1986; Kaylor et al., 1995).

3.1.2. Reduction in the number of luminescence centres. Whilst untrapping of electrons is the most likely explanation for the decay of the OSL signals from quartz under prolonged stimulation, the reduction in the number of luminescence centres available for radiative recombination during luminescence measurements subsequent to bleaching must also be considered (McKeever, 1991, 1994). Computer simulations by McKeever and Morris (1994) have shown that such a reduction in luminescence centres, linked to the availability of at least one non-radiative centre, could explain the decay of some luminescence signals from quartz which were observed experimentally (Morris and McKeever, 1994). Allocation of the effect of bleaching on other luminescence signals, such as the PTTL peak at $110^{\circ} \mathrm{C}$, to either loss of trapped electrons or loss of luminescence signals is less clear-cut (Wintle and Murray, 1997). No similar study has been done on any feldspar, although a characteristic feature of their behaviour is that there is either no loss in TL at any temperature after the IRSL has been removed by the measurement (Musson et al., 1994) or there is a uniform but limited reduction in TL (e.g. $\sim 10 \%$, Musson and Wintle (1994) or $\sim 20 \%$, Richardson et al. (1997)).

\subsection{Use of different wavelengths}

Comprehensive bleaching studies using wavelength bands selected from a tungsten halogen lamp with narrow pass filters have been carried out on quartz. The bleaching behaviour of the 325 and $375^{\circ} \mathrm{C}$ TL peaks as a function of wavelength was reported by Spooner et al. (1988). Figure 12 shows the power required to reduce these peaks (given here as $320^{\circ} \mathrm{C}$ and $370^{\circ} \mathrm{C}$ ) to $0 \%$ and $80 \%$ of their initial values, respectively. Also shown is the power required to reduce the OSL signal obtained using the $514.5 \mathrm{~nm}$ argon laser line by 5, 50 and $95 \%$, respectively (Spooner, 1994b). It can be seen that it is extremely difficult to reduce the $370^{\circ} \mathrm{C}$ TL peak (and a higher peak at $480^{\circ} \mathrm{C}$, see Fig. 5(a)) with wavelengths greater than $400 \mathrm{~nm}$. Bleaching of these peaks appears to be restricted to the violet part of the visible spectrum and the near ultraviolet. The $325^{\circ} \mathrm{C}$ peak also shows a similar relationship for the power levels required at different wavelengths from 320 to $400 \mathrm{~nm}$, but with 100 times less power being required to achieve the same percentage reduction.

In addition, significant reduction was also observed for wavelengths up to $630 \mathrm{~nm}$, albeit for increased power levels. The variation in power with wavelength from 400 to $600 \mathrm{~nm}$ was similar for the 


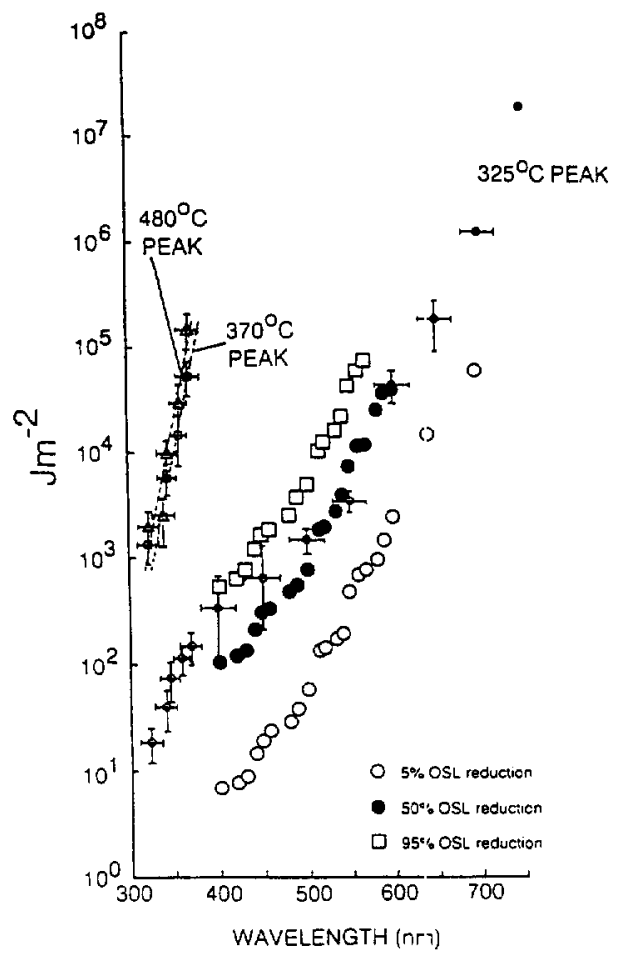

Fig. 12. The energy required, as a function of wavelength, to reduce the 370 and $480^{\circ} \mathrm{C}$ TL peaks of quartz to $80 \%$ of their initial intensity, to bleach completely the $325^{\circ} \mathrm{C}$ TL peak, and to produce 5,50 or $9: 5 \%$ reduction in the OSL stimulated using the $514.5 \mathrm{~nm}$ laser line (from Spooner, 1994b).

$325^{\circ} \mathrm{C}$ peak and the OSL signal, again demonstrating their intimate relationship. Close comparison of these results with the transmission characteristics of the filters in the stimulation filter pack of the Risø green light system (Fig. 8 of Better-Jensen, 1997) indicates that exposure of irradiated quartz to the green light would not affect the $370^{\circ} \mathrm{C}$ TL peak, but would cause rapid reduction in the $325^{\circ} \mathrm{C}$ TL peak and the OSL signal. Since there is no reduction in the 370 and $480^{\circ} \mathrm{C}$ peaks in this wavelength region, it is unlikely that the electron traps for these peaks contribute directly to the OSL signal, although Murray and Wintle (1997) have shown that there is a contribution of $\sim 1 \%$ from traps in this temperature region. The inclusion of the more energetic wavelengths below $500 \mathrm{~nm}$ in this stimulation source must be borne in mind when experimental results obtained with this source are compared with those from the coherent, monochromatic light from the $514.5 \mathrm{~nm}$ argon ion laser line; as noted earlier, Murray and Wintle (1997) ccncluded that the broad band green light in the Risø OSL/TL reader acts as a light source with a single wavelength of $468 \mathrm{~nm}$. Duller and Botter-Jensen (1996) have made a direct comparison of the behaviour of the OSL signal obtained using the broad band green light $(420-560 \mathrm{~nm})$ and narrow wavebands with $25 \mathrm{~nm}$ resolution from 420 to $630 \mathrm{~nm}$ obtained using a narrow band pass monochromator in front of the stimulation light source. They concluded that, once a correction had been applied for the variation in stimulation efficiency as a function of wavelength, the shapes of the OSL decay curves were the same over the range from 425 to $575 \mathrm{~nm}$. This suggests that the traps and luminescence centres used in the production of OSL are the same, irrespective of wavelength.

\subsection{Use of different power}

Relatively few OSL experiments have been reported in which the power level is varied, whilst retaining the same stimulation wavelengths. The photon flux density at the sample is important as it has a bearing on the mechanism of charge untrapping, whether the process uses one or more photons. Using short stimulation times with the $514 \mathrm{~nm}$ laser line, Spooner (1994b) showed that the OSL intensity of a quartz sample was linear with the power of the laser beam over three orders of magnitude $\left(0.28-238 \mathrm{~mW} \mathrm{~cm}^{-2}\right)$. He concluded that this behaviour was indicative of a single photon absorption process, as originally proposed by Hütt $e t$ al. (1988).

The power is also likely to affect the rate of charge untrapping, particularly if shallow traps are involved in the production of the OSL. This could alter the shape of the OSL decay curve, and this led Duller and Bøtter-Jensen (1996) to measure full OSL decay curves for quartz illuminated with green light from a broad band $(420-560 \mathrm{~nm})$ light source. The decay curves obtained over a power range covering two orders of magnitude $\left(0.11-12 \mathrm{~mW} \mathrm{~cm}^{-2}\right)$ were almost identical when plotted with the OSL given in counts per $\mathrm{mJ}$ (rather than in cts/s).

For dating studies, power levels are usually selected to be as high as possible, in order to obtain the maximum luminescence signal output. It is lack of available power that is the reason for green light emitting diodes not being adopted as a cheap and robust alternative to the filtered green light (Galloway, 1993, 1994)

\subsection{Natural bleaching spectrum}

In the natural environment, bleaching of the TL and OSL signals occurs as the result of exposure to all solar wavelengths that reach the Earth's surface. Atmospheric absorption bands place a lower limit on the wavelengths available in the near UV. A typical mid-day solar spectrum is shown in Fig. 13(a) (from Baray and Zöller, 1994). This gives the range of wavelengths experienced by grains in an aeolian sediment.

Grains which are transported underwater, never having been exposed to direct sunlight, will have experienced a more restricted spectrum due to absorb- 


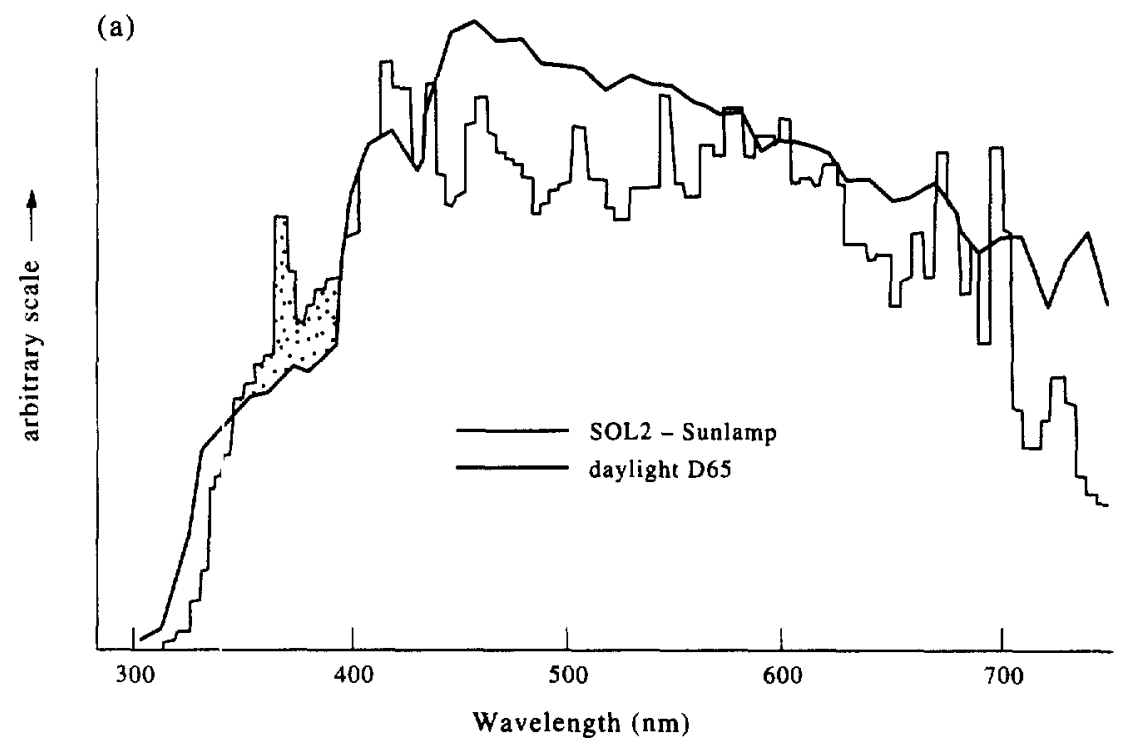

(b)

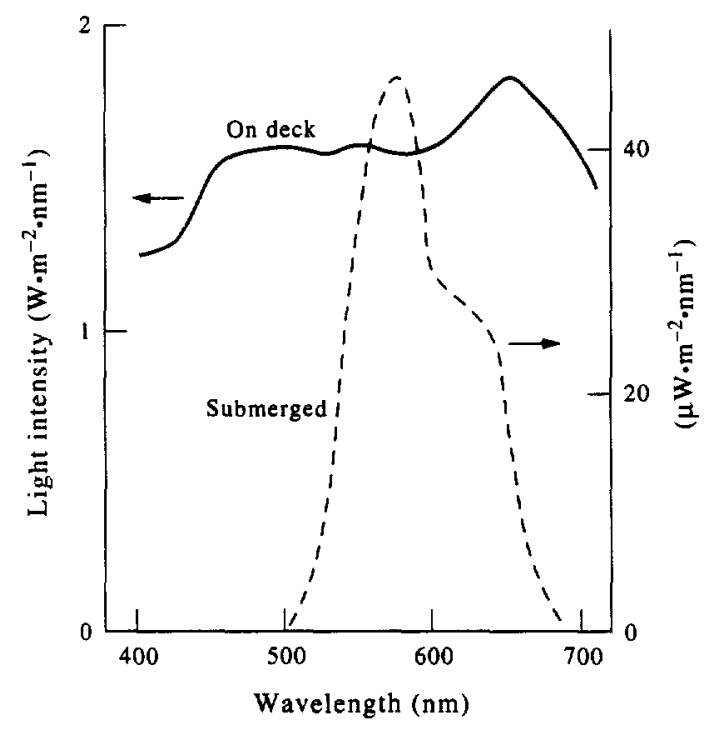

Fig. 13. (a) Spectra lor SOL2 sunlamp and a daylight standard (from Baray and Zöller, 1994). (b) Measured natural solar spectra obtained on deck and at a depth of $4 \mathrm{~m}$ in a turbid river (original from Berger and Luternauer, 1987). Note spectral attenuation below $500 \mathrm{~nm}$ and above $690 \mathrm{~nm}$ (from Fuller et al., 1994).

tion and scattering in the water column (Berger, 1990). Any sediment in the water will restrict the spectrum even further, ais shown by Berger and Luternauer (1987) in their study of the TL of fine grained sediment from the Fraser River [Fig. 13(b)]. Underwater bleaching experiments on the TL and OSL signals from quartz and TL and IRSL signals from feldspars have been carried out at depths of up to $14 \mathrm{~m}$ below a calm sea surface (Rendell et al., 1994b). The IRSL and OSL signals were effectively zeroed by $3 \mathrm{~h}$ exposure at water depths down to about $10 \mathrm{~m}$, whereas the TL signals were reduced by about $50 \%$.

\subsection{Use of laboratory light sources for bleaching}

Since it was first established that the TL signal in modern sediments was not zero (Wintle and Huntley, 1979), it was realised that fixed intensity laboratory light sources would need to be used to attempt to establish the signal which would have been found in sample grains at deposition. Solar radiation in many parts of the world is not suffciently constant to be used for laboratory experiments, and lamps were chosen which had spectral characteristics similar to sunlight, e.g. the Hönle SOL2 lamp, whose spectrum is reproduced in Fig. 13(a). Comparison of these spectra with the 
data of Fig. 12 shows that the higher temperature peaks will be bleached by both sunlight and the SOL2. Baray and Zöller (1994) expressed concern at the effect of the peak in the spectrum of the SOL2 at about $370 \mathrm{~nm}$, shown shaded in Fig. 13(a). Berger (1995) has strongly advised against the use of any unfiltered sunlamp for laboratory bleaching experiments, in particular for the dating of fine grain sediments by TL. Also it should be noted that the SOL2 intensity is several time:s that of direct sunlight.

An alternative laboratory bleaching approach has been used for TL dating of feldspars, in which a range of residual signals is obtained using a fluorescent lamp (Philips TL05) (Mejdahl and Christiansen, 1994). This lamp has a significant ultraviolet component, but was chosen as it produced residual glow curves for $\mathrm{K}$ feldspars which were the same as those produced by natural sunlight.

\section{GROWTH CURVES}

A growth curve is the name given to the plot of luminescence signal versus dose. The signal may be $T L$ or OSL (including IRSL). For TL, the luminescence signal may be the TL at a paticular temperature $\left(\right.$ e.g. $\left.375^{\circ} \mathrm{C}\right)$ or an integrated TL signal over a particular temperature range, provided that the signals show sufficient thermal stability. For TL the luminescence signal is usually expressed in counts/ sec and the magnitude will depend upon the heating rate, as well as filters and collection geometry. The signals may also be shown normalised by weight when values are to be compared (e.g. cts/s/mg). The OSL may be expressed as the total number of counts, as obtained in the integration of the OSL decay curve as stimulation progresses, or as the initial count obtained in a fixed time, e.g. $0.4 \mathrm{~s}$.

\subsection{Response to radiation}

The radiation response is obtained by carrying out laboratory irradiations with a calibrated source, usually beta or gamma. In the simplest case the luminescence signal will increase with dose as electrons produced by ionisation are trapped at defects in the crystal lattice. A finite number of electron traps of a particular type (e.g. corresponding to the $325^{\circ} \mathrm{C}$ TL peak) exist. Thus, although the growth will appear to be linear at low dose, it is in fact in the form of a saturating exponential with $I=I_{\infty}\left(1-\mathrm{e}^{-D / D_{0}}\right)$ where $I$ is the intensity of luminescence corresponding to a dose $D, I_{\infty}$ is the maximum signal attainable and $D_{\mathrm{o}}$ is a parameter which characterises the curve. Given the number of different electron traps found for a single mineral type, and the extent to which they are widened by perturbation of the crystal lattice due to impurities and by the temperatures at which measurements are made (i.e. well above absolute zero), it is amazing that the luminescence signal derived from the group of grains making up the mineral separate (or from (a)

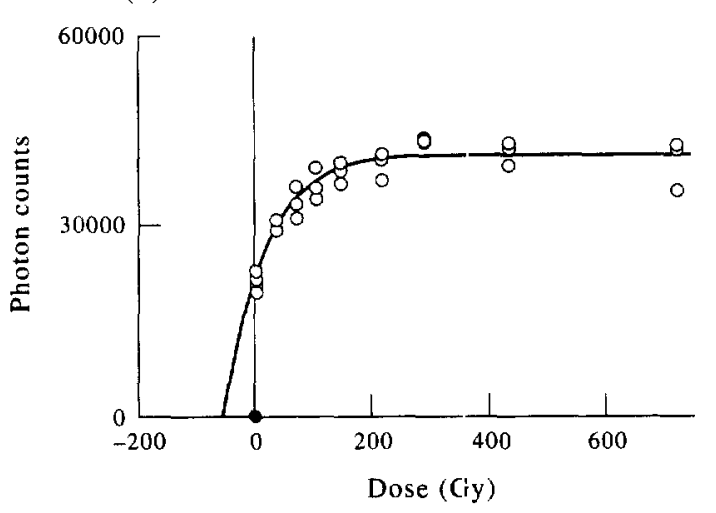

(b)

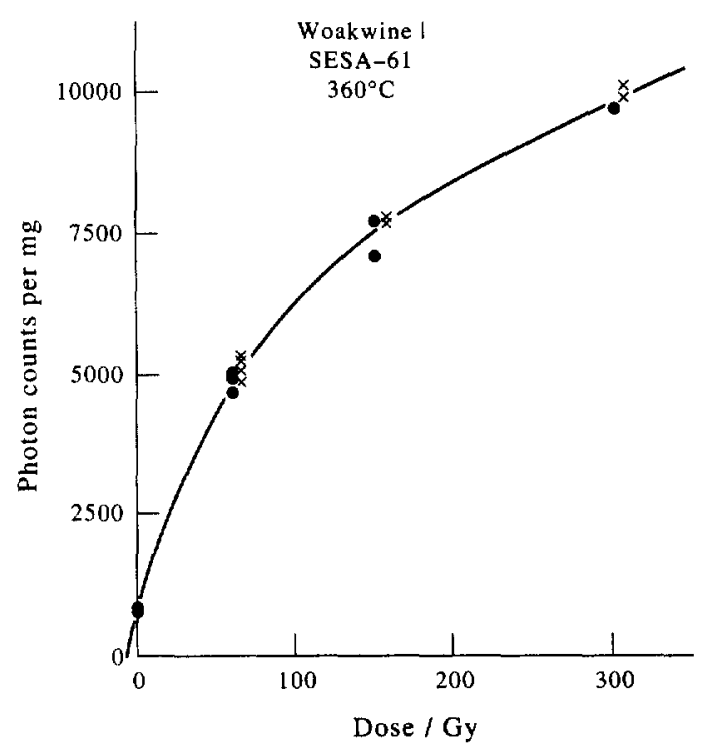

Fig. 14. (a) Growth curve of initial OSL signal from quartz from a $122 \mathrm{ka}$ dune sand (SESA-61) showing saturating response which would preclude the dating of similar, but older, samples (from Huntley $e t$ al., 1996). (b) Combined additive dose $(x)$ and regenerated (O) growth curves for $\mathrm{TL}$ at $360^{\circ} \mathrm{C}$ for quartz from the same dune. The data are fitted by an equation incorporating a saturating exponential and a linear signal. This is an example of the Australian slide method of equivalent dose determination (from Huntley et al., 1994). 
the fine grain fraction from a loess sample) shows exponential behaviour. Figure 14(a) shows the initial OSL signal as a function of added laboratory dose for quartz from a $120 \mathrm{ka}$ dune sand (Huntley et al., 1996).

\subsection{Non-linearity of response}

In spite of the general statement made above, there are some significant deviations from this behaviour for both young and old samples.

4.2.1. For very young samples. Early studies of pottery had shown that the early growth of TL with dose was often supra-linear, with a very low initial response to dose which resulted in growth curves for heated samples which did not pass through the origin (Flen.ing, 1970). By studying this response for samples whose ages were known independently, it was concluded that the growth of $\mathrm{TL}$ after burial followed the same pattern. Samples are dated by construction of two growth curves, one for doses added to the natural sample and one for doses after the natural sample has been heated to measure its TL, the so-called second glow growth curve. The dose intercept between the two straight line fits (ignoring the origin on the second glow growth curve) is taken as the radiation dose received by the grains since they were fired. In the worst cases, Fleming (197(1) found that, if supra-linearity was not measured and allowed for, TL ages for Romano-British pottery were underestimated by up to $50 \%$. Similar supra-linear behaviour has been reported for feldspars extracted from some young sediments (Lundqvist and Mejdahl, 1987).

4.2.2. Approach to saturation. As samples approach saturation, as shown for OSL in Fig. 14(a), the curve fitting procedure becomes difficult unless the saturation level $I_{\infty}$ can be well established. Application of 1erge laboratory doses to quartz from the same ciune sand SESA 61 has shown that the $375^{\circ} \mathrm{C}$ TL grows in a very different way [Fig. 14(b)]. A more extreme curve is shown in Fig. 18(a) for an older dune sand SESA 72, which will be discussed later. The growth appears to be best fitted by the combination of a saturating exponential and linear growth function. This combination appears to explain the behaviour of the peak when irradiated either in nature or in the laboratory. This contrasts with behaviour reported by Chawla et al. (1992) who found a saturation level above which growth could be achieved only by laboratory irradiation (Fig. 15).

\subsection{Natural versus artificial irradiation}

Since all luminescence dating applications require the estimation of the radiation exposure that has occurred since the zeroing event, it is necessary that the response observed in the laboratory is the same as that which pertained in the environment.

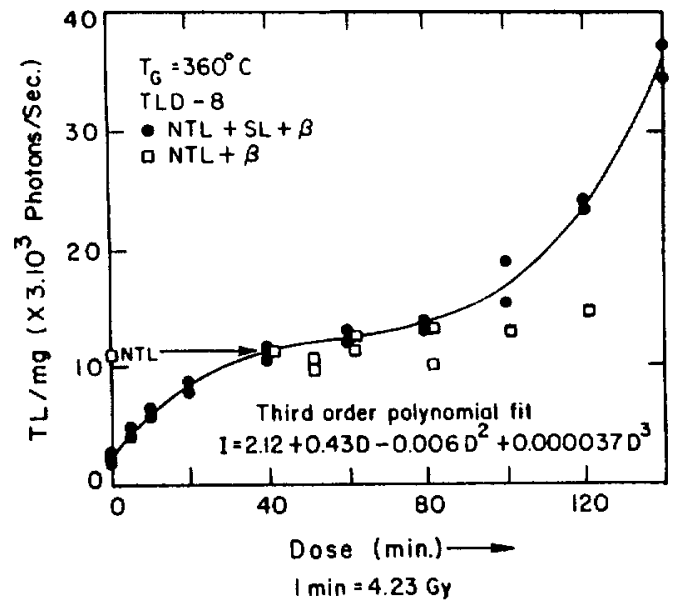

Fig. 15. Additive dose and regenerated growth curves for TL at $360^{\circ} \mathrm{C}$ for quartz from India. A polynomial fit was used for the regenerated data set, which show an increase in TL signal at higher doses (from Chawla et al., 1992).

However, there are several reasons why this may not be true.

Firstly, it is obvious that the dose rates used in the laboratory are many orders of magnitude higher than those experienced in nature. Experiments have been conducted using dose rates which vary over about 3 orders of magnitude (Valladas and Valladas, 1982), and there is some evidence of differences in response for the highest practicable laboratory dose rates.

Secondly, and related to the previous difference, is the effect of the total time period over which the dose is administered. In particular, during natural irradiation the shallow, thermally unstable traps, such as those giving rise to the $110^{\circ} \mathrm{C} \mathrm{TL}$ trap, are effectively empty. Although charge is able to be trapped, it will soon become thermally untrapped, whereas in laboratory irradiations at ambient temperature the shallow traps become filled and may even become saturated. Given the delivery of a fixed number of electrons to the conduction band by a fixed radiation dose, the competition between deep traps and shallow traps for electrons during irradiation may result in different sensitivities of the deep trap under the different irradiation conditions. Such behaviour has been demonstrated for $\mathrm{K}$ feldspars, in which the peak at $150^{\circ} \mathrm{C}$ competes for electrons. In experiments in which irradiations at $130^{\circ} \mathrm{C}$ were compared with irradiations at room temperature, the high-temperature $\mathrm{TL}$ peak at $330^{\circ} \mathrm{C}$ was found to grow at a different rate [Fig. 16(a)] (Mejdahl et al., 1992). This behaviour was reported for a number of sedimentary samples from Scandinavia which were thought to have a common origin, namely the Fennoscandian Shield, from which glacial action removed a considerable thickness of bedrock to form the sediments which were deposited as the ice retreated at the end of each major glaciation. For a set of sediments from 
(a)

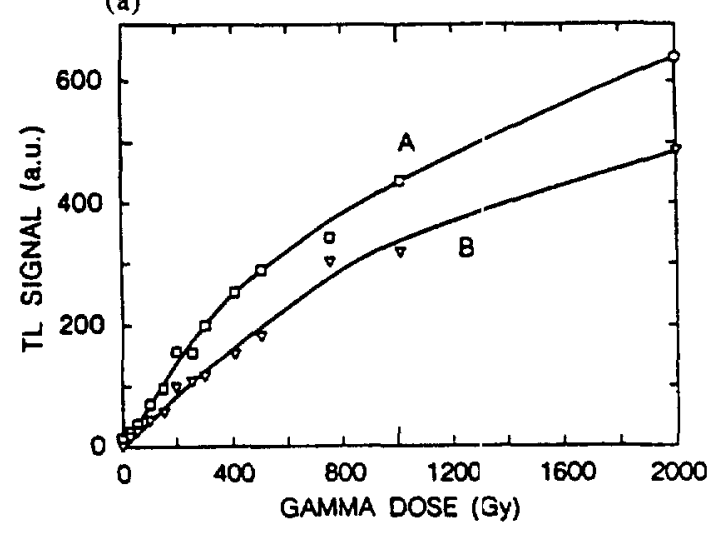

(b)

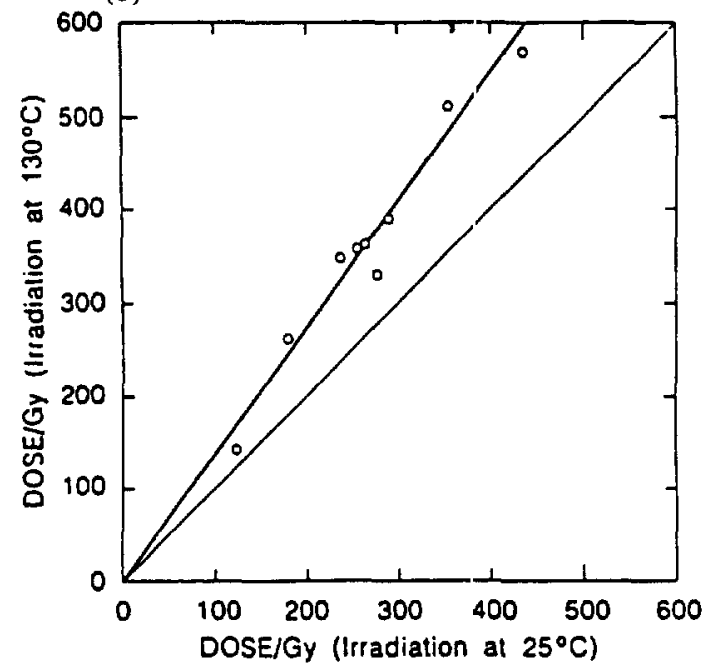

Fig. 16. (a) Regenerated growth curves for the $330^{\circ} \mathrm{C} \mathrm{TL}$ peak for K-rich feldspar from a Darish sediment. The sub-samples for curve A were irradiated at room temperature and those for curve $B$ at a temperature of $130^{\circ} \mathrm{C}$. (b) Doses obtained for sedimentary K-rick feldspar from the Thule area of Greenland for irradiations at $130^{\circ} \mathrm{C}$ and at room temperature, showing the former are about $35 \%$ larger (from Mejdahl et al., 1992).

Greenland, all values of $D_{\mathrm{E}}$ were found to be $35 \%$ larger when irradiation was performed at $130^{\circ} \mathrm{C}$, rather than at $25^{\circ} \mathrm{C}$ [Fig. 16 (b)]. A correction for the effect of this competitive behaviour is now made routinely for samples over 15000 years old in this region (Mejdahl and Christiansen, 1994). Unfortunately, it is not known if this effect is widespread, although published glow carves of feldspars often show a peak at about $150^{\circ} \mathrm{C}$ for irradiated samples. Further experiments at elevated temperature are needed to understand this phenomenon and to provide the data on which to base a laboratory protocol for dating. It is possible that laboratory irradiation units will need to be fitted with a sample heating stage for the clating of certain samples.
A third difference between natural and artificial irradiation, which also relates to the difference in time over which the irradiation is made, concerns the redistribution of charge which occurs as electrons are thermally ejected from shallow traps. In TL dating applications it is assumed that the transfer from shallow to deep traps is small, with the released electrons either undergoing radiative recombination or losing their energy as phonon interaction. More thought has been given to charge transfer in OSL studies, in which laboratory preheat procedures have been designed to remove charge from shallow light-sensitive traps which might also contribute to the OSL signal (Rhodes, 1988). During preheating charge may also be transferred from light-insensitive traps, which are thermally stable at ambient temperature, to the OSL trap(s). This was given as the cause of higher than expected OSL observed when zero-age sediments were preheated, and for which the OSL increased with preheat temperature (Godfrey-Smith et al., 1988).

A fourth reason for differences between the OSL signals following natural and laboratory irradiation can be ascribed to a sensitivity change caused by a change in the luminescence efficiency. Aitken and Smith (1988) reported an increase in OSL sensitivity as the preheat temperature was raised. They monitored the sensitivity changes using the OSL from a small test dose given after the preheat and showed that the most rapid change in sensitivity was observed between 220 and $280^{\circ} \mathrm{C}$, although sensitivity enhancement continued up to $600^{\circ} \mathrm{C}$. Following their suggestion that the increase in sensitivity of the $110^{\circ} \mathrm{C}$ TL peak followed a similar pattern, Stoneham and Stokes (1991) suggested the use of the $110^{\circ} \mathrm{C} \mathrm{TL}$ peak to monitor for sensitivity changes following preheating, initially with regard to multiple aliquot dating procedures. Stokes (1994) went further and suggested that the $110^{\circ} \mathrm{C}$ peak could be used to monitor for sensitivity changes in a single aliquot regeneration OSL protocol. Wintle and Murray (in press) have observed major differences between natural and laboratory-irradiated quartz sediments for the $110^{\circ} \mathrm{C}$ TL sensitivity response for preheats from $160^{\circ} \mathrm{C}$ to $280^{\circ} \mathrm{C}$. This difference was thought to be due to an enhancement in the naturally irradiated samples which occurs over the burial period.

\subsection{Quartz versus feldspar}

There have been several reports of feldspar TL signals being preferred for dating older samples. For example, fine grain quartz, extracted from European loess by etching with $\mathrm{H}_{2} \mathrm{SiF}_{6}$, shows nonlinearity of growth, and apparent saturation at dose values far lower than for the mixed mineral TL signal for the same sample (Wintle, 1982). The latter includes both the quartz and feldspar TL, although 
the relative proportions will vary with the choice of detection filters (Debenham, 1987). Figure 17 shows the natural TL glow curves from a fine-grain sediment sample which have been obtained using two different filters (a Schott UGI1 and a Corning 7-59 with an HA-3) and two sample treatments (normal preparation of fine grains and an additional treatment with $35 \%$ fluorosilicic acid for 3 days). It should be noted that all curves can be represented by two signals, designated $\mathrm{A}$ and $\mathrm{B}$, and although their relative proportions change, neither are eliminated either by choice of filter or by selective etching to remove the fine-grain feldspar. Debenham concluded that signal $B$, best observed with a blue filter, is of limited use for dating sediments because of its resistance to bleaching and its early saturation. However, it is not possible to observe type $A$ or type B signals alone. From Fig. 17 it can be seen that there is always some contribution from both signals in the region of the peaks of $A$ or $B$. However, it is possible to treat the TL curve mathematically and deconvolve the signals (Debenham, personal communication).

A small number of intercomparisons have been carried out for sand-sized grains, for example by Rendell et al. (1991, 1994a). In these examples the ages are compared, rather than the values of $D_{\mathrm{E}}$, since the internal dose rate of the potassium feldspars is higher. A particularly interesting comparison for older samples can be seen in Fig. 18, where the growth curve for the $375^{\circ} \mathrm{C}$ TL of quartz grains (Huntley et al., 1994) is compared with that for the IRSL of micro-inclusions from the same sample (Huntley et al., 1993a). The grains were separated from a beach sand deposited about $200 \mathrm{ka}$ ago during the penultimate interglacial and forming

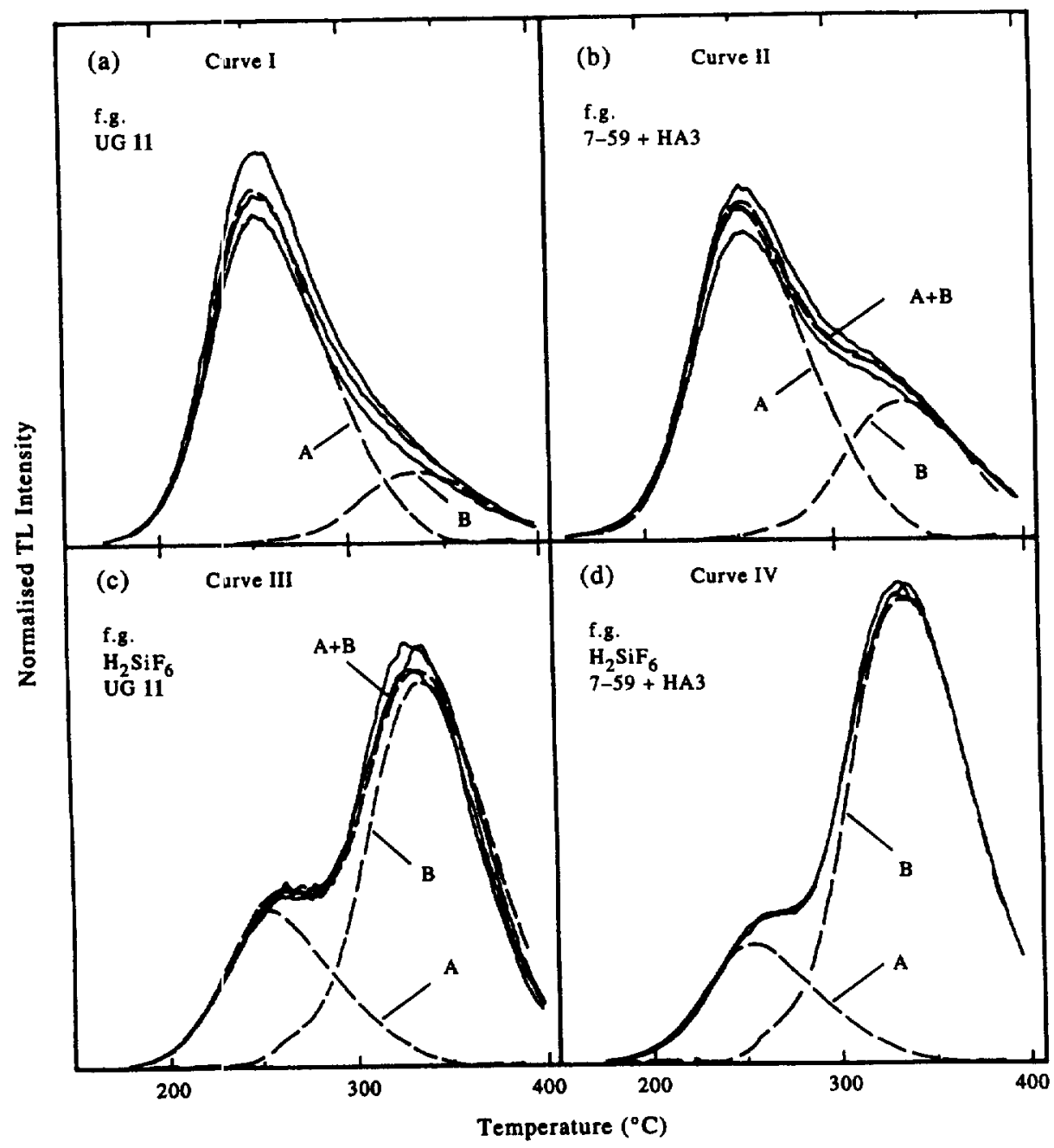

Fig. 17. TL glow curves obtained for a fine-grained sediment using two different filter combinations (Schott UG-11 or Corning $7-59$ plus heat-absorbing filter) (compare left with right) and using two different preparation methods (with and without use of fluorosilicic acid) (compare lower and upper graphs, respectively). Curves can be deconvolved into two luminescence components, A and B (from Debenham, 1987). 
(a)

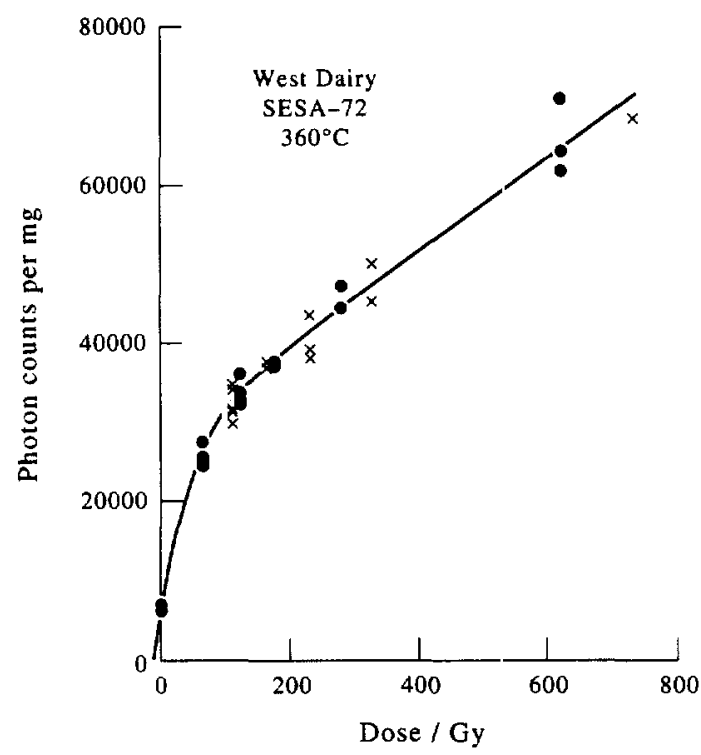

(b)

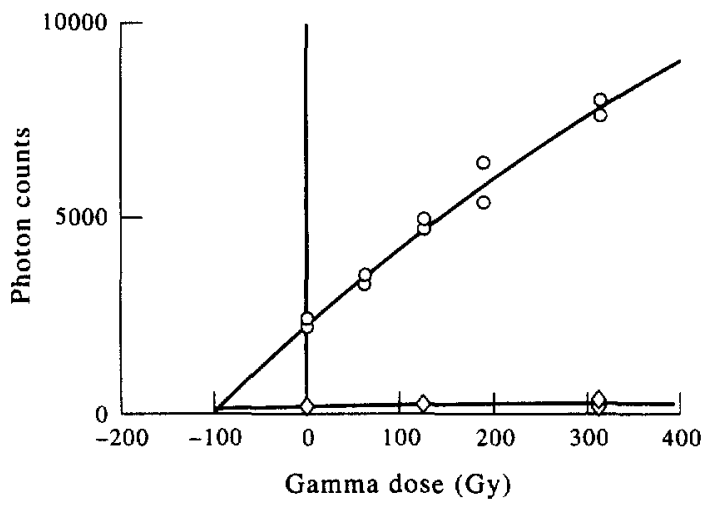

Fig. 18. (a) Combined additive dose $(x)$ and regenerated (O) growth curves for TL at $360^{\circ} \mathrm{C}$ for a quartz from a dune (West Dairy) older than that in Fig. 14 (from Huntley et al., 1994). (b) Additive IRSL data for quart? from the same dune as in (a) (from Huntley et al., 1993a).

what is known as the West Dairy Range in SE Australia. The micro-inclusions are so small $(<10 \mu \mathrm{m})$ that their internal dose rate is negligible and hence the equivalent doses obtiined should be the same as for quartz, provided that both signals are thermally stable. For the 200 ka beach sand this assumption appears to be valid and the equivalent doses are similar. However, Fig. 18(b) shows the far greater linearity for the micro-inclusions, although saturating behaviour can be seen when higher irradiations were given whilst attempting to date the older dune sands which extend back: in age towards 1 million years.

\subsection{Alpha versus beta and gamma}

One phenomenon which appears to have received less attention in recent years is the different TL response to alpha radiation from that to beta and gamma radiation. In simplified terr.ns, the TL from beta and gamma radiation is produced by ionisation caused by the secondary elestrons produced when the high-energy beta particle or gamma ray photon interacts with the crystal. This results in a uniform production of free charge through the crystal. This is compared with the localised delivery of energy associated with an alpha particle moving through the crystal lattice. Ionisation is localised close to the linear track of the al]pha particle and thus the TL along the track is in saturation (Aitken, 1985). This has two effecl.s, namely lower TL produced per unit dose absorbed (leading to the need to measure the alpha efficiency/ relative to beta or gamma efficiency) and a growth curve which remains linear to a higher dose level than for either beta or gamma irradiation, which behave identically (Aitken, 1984).

\subsection{Fine grain versus coarse grain}

Although feldspar separates, particularly potassium-rich feldspar separates, have a higher saturation level than quartz, as discussed in Section 4.4, there appears to be a lower saturation level for finegrain feldspars such as are found in loess (Wintle, 1990). This type of matrix has a dose rate of $\sim 3-5 \mathrm{~Gy} / \mathrm{ka}$, and the TL, OSL and IRSL growth curves show clear exponential behaviour for samples as young as $50 \mathrm{ka}$ (Zhou and Wintle, 1994; Questiaux, 1991). It has not been established whether this relatively early saturation of both the TL and IRSL signals is because of the domination of the luminescence output by a particular feldspar group, e.g. the plagioclase feldspars, and it would be of interest to obtain growth curves for different emission bands.

Differences in thermal stability have been recorded in isothermal experiments on fine-grain loess and on finely crushed potassium feldspar ( $\mathrm{Li}$ and Wintle, 1992). It would also be interesting to compare their growth curves.

In nature, differences might also result from surface weathering effects which would be greater (relative to volume) for the smaller grain size and, in the case of the natural irradiation response, the growth to saturation would be affected by the alpha response as discussed in the previous section. It is interesting to note that neither of these situations is 
relevant to micro-inclusions of feldspar inside quartz grains.

\subsection{Equilibrium below saturation}

Another important consideration in dating older samples relates to the long-term thermal stability of the luminescence signal. An equilibrium between electron trapping from the environmental radiation field, and thermal detrapping depending on the trap characteristics, may be established before the trap reaches saturation. The TL signal from such a sample can always be increased by laboratory irradiation, even though the natural TL signal has become constant during burial. In this situation, no growth curve construction approach can result in the appropriate equivalent dose being derived.

\subsection{Concept of $D_{E}$ plateau}

If a single TL (or OSL) trap could be identified and sufficient thermal stability established, either by laboratory experiments or by the dating of a sample of known age, then the irtegrated TL peak or OSL decay curve could be used for dating. However, many TL peaks are observed in natural materials, and because of the high measurement temperatures (i.e. above room temperature) and the relatively high heating rates (e.g. around $5^{\circ} \mathrm{C} / \mathrm{s}$, so that a large number of measurements can be made in a short time) these TL peaks overlap. Hence, a laboratory procedure was devised to establish the part of the glow curve which had sufficient long-term stability (Aitken, 1985).

4.8.1. $D_{E}$ plateau versus $T$ for assessing thermal stability. The simplest approach developed for heated material was to plot the ratio of the natural $\mathrm{TL}$ and the regenerated $\mathrm{TL}$ response (obtained from a subsequent laboratory irradiation) for a single disc sample. Provided that there has been no non-uniform sensitivity change due to the heating, a plateau will occur above a particular temperature, and the TL output above that temperature can be used for dating.

The next approach was to use two discs and compare the natural TL of one disc with the enhanced signal from the other disc which has received an added dose at least as large as the equivalent dose. This removes the requirement for sensitivity change, but now requires the growth to be linear for all glow curve temperatures. This would be inappropriate for pottery whose TL signals showed supra-linearity around $325^{\circ} \mathrm{C}$ (Flening, 1970).

For samples showing such non-linearity it is necessary to calculate $D_{\mathrm{E}}$ as a function of temperature and use this to juige the region of thermal stability. This, and the simpler ratio plots, were also useful in determining whether the light signals observed on heating contained a component which was not due to the release of trapped electrons, but due to chemical processes on the surface of grains, so-called spurious luminescence (Aitken, 1985).

4.8.2. Anomalous fading and the $D_{E}$ plateau. Another phenomenon which may, or may not, be detected by plateau tests of the type described above is anomalous fading. This is the term used for the reduction in TL signal that is seen in the high-temperature part of the glow curve and which cannot be explained in terms of normal thermal fading. Its presence is detected when irradiated samples are stored for some time before measurement, compared with the signal that would have been obtained immediately after irradiation. It was first observed for feldspars of volcanic origin (Wintle, 1973) but has since been observed for a range of feldspars extracted from pottery and sediments. The behaviour was termed anomalous as it occurred in parts of the glow curve which would be expected to be thermally stable over a period of weeks (namely above $200^{\circ} \mathrm{C}$ ). There is often a weak dependence on glow curve temperature, with fading being greater between $200^{\circ} \mathrm{C}$ and $300^{\circ} \mathrm{C}$, compared with $300^{\circ} \mathrm{C}$ to $400^{\circ} \mathrm{C}$, and this would show up as a poor $D_{\mathrm{E}}$ plateau when TL measurements were made immediately after irradiation.

4.8.3. DE plateau for assessing bleaching of $T L$. When TL measurements were first made on finegrained sediments, bleaching experiments indicated that the TL at higher temperatures was bleached more slowly (Wintle and Huntley, 1980). Modern samples gave finite signals, whose absolute values were greater at higher temperatures. Since this unbleachable residual TL signal would be incorporated in the TL signal of any older sample, it would cause the three earlier tests (Section 4.8.1) to be inapplicable. Instead measurement procedures which involved the estimation of, and allowance for, the residual level were developed (Section 5.4.1); the values of $D_{E}$ were then plotted as a function of temperature, and a poor plateau was taken to be a sign of incomplete bleaching at deposition, which was inadequately allowed for by the laboratory bleaching experiments.

For TL dating of potassium feldspars separated from sediments which are likely to have experienced limited light exposure, Mejdahl (1988b) developed a procedure which involved using several aliquots of sample which had been exposed to light for different lengths of time. The value of $D_{E}$ used for dating was that obtained using the bleached aliquot which gave the best plateau for $D_{\mathrm{E}}$ versus $T$. In this case the residual level for that light exposure was presumed to be equal to that which would have been obtained for the samples at deposition.

4.8.4. $D_{E}$ plateau versus $t$ for assessing bleaching of OSL. OSL and IRSL signals are measured as a decaying luminescence curve as a function of stimulation time, $t$. If the OSL/IRSL signal is made up of more than one component, each with a different 
(a)

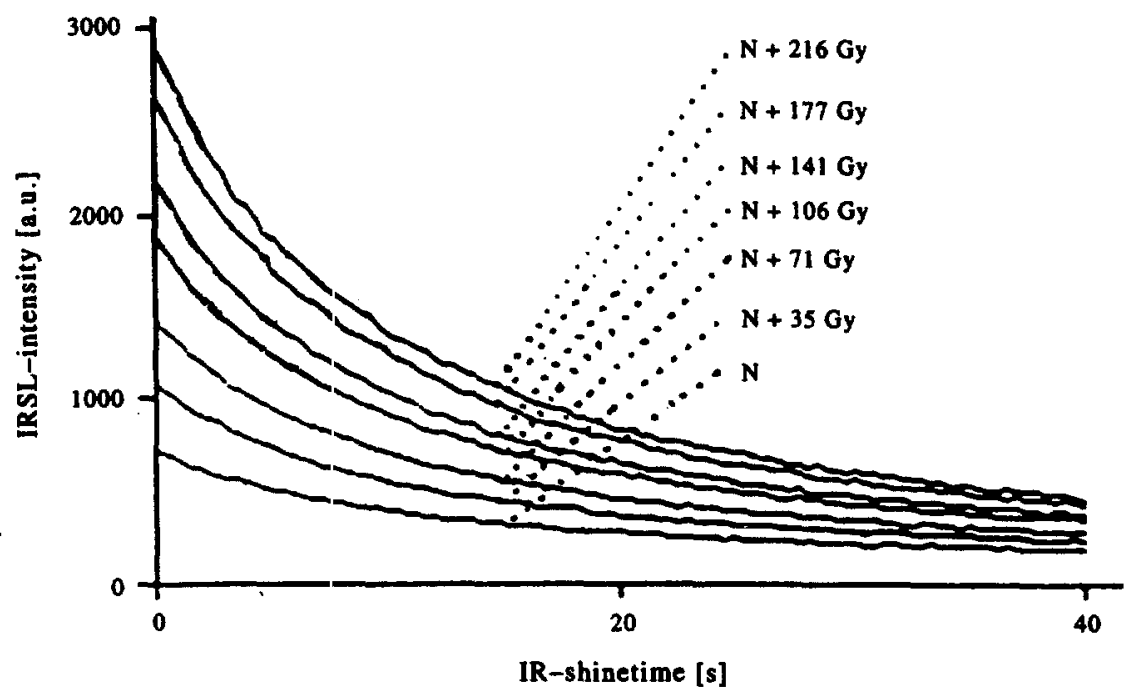

(b)

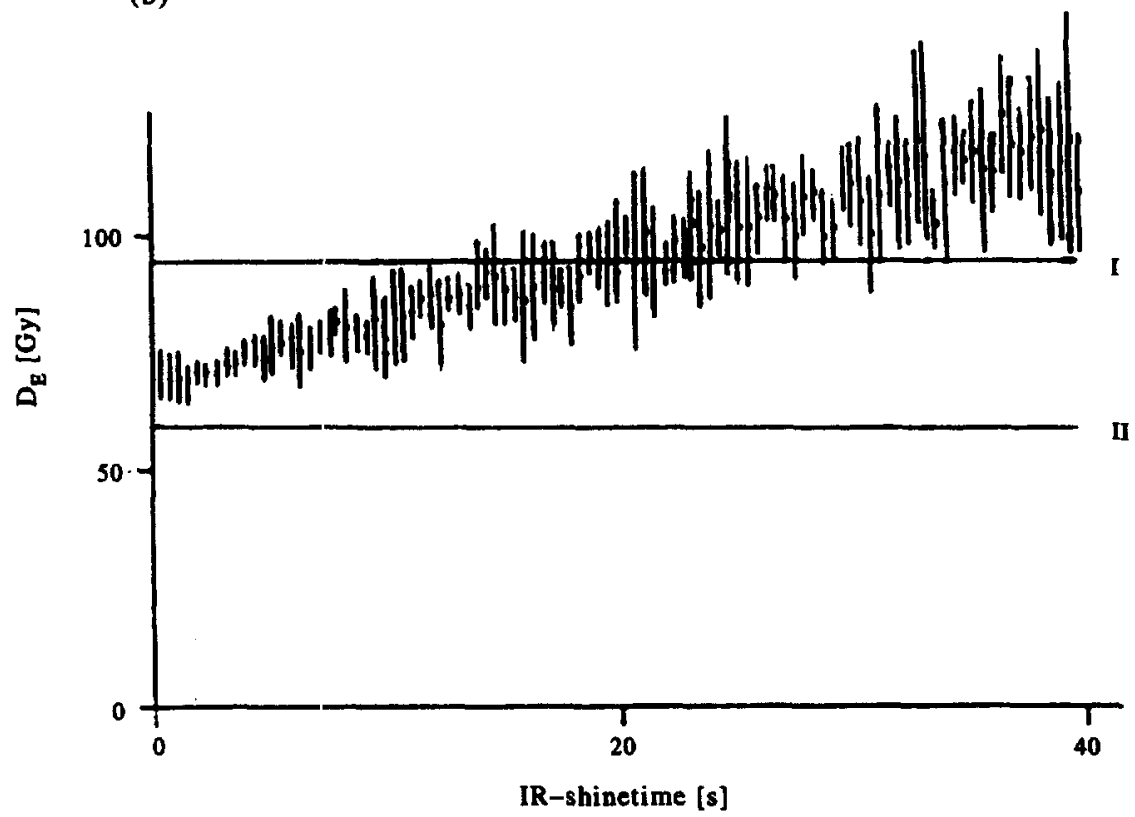

(c)

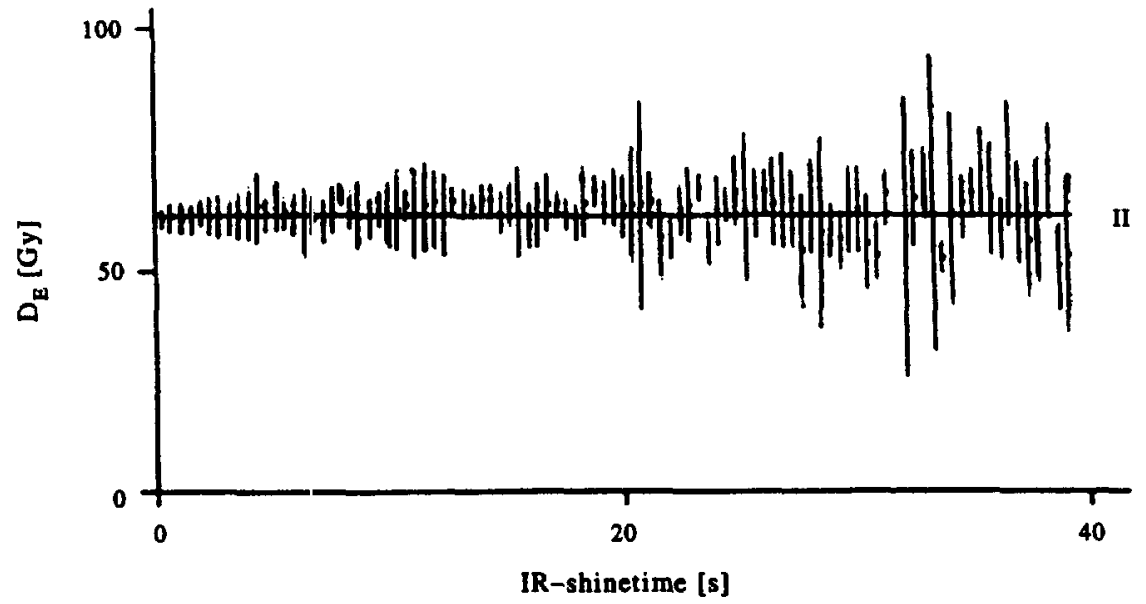

Fig. 19. (a) IRSL decay curve; for fine-grained loess given additive doses of 0-216 Gy obtained for IR exposure times of $40 \mathrm{~s}$. (b) Values of $D_{E}$ obtained for very short time intervals from the data in (a), and showing no plateau of $D_{\mathrm{E}}$. (c) Values of $D_{\mathrm{E}}$ obtained when the mean IRSL intensity for the $50-60 \mathrm{~s}$ interval was subtracted using the technique of Aitken and Xie (1992) (from Lang and Wagner, 1997). 
light sensitivity, then the analysis of the additive dose growth curves obtained for each stimulation time should provide inforrnation on the completeness of bleaching at deposition, as was first suggested by Huntley et a!. (1985). For this to be applicable, the shape of the OSL/IRSL decay curves needs to be independent of dose. For well-bleached samples, which would have zero luminescence at deposition, the value of $D_{\mathrm{E}}$ should be independent of the stimulation time at which it is derived (Aitken, 1992).

Figure 19(a) shows IRSL decay curves obtained for fine-grained loess (Lang and Wagner, 1997) and Fig. 19(b) shows the plot of $D_{\mathrm{E}}$ versus stimulation time. No $D_{E}$ plateau was achieved. However, Fig. 19(c) shows that a plateau is obtained if the mean IRSL intensity for the $10 \mathrm{~s}$ interval (50-60 s) was subtracted from the data for $0-40 \mathrm{~s}$. This subtraction technique, which was devised by Aitken and Xie (1992), also takes account of photomultiplier tube noise and scattered light.

\section{USE OF GROWTH CURVES TO OBTAIN $D_{\text {li: }}$}

Estimates of $D_{\mathrm{E}}$ are achieved by comparing the natural luminescence signcl with those induced by laboratory irradiation. A common way of making this comparison is by the use of dose response curves, often known as growth curves.

\subsection{Multiple aliquots}

For TL dating, in which all the trapped electrons are ejected in the measuraments, each estimate of $D_{\mathrm{E}}$ uses many aliquots of the sample, each consisting of 5-8 $\mathrm{mg}$ of sand-sized grains, of either feldspar or quartz, or $\sim 1-1.5 \mathrm{mg}$ of fine $(4-11 \mu \mathrm{m})$ grains. In each case the grains are spread, or deposited, on $1 \mathrm{~cm}$ diameter aluminium discs for ease of handling and, in the case of TL, for heat transfer. The same approaches are applied to OSL measurements.

5.1.1. Additive dose. In the additive dose procedure, each disc (or group of 4-6 discs) is given a different laboratory dose which results in the enhancement of the luminescence signal. This approach was developed for pottery dating and relies on there being zero signal at $t=0$, i.e. at the time of firing. The assumption is that the sensitivity to laboratory irradiation is the same as it had been for radiation during burial. Figure 20(a) shows that $D_{\mathrm{E}}$ is obtained by extrapolating the signal value to zero, assuming that the rnathematical function for the growth behaviour is known (i.e. is linear, exponential or polynomial).

The strength of this procedure is in the fact that the response to laboratory irradiation has not been altered by any laboratory zeroing procedure, such

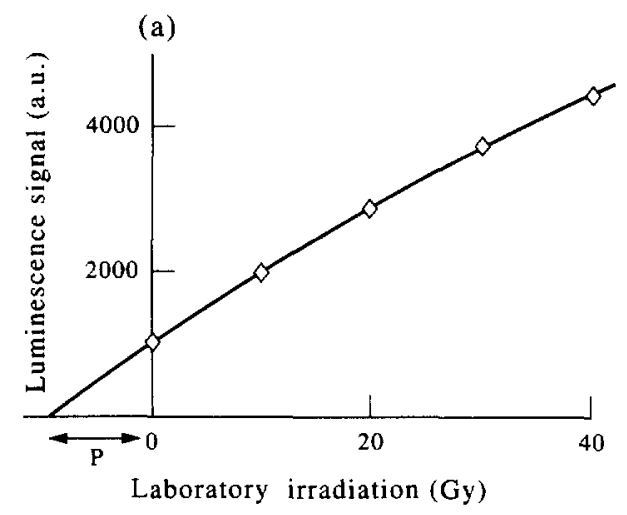

(b)

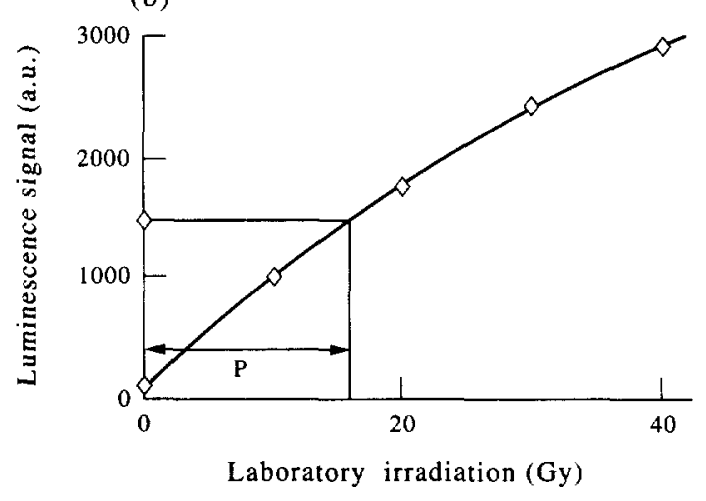

(c)

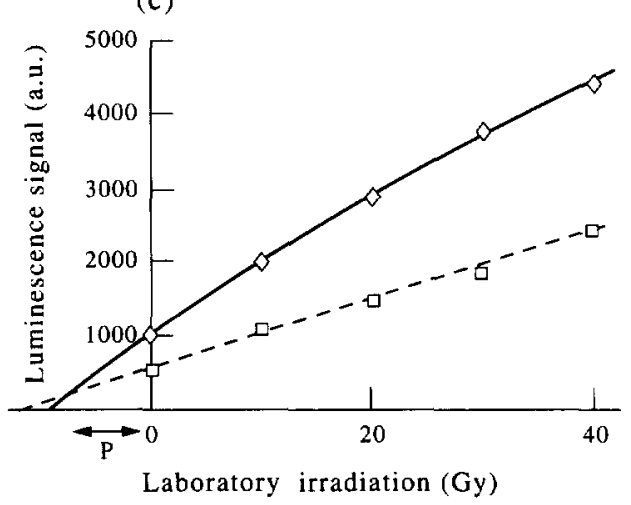

Fig. 20. Schematic representation of the three main methods used for equivalent dose determination (expressed here as the palaeodose, $P$ ); (a) additive dose, (b) regeneration and (c) partial bleach (from Duller, 1996).

as heating, or bleaching with a sunlamp. The main weakness is that it involves extrapolation, with $D_{\mathrm{E}}$ being obtained outside the experimental data set and with the value being dependent upon the mathematical expression used. For samples with poor reproducibility it is difficult to be confident of the most appropriate expression and in any case a large error in $D_{\mathrm{E}}$ will be obtained from the extrapolation.

5.1.2. Total bleach-additive dose. For $\mathrm{TL}$ of sediments it was clear that the extrapolation needed to be made back to a residual level. For samples which were expected to be well bleached, this could be achieved by using a bleached disc for a measured 
residual level, selecting the bleaching time so that longer exposures result in a negligible decrease in the residual TL. For very young samples the residual TL signal will make a significant contribution to the measured natural TL, and this limits the dating of very young samples with this approach. For sediments from less well-bleached erivironments, it is not clear how the appropriate residual level is obtained (see Section 4.8.3).

5.1.3. Regeneration. For pottery, i: is possible to obtain a TL signal by irradiating the grains after they have been heated in order to measure their natural TL. This regeneration TL signal was sometimes found to have a different sensitivity when compared with the additive dose response, in spite of the fact that the grains must have been heated to even higher temperatures, and for longer, during the manufacture of the pottery. Such sensitivity changes were found to be due to both transparency changes (the discs sometimes discoloured on heating) and dose-dependent sensitivity changes connected with particular TL peaks.

In a regeneration protocol, the natural luminescence is compared with very similar light levels resulting from the laboratory irradicition. Thus the precise form of the growth curve, and the mathematical function selected, are unimportant and $D_{\mathrm{E}}$ is obtained by interpolation [see Fig. 20(b)]. This approach will result in a higher precision for the value of $D_{\mathrm{E}}$. The accuracy, however, will depend upon whether there has been any significant sensitivity change as a result of the laboratory zeroing procedure.

5.1.4. Total bleach - regeneration. For sediments which had never been heated, sensitivity changes following TL measurement were even more apparent; instead, the TL signal has been regenerated by irradiating grains which have been optically bleached prior to irradiation. Again, the natural TL was matched to the regenerated signal growth curve in order to determine $D_{\mathrm{E}}$. This method makes two fundamental assumptions, first that the laboratory residual level is appropriate and second that the sensitivity has not been altered by the bleaching, either due to the strength or wave.ength range of the laboratory source. In geographical areas where sunlight is intense and the samples are from aeolian deposits (e.g. Australian and African desert and coastal sands), the sun is the most appropriate light source. For these sands it is also likely that they have been through many bleaching and burial cycles since the grains were released from the bedrock, in contrast to glacial outwash sediments. This multiple cycling is likely to result in a residual level which is similar to that achieved by present-day sunlight bleaching.

5.1.5. The universal growth curve. In geographical areas where the mineral input is likely to remain constant, it might be possible to determine a "universal growth curve" by construeting a growth curve from the addition of doses to discs of a young sample. The natural TL signals of older samples could then be fitted to the curve and their $D_{\mathrm{E}}$ values obtained [Fig. 21(a)]. This approach has been tried for quartz TL from Australian sands (Smith, 1983) and more recently an additive dose master curve [Fig. 21(b)] was obtained for four samples of sand from a rock shelter in Botswana, whose ages ranged from about 20 to about $60 \mathrm{ka}$. The best fit of all the data appeared to be the combined response of a saturating exponential with a linear growth showing up at higher doses. Zhou and Wintle (1994) tried to construct a universal growth curve for loess from China but they concluded that sensitivity changes precluded its adoption.

An approach for quartz grains extracted from loess has been developed by Shlukov and Shakhovets (1987), based on their earlier exper-

(a)

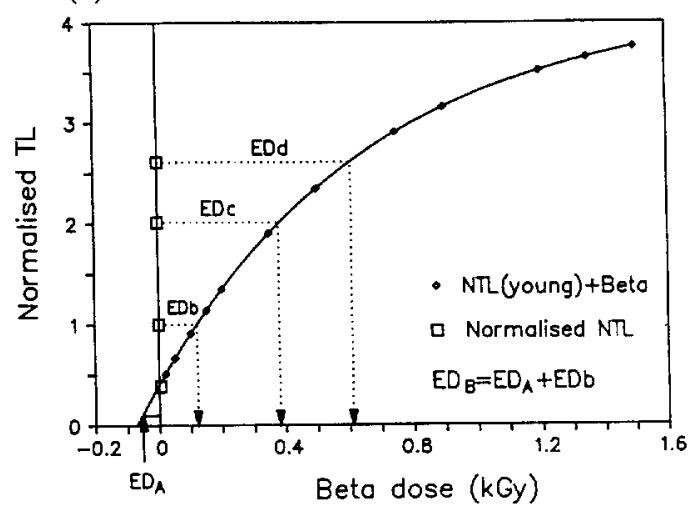

(b)

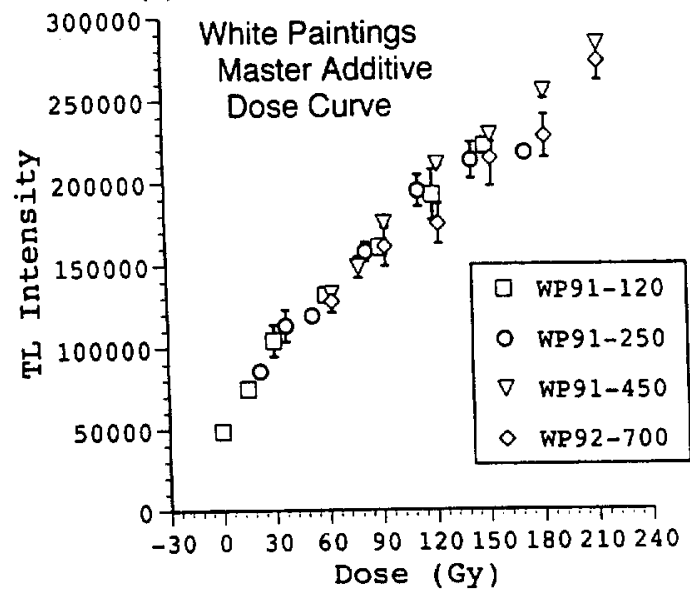

Fig. 21. (a) Schematic diagram of method of using a universal growth curve constructed for a young sample, $A$. The normalized natural TL values for samples $B, C$ and D are matched to the curve to determine their equivalent dose values (from Zhou and Wintle, 1994). (b) Additive dose growth curves for four samples from the White Paintings Rock Shelter, Botswana, which can be overlaid and suggest that a universal growth curve could be used at this site (from Feathers, 1997). 
imental work (Shlukov $t$ t al., 1993). All loess is assumed to have the same saturation value $S_{\infty}$. The radiation sensitivity $\chi$ (in terms of luminescence signal per unit absorbed dcse) is assumed to be the same for all samples and is obtained for a calibration sample whose age has been obtained by the radiocarbon dating method applied to associated material. For individual samples it is only necessary to measure the natural luminescence signal $(S)$, the level reached after $3 \mathrm{~h}$ kileaching with a mercury lamp $\left(S_{0}\right)$ and the in situ dose rate $(E)$ measured in the same way as for the calibration sample. A simple growth towards saturation is assumed and thus the age for the sample is given as

$$
t=(\chi E)^{-1} \ln \left\{\frac{\left(S_{\infty}-S_{0}\right)}{\left(S_{\infty}-S\right)}\right\} .
$$

Results have been reported for parts of Russia and the Ukraine (Shlukov et al., 1997), but it appears that a curve witt. new parameters needs to be established for each area with different source material.

\subsection{Scatter of luminescence signals}

5.2.1. Sources of scatter. There are several reasons why any one sub-sample of grains is not the same as any other, thus causing variations in the amount of luminescence observed from each sub-sample. Firstly, larger amounts of material will result in different amounts of light. It is thus good practice to make measurements $\mathrm{c} n$ equal weights of grains as much as possible, witl weights of $1-5 \mathrm{mg}$ being typical. The number of $100 \mu \mathrm{m}$ grains in a $5 \mathrm{mg}$ sample is about 4000 (ses: Aitken (1985), p. 38) and the number of fine $(4-11 \mu \mathrm{m})$ grains in a $1 \mathrm{mg}$ finegrain sample is much la:ger. Provided that a high precision balance is available, it is possible in theory to use "weight" normalisation.

However, many studies on quartz have shown that relatively few grains are responsible for most of the signal. Using an imaging photon detector, McFee and Tite (1994) found that in four sediment samples the individual TL response of the quartz grains had quite differe.tt behaviour. They found that about $5 \%$ of the grains were significantly brighter than the rest, and of these some had high natural TL and a high second glow TL, whereas others had high natural TL but a low second glow. Such behaviour is thought to be very common. Murray and Roberts (in press a) and Murray et al. (1995) found a wide range of sensitivities for the OSL of single quartz grains.

McFee and Tite (1994) discuss two main reasons why some grains have a high TL or OSL response and a lower response to laboratory irradiation, both relating to the level of dose experienced by the individual grain. This may relate to a problem of bleaching, with a significant residual signal being left in some grains and not others, as would be expected for any grain not transported by the wind.

Huntley and Berger (1995) presented IRSL data obtained for potassium feldspars from two incompletely bleached sediments. They discussed the scatter in terms of two models relating to incomplete bleaching at deposition. In one the light-sensitive traps giving rise to the OSL are not completely emptied in all grains; in the other, traps from which electrons from less light-sensitive traps can be released by the preheat procedure and then trapped in the OSL traps. How these affect normalisation procedures is discussed in Section 5.2.4.

It is important to realise the procedural implications of incomplete bleaching. If it is homogeneous (i.e. all grains are partially bleached to the same degree), then it does not contribute to scatter and the multiple aliquot protocols described earlier are entirely appropriate, but too old an age may be obtained. If, however, bleaching is heterogeneous (i.e. the grains are bleached to differing extents), then each grain will contain a different $D_{\mathrm{E}}$ and only the lowest values of $D_{\mathrm{E}}$ will relate to the sedimentary event of interest. This differential bleaching will directly contribute to scatter, which may then be reduced by using a large number of grains. Unfortunately the average $D_{\mathrm{E}}$ then measured will not be relevant to the depositional event.

A completely different source of scatter is related to the microdosimetry. Such a problem could occur if a grain contains an inclusion which is highly radioactive, such as a small zircon, or if the grains are close to such grains. The dose rate could be lower for some grains rather than others in the case of some grains being coated with calcite, and others not; the two populations of grains would be mixed during sample preparation (Olley et al., 1997).

5.2.2. Normalisation procedures to overcome scatter. In the application of multiple aliquot dating procedures, it is assumed that the grains on any one disc are similar, in terms of the total dose received, to those on any other. If this is the case, then it should be possible to make allowance for slightly different amounts of grains on each disc by weighing each disc and calculating a normalisation factor to be applied to the luminescence signal obtained from each disc. For fine grains, weighing of individual discs is not necessary, since the production of the discs should result in equal weights being deposited. For sand-sized grains, weight normalisation is one of the procedures which has been tried. However, the luminescence output from quartz extracted from sediments has been shown to be highly variable (Huntley et al., 1993a) and the signal from any one disc is dominated by relatively few grains. This has led to high disc-to-disc scatter (even after weight normalisation), as observed in early OSL dating studies on quartz (Rhodes, 1988; GodfreySmith et al., 1988). 
5.2.3. Procedures developed for $T L$. For TL dating, normalisation procedures were developed which utilised the TL signal generated by an irradiation subsequent to the TL measurements made to obtain the additive dose or regeneration growth curve. This is known as "dose normalisation". However, for this procedure to work it was necessary for the "second glow" response to be independent of the dose received by the sample prior to the first TL measurement. If there was a dose-dependent sensitivity change, then the shape of the first growth curve would be altered and an incorrect value of $D_{E}$ obtained. To overcome this, the "equal pre-dose" normalisation procedure was developed (see Aitken (1985), p. 128), in which all discs are arranged to have received the same total dose and two preheats prior to the application of the normalisation dose.

Another normalisation technique developed for TL of pottery is known as "zero-glow monitoring" (Aitken and Bussell, 1979; see Aitken (1985), p. 127) which has been applied to both fine-grain and coarse-grain samples. Before any treatment (preheating, bleaching or large irradiation), the discs are given a small test dose and heated to about $160^{\circ} \mathrm{C}$ to obtain the integrated TL : esponse in the region $100-150^{\circ} \mathrm{C}$, which is then used for normalisation.

5.2.4. Procedures developed for OSL. For OSL dating it is possible to use another type of normalisation procedure, "natural" normalisation, which involves the measurement of the initial part of the natural signal prior to any laboratory treatment. This is also known as "short shine" normalisation, as it involves a short exposure (typically $0.1 \mathrm{~s}$ ) to the stimulation light source, removing $<1 \%$ of the natural OSL (Smith et al., 1990b). Rhodes (1988) reports a reduction of the scatter of data points for a well-bleached sand dune from $\pm 5-15 \%$ to $\pm 2-8 \%$ when using "natural" normalisation instead of weight normalisation.

Since natural normalisation is given before any treatment, a problem could arise if there were grains present which had signals with different thermal stabilities. Stoneham and Stokes (1991) reported a comparison of the "natural" normalisation and "dose" normalisation applied to sediments. For the latter, the normalisation factor obtained was derived from the optically bleached discs which had been given the same beta irraciation and then had their OSL measured for a few seconds; however, different values of $D_{\mathrm{E}}$ were otitained since the normalisation factor was proportional to the dose given in order to construct the addivive dose growth curve.

"Natural" normalisation can cnly be applied when the natural OSL signal is sufficiently large. For samples with low sensitivity, a procedure similar to the "equal pre-dose" normalisation procedure for TL has been applied (see Pye et al., 1995). The
OSL response to a test dose is measured following a series of bleaching and preheat procedures, and this approach is known as "equal total dose" normalisation. A comparison of the results using the method and "natural" normalisation has been reported for a number of young $(<500 \mathrm{y})$ sands from a dunefield in south-eastern California (Stokes et al., 1997). The values of $D_{\mathrm{E}}$ obtained for each method agreed within one standard deviation. For older samples $(\sim 120 \mathrm{ka})$ Wood (1994) reported that "natural" normalisation resulted in less scatter than "dose" normalisation. Even so, his growth curves show considerable scatter (around 20\%). This could result from either unequal and inadequate bleaching of grains, since they are from tsunami deposits and related marine units, or unequal post-depositional irradiation since some of the tsunami sand samples were collected from between boulders in the tsunami-formed deposit. A similar increase in scatter when using "dose" normalisation was reported for fluvial sediments (Perkins and Rhodes, 1994).

For grains in which the main OSL traps are incompletely bleached, "natural normalisation" will result in a normalised additive dose growth curve for which the natural values are very similar, but there is increasing scatter for the higher additive dose points (Duller, 1994b; Huntley and Berger, 1995). Huntley and Berger (1995) also consider another model in which the OSL traps were completely emptied at deposition, but in which there are also traps which are less light sensitive, and which are bleached to different extents. Charge could be transferred from these traps to the OSL traps by a preheat, as reported by Ollerhead et al. (1994). This would result in the same range of scatter for each point (including the natural) on the additive dose growth curve, but Huntley and Berger (1995) had not found such behaviour in their study.

Another normalisation procedure was proposed by Stoneham and Stokes (1991) in which the response of the $110^{\circ} \mathrm{C} \mathrm{TL}$ peak to a test dose was used. This is based on the relationship between the $110^{\circ} \mathrm{C}$ TL peak and the OSL signal. The sensitivity of the $110^{\circ} \mathrm{C} \mathrm{TL}$ peak to a small test dose was measured at the beginning and end of a typical multiple aliquot dating procedure.

In a subsequent study on the same sediment sample, Stokes (1994) used the $110^{\circ} \mathrm{C}$ TL peak response to a small test dose after heating to $200^{\circ} \mathrm{C}$ after every step of a conventional multiple aliquot additive dose procedure. A large dose-dependent sensitivity change in the $100^{\circ} \mathrm{C}$ TL peak was observed as a result of the 16-h preheat employed prior to the OSL measurement. A further set of experiments were reported in which short laser exposures were given throughout a series of irradiations and preheats. Comparison of the two sets of sensitivity measurements led Stokes to conclude that "the $110^{\circ} \mathrm{C}$ TL peak increased after dos- 
ing and preheating, whereas the OSL sensitivity changed only when optical bleaching had preceded the dosing and preheating" (Stokes, 1994). Such behaviour permits additive dose multiple aliquot procedures to be normalised using the "natural" normalisation procedure, as demonstrated by Stokes for preheats at joth $160^{\circ} \mathrm{C}$ for $16 \mathrm{~h}$ and $220^{\circ} \mathrm{C}$ for $5 \mathrm{~min}$. This behaviour would also allow the application of a sirgle aliquot additive dose procedure, similar to that developed for feldspars by Duller (1991). It was also pointed out by Stokes (1994) that the $110^{\circ} \mathrm{C} \mathrm{TL}$ peak could be used to monitor sensitivity changes which occur in regeneration procedures when optical bleaching occurs prior to the laboratory irradiation.

\subsection{Potential problems}

From the discussion in Section 5.1, it is clear that each method has inherent problems, particularly as related to $\mathrm{TL}$ of sediments.

5.3.1. $I_{0}$ is not known. For sediments, the TL level of modern samples, $I_{0}$, is: always finite, and can be changed by exposure to laboratory light sources with intensities and wavelengths different from natural sunlight. It also must be remembered that in some cases (e.g. Narrib desert sand) the grains have coatings of iron and manganese oxides which may alter the wavelength distribution of sunlight reaching the interior of the grains. When laboratory bleaching experiments are carried out, it is usually on grains which have had their outer surfaces etched, for example to remove an external alpha dose contribution (see Fig. 3 inset).

Fortunately $I_{0}$ is found to be zero when the OSL (or IRSL) signals of many modern samples are observed. Zero IRSL signal is likely to be reached in a couple of hours of direct southern African mid-day sun (Fig. 11) o: even less time for quartz OSL (Fig. 10). It is this virtual certainty of zeroing in aeolian environments, and a high probability of zeroing in other depositional environments, that makes optical dating so attractive, particularly for young samples.

5.3.2. Sensitivity changes due to laboratory bleaching. It might be thought that sensitivity changes would be less of a probiem for optical dating. The OSL (or IRSL) could be reduced to an effectively zero level by the light exposure which results in the natural OSL (or IRSL) :ignal. A known laboratory dose could then be given, an appropriate preheat applied, and the OSL (or IRSL) signal could then be re-measured. Use of the green light source or infrared diodes within a Riso TL/OSL reader equipped with a beta source and hotplate would thus allow such measurements to be made under computer control.

However, although such limited spectrum bleaching implies the OSL (or IRSL) traps are emptied, the mineral grains will still contain a large number of electrons in less light-sensitive traps. If the sample is relatively old and these traps are mainly filled, then the probability of an electron being trapped in one of the empty lightsensitive traps might be enhanced for the regeneration measurement. For sediment grains which were well-bleached at deposition, it would appear to be more appropriate to use the bleaching protocol which would have been applied for TL dating, e.g. natural sunlight.

One situation where this is not the case has been reported for incompletely bleached colluvial grains ( $\mathrm{Li}, 1994)$. The TL traps of the feldspar grains from colluvium had not been fully bleached at deposition and thus the sensitivity of the IRSL traps was relatively enhanced, compared to what would have been found if the IRSL signal had been measured after a prolonged bleaching. The sensitivity of the IRSL could be altered by changing the bleaching conditions and for these colluvial samples the most appropriate bleaching prior to IRSL regeneration was using IR diodes.

5.3.3. Uncertainties in curve fitting. For older samples the growth curves become non-linear as electron traps start to fill. The use of linear fitting to the "apparently linear" part of an exponential growth curve can produce large systematic errors (Grün, 1996). For a single electron trap, the response can be represented by a single saturating exponential, $I=A\left(1-\mathrm{e}^{-D / D_{\mathrm{s}}}\right)$ where $D_{\mathrm{s}}$ is the parameter which characterises the dose level at which saturation occurs. However for natural samples, a TL signal from a single electron trap is never observed. In spite of this, the most common curve fitting procedure involves the use of a single exponential function, presumably on the assumption that close to the observed saturation value for the mineral mixture, the signal will be dominated by a particular trap (e.g. Lamothe et al., 1994).

Some data sets appear to be better fitted by the combination of an exponential and a linear growth term $I=A\left(1-\mathrm{e}^{-D / D_{s}}\right)+B D$, where the linear component continues to grow after the exponential component has saturated (Grün and McDonald, 1989). For young samples which exhibit supra-linear growth at low doses, other functions have been applied (Grün and McDonald, 1989). A more general empirical approach involves the use of polynomial functions (Guibert et al. (1996), Berger (1996), and references therein).

The choice of a curve fitting procedure and appropriate weighting of data points for a particular data set is probably the major cause of differences in $D_{\mathrm{E}}$ that would be obtained by different laboratories, when using an additive dose procedure. These problems do not apply when regeneration procedures are used, since $D_{\mathrm{E}}$ is obtained by interpolation of the data set, making the choice of a particular growth function unimportant. It is thus 
important to bear in mind the possible choice of curve fitting procedures when ccmparing dates obtained by different research groups.

\subsection{Multiple aliquots - other approaches}

More sophisticated methods for determination of $D_{\mathrm{E}}$ have been developed to overcomie the problems related to optical bleaching (Section 5.2) and problems of non-linearity (Section 4.2).

5.4.1. The partial bleach method. This approach was developed for TL dating of sediments for which it appeared that the TL signal at any particular temperature could be divided nto a "bleachable" and an "unbleachable" component (Wintle and Huntley, 1980). For dating it w'ould be necessary to separate the bleachable component that had built up since deposition. The philosophy behind this approach is clearly laid out by Huntley (1985). It is also a necessary requirement that the bleachable signal is reduced by the light source in a way which does not depend upon the dose that the grains have previously received. The applicability of this method is also critically dependent on the nature of the bleaching process, i.e. whether grains are homogeneously or heterogeneously bleached (see Section 5.2.1).

An idealised data set that would be obtained by the partial bleach is shown in Fig. 20(c). One half of the prepared discs are irradiated and measured as in the production of the additive dose growth curve. The other half received the same irradiations, but are then exposed to a light source of relatively low intensity which had its spectrum restricted to the less energetic wavelengths. The aim of this bleaching is to reduce the bleachable component of the TL from each disc by a fixed percentage. The two lines will then intersect at a point whose coordinate on the dose axis is $D_{\mathrm{E}}$. This approach has proved powerful in a variety of depositional environments and useful back to several hundred thousand years (Berger et al., 1992).

A similar philosophy was applied to IRSL from a fine-grained alluvial deposit (Fuller et al., 1994). Using IRSL it is possible to use a single set of irradiated discs for all the measurements, provided that the IRSL is measured for a short time. Using a 0.5$s$ infrared exposure, a set of partial bleach curves was determined with the signal being reduced by increasing exposure to a light source with output restricted to wavelengths from $50 C$ to $660 \mathrm{~nm}$ (e.g. using a SOL2 light source with a Schott BG-39 and a Corning 3-67 filter combinationi. This approach is based on the assumption that the IRSL signal is made up of more than one component, each with a different sensitivity to light. In the example of fluvial sands from Spain which were dated using this procedure (Fuller et al., 1996), it appears that the samples were actually well blec.ched, since the analysis resulted in low residual levels for each sample.

5.4.2. The selective bleach method. The bleachability of the $325^{\circ} \mathrm{C}$ TL peak in quartz and the characterisation of its emission spectrum have been put together to form the basis of the selective bleach method (Prescott and Mojarrabi, 1993). The TL of one set of natural and irradiated samples is measured using a Schott UG-11 and Corning 7-59 filter combination (Franklin and Hornyak, 1990), so that the $380 \mathrm{~nm}$ emission (Section 2.1.2) is enhanced relative to the wavelength emission of the $375^{\circ} \mathrm{C}$ TL peak (Section 2.1.1). The TL of another identical set of discs is measured after bleaching with wavelengths longer than $475 \mathrm{~nm}$ (e.g. by placing a Chris James 101 yellow gel filter in front of a sunlamp) (Section 3.2). The difference between the two sets of glow curves will permit the construction of the growth curve of the $325^{\circ} \mathrm{C}$ TL peak. Given the close relationship between the $325^{\circ} \mathrm{C}$ TL and the OSL signal from quartz, it would now appear preferable to use the OSL measurements of this trapped electron population. However, good examples of the application of the selective bleach method are presented by Smith et al. (1997).

5.4.3. The "Australian slide" method. Given the behaviour of the $375^{\circ} \mathrm{C}$ TL peak observed for quartz from Australian dune sands [Fig. 18(a)], it was necessary to find a curve fitting procedure which used the regeneration data set to determine the shape of the growth curve. In the "Australian slide" method, this regeneration curve is moved along the dose axis until it overlaps the additive dose data set. The dose shift required to match the two data sets will give $D_{\mathrm{E}}$. The software that has been developed to perform this shift makes allowance for a sensitivity change between the two data sets, although this is not necessarily advocated (Prescott and Robertson, 1997). For quartz grains which have been through many cycles of irradiation and bleaching, this sensitivity change appears to be small. The philosophy behind this protocol is expanded in Prescott and Robertson (1997). For the SE Australian dune sands the high-temperature quartz growth could be fitted by the combination of a straight line and a saturating exponential (Huntley et al., 1994).

\subsection{SARA}

Regeneration procedures have long been recognised as being able to provide precise estimates of the dose required to give a laboratory-produced luminescence signal which matches the natural luminescence signal. However, it was known that in many cases, sensitivity changes prevented that value of the dose from being equal to the equivalent dose, $D_{\mathrm{E}}$. This is true of both sediments and heated materials. A method which exploits the precision avail- 
able from regeneration measurements, but allows for the sensitivity change, was developed for young archaeological materials kiy Mejdahl and BøtterJensen (1994) and was called SARA, the Single Aliquot Regeneration on Additive Dose method. Although the words "single aliquot regeneration" appear in the name of this protocol, the method is not a true single aliquot method, as at least four discs are required.

In this procedure, which uses a relatively small number of discs, the natural luminescence is compared with a short growtla curve constructed with three regeneration doses, one to give a luminescence signal slightly above the ratural luminescence, one slightly below it and one very close to it. The interpolated dose $D_{0}$ that matches the natural dose is obtained. In the case of pottery, the discs were heated to erase the natural TL; in the case of GLSL and IRSL measurements, light exposures of $100 \mathrm{~s}$ were applied to erase the natural signal. The same regeneration procedure is used for other discs which have received additional doses $(B 1, B 2, B 3)$ on top of their natural dose. For these discs values of $D 1$, $D 2$ and $D 3$ are obtained. By plotting $D_{0}, D 1, D 2$ and $D 3$ as a function of added dose, a straight line graph is obtained which can be extrapolated to the dose axis to obtain $D_{\mathrm{E}}$. The dose values $D_{0}, D 1, D 2$ and $D 3$ may be incorrect owing to sensitivity changes. Provided that the sensitivity change is independent of the applied dose, $D_{\mathrm{E}}$ obtained in this way will be the true equivalent dose.

Further tests of the SARA method have been undertaken, both using the TL signal of archaeological samples (both quartz and feldspar) and the green light stimulated luminescence (GLSL) of young geological sediments (both quartz and feldspar) (Mejdahl and Bixter-Jensen, 1997). The SARA results were compared with TL or IRSL ages obtained by conventional multiple aliquot procedures and the agreement was excellent. The reproducibility was found to be improved if an additional preheat was applied after each OSL measurement and before the subsequent regeneration dose was give. Mejdahl and Brtter-Jensen (1997) used a $40 \mathrm{~s}$ preheat at $220^{\circ} \mathrm{C}$ for both quartz and feldspars. An advantage of the SARA method is that it also makes allowance for supra-linearity, which is difficult to correct for using the multiple aliquot procedure when there is a substantial sensitivity change.

\subsection{Modified SARA}

Murray (1996a) extended the SARA method as described by Mejdahl and Bøtter-Jensen (1994) in his study of the OSL and PTTL of quartz from very young fluvial samples. The original SARA protocol was inapplicable on these samples as they required very long bleaching times between ir- radiations to reduce the OSL to a level which contains no evidence of the previous dose history of the sample (Murray, 1996b). To overcome the effects of this changeable baseline, two slightly different regeneration doses are added to two discs which have identical initial irradiations. The doses are selected so that one results in an added dose OSL integral which is slightly higher than the natural OSL integral and one which is slightly lower, thus allowing an interpolated value of the matching dose to be determined. These interpolated dose values are then plotted as in the normal SARA method. This approach was used by Murray et al. (1995) and by Olley et al. (in press) for studies of fluvial sediments.

\section{PREHEATING}

After irradiation or bleaching, but before the TL, OSL or IRSL is measured, it is usual to hold the sample at a temperature above room temperature for some period of time. There are several reasons for such preheating, and some laboratory protocols involve more than one preheat. Alongside the choice of the optical detection filter, choice of preheat protocol for the particular luminescence signal is probably the most contentious issue in the production of dates. Preheats have been selected with three main objectives in mind, and I will illustrate these with examples from Volume 13 of Quaternary Science Reviews (Quaternary Geochronology), the proceedings of the $7^{\text {th }}$ TL International Specialist Seminar on TL and ESR Dating.

\subsection{To reduce the effect of anomalous fading}

Anomalous fading of feldspars can be observed to occur at room temperature over a period of weeks to months. This has been observed for both TL (Wintle, 1973), OSL (Spooner, 1992) and IRSL (Spooner, 1992) of feldspars. Spooner (1994a) reported anomalous fading of the IRSL of a large number of feldspars when they were measured during storage at either 10 or $100^{\circ} \mathrm{C}$ for 15 months. The luminescence signal decays logarithmically with time, a behaviour which can be expected on theoretical grounds (Visocekas, 1985). The rate of fading has been shown to be more rapid at elevated temperatures, and protocols for TL dating of feldspars often include a relatively long preheat (Clark and Templer, 1988). Mejdahl and Christiansen (1994) advocate keeping both natural and irradiated samples at $100^{\circ} \mathrm{C}$ for 1 week before measurement. Shorter high-temperature preheats of $5 \mathrm{~h}$ at $160^{\circ} \mathrm{C}$ were used by Forman et al. (1994a) to isolate the thermally stable IRSL signal from their fine-grained high Arctic marine sediments; however, this did not prevent anomalous fading of up to $20 \%$ on subsequent storage at room temperature for 30 days. 


\subsection{To isolate traps with adequate thermal stability}

6.2.1. Fine-grain $T L$. For TL dating of finegrained mixed-mineral samples it is particularly important to remove laboratory-induced thermally-unstable TL (Berger, 1995). The TL peaks overlap and in general the lower temperature peaks have a higher sensitivity (Berger et al., 1992), perhaps related to thermal quenching of luminescence centres (Poolton et al., 1995) as the feldspar temperature increases. The effect of preheating at $115^{\circ} \mathrm{C}$ for 4 days for a sample from a lake core in Alaska is shown in Fig. 22; using this preheat a plateau for $D_{\mathrm{E}}$ of $36.7 \mathrm{~Gy}$ was obtained from $230-330^{\circ} \mathrm{C}$, resulting in an age of $11.6 \pm 1.3 \mathrm{ka}$, some $3.4 \mathrm{ka}$ younger than the calendar-correcter radiocarbon date of $15.6 \mathrm{ka}$. However, no single preheating recipe could be applied to all the sarnples from the core. In a wider ranging discussion of fine-grain samples, Berger and Anderson (1994) concluded that no single preheat procedure could ever be successful and that different preheating procedures should be tried until a $D_{\mathrm{E}}$ plateau is obtained; only then is an accurate value of $D_{\mathrm{E}}$ available for an age calculation.

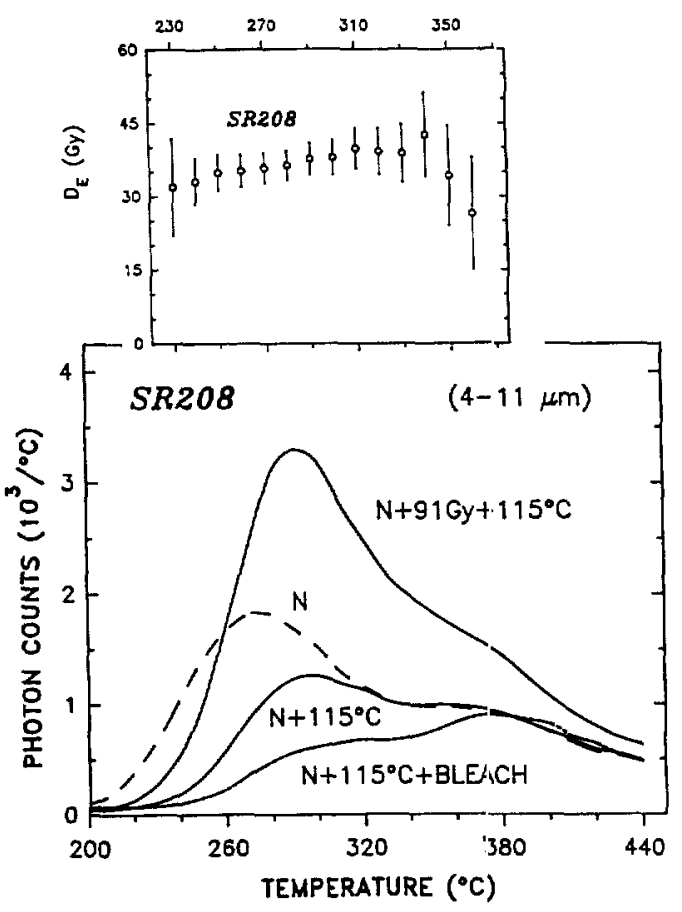

Fig. 22. Glow curves for a fine-grained sample from a lake core in Alaska showing the effect of a 4-day preheat at $115^{\circ} \mathrm{C}$ on the natural TL $(N)$ and permitting comparison with similarly treated irradiated $(N+91 \mathrm{~Gy})$ and bleached sample discs; the latter were given a light exposure in the wavelength range 560 to $800 \mathrm{~nm}$ for 3 days, as part of the partial bleach method of $D_{\mathrm{E}}$ determination. Upper inset shows the values of $D_{\mathrm{E}}$ obtained as a function of glow curve temperature (from Berger and Anderson, 1994).
6.2.2. OSL of quartz. The need for a preheat after irradiation was demonstrated by GodfreySmith (1994) who monitored the decay of OSL signals over a 68-day period, obtained when doses of 100 or $200 \mathrm{~Gy}$ are given in addition to the natural dose to samples of both quartz and potassium feldspars. For quartz the OSL seemed to be made up of a short-lived component which decays in a few days at ambient temperature, and a stable component.

For OSL dating it is more difficult to select a preheat since the thermal stability of the different populations of electron traps giving rise to the room-temperature OSL signal after irradiation is not known. Two preheat procedures have been used extensively for multiple aliquot dating procedures, namely $5 \mathrm{~min}$ at $220^{\circ} \mathrm{C}$ as originally developed by Rhodes (1988) and $16 \mathrm{~h}$ at $160^{\circ} \mathrm{C}$ as developed by Stokes (1992). The former procedure is suitable for an automated system, such as in single aliquot procedures (see Section 7), whereas the latter is not. If the OSL is derived from only the $325^{\circ} \mathrm{C}$ peak, and if the trap depth and frequency factor values of Wintle (1975) are correct, then both preheats would have only a small effect on the OSL signal, a loss of $16.9 \%$ for the $5 \mathrm{~min}$ at $220^{\circ} \mathrm{C}$, and $12.5 \%$ for $16 \mathrm{~h}$ at $160^{\circ} \mathrm{C}$ (Stokes, 1996a). The equivalence of the preheat for the reduction in the natural OSL was demonstrated with data for 28 different sediment samples for which a reduction of $10-20 \%$ was found for most samples.

However, Roberts et al. (1993, 1994b) present evidence for significantly different values of $D_{\mathrm{E}}$ being obtained for 5 samples when using the two preheats. A more recent intercomparison using these same preheats has given values of $D_{\mathrm{E}}$ for 45 samples with ages ranging from a few hundred to about 125000 y (Stokes, 1996b). No significant difference was found between the two preheat procedures for which the weighted mean ratio of $D_{\mathbf{E}}$ values was $0.95 \pm 0.14$.

6.2.3. For feldspar OSL and IRSL. For feldspar OSL Li (1991) suggested two possible preheats, $10 \mathrm{~min}$ at $220^{\circ} \mathrm{C}$ and $5 \mathrm{~h}$ at $160^{\circ} \mathrm{C}$ on the basis of three criteria: (i) a plateau being obtained for the ratio of the natural and irradiated signais, (ii) similarity of the thermal decay rates for the two signals and (iii) the similarity of the shape of the TL glow curves of the natural and irradiated samples after such a preheat.

$\mathbf{L i}$ and Wintle (1992) demonstrated the similarity for loess from many sites around the world of the thermal decay on storage at $220^{\circ} \mathrm{C}$ of IRSL resulting from laboratory irradiation. However, the IRSL signal from $\mathrm{K}$ feldspar separates appeared to have greater stability. They also characterised the IRSL for loess by observing the reduction in signal brought about by heating to successively higher temperatures and concluded that, once the sample had been heated to $350^{\circ} \mathrm{C}$, no IRSL signal was 
observed. Duller (1994a) took this a significant step further, using smaller incremental temperatures to improve resolution, and showed that natural and irradiated samples have different responses, unless a preheat (e.g. $220^{\circ} \mathrm{C}$ for $10 \mathrm{~min}$ ) is applied. The data indicate that the stable IRSL is related to a trap which has a similar thernal stability to the $350^{\circ} \mathrm{C}$ peak, but no one-to-one correspondence has been found between the IRSL signal and a recorded TL peak.

\subsection{To redistribute charge}

In a recently zeroed mineral, radiation results in charge being trapped according to the relative capture cross-sections of the traps. During irradiation in nature, charge will be trapped at and lost from shallow traps, and some of this charge may be retrapped in the trap giving rise to the OSL signal. It may thus be important in the dating procedure to employ a preheat which will cause the equivalent charge transfer after laboratory irradiation (Rhodes, 1988).

\subsection{To equalise sensitivity changes}

Although sensitivity changes are clearly seen when thermal activation characteristic (TAC) curves are measured by pulse heating to temperatures above $250^{\circ} \mathrm{C}$ (Wintle and Murray, 1997), it is also possible that similar activation could have occurred over long time periods at high ambient temperatures, such as in deserts where the surface temperature may reach $70^{\circ} \mathrm{C}$ every day. Indeed, for one Australian sedimentary quartz, TAC curves for natural and laboratory irradiated samples have been shown to be substantially different (Wintle and Murray, in press). For this sample, correcting the change in luminescent recombination probability (i.e. sensitivity) as a function of preheat temperature almost completely removed the previously convincing evidence for thermal transfer in the laboratory irradiated aliquot. This observation has since been repeated on a number of Scandinavian sedimentary and heated quartz samples (Murray, personal communication). Hence giving both natural and irradiated discs the same preheat is a means of equalizing any possible sensitivity changes of this type.

\subsection{A check for adequacy of preheat $-D_{E}$ versus $T$}

6.5.1. For $T L$. Since heating to higher temperatures results in the emptying of progressively more thermally stable traps, a plateau of $D_{\mathrm{E}}$ versus $T$, covering several TL peaks, is the best way of ensuring that the correct value of $D_{\mathrm{E}}$ is obtained. This approach was taken for fine grains (discussed in Section 6.2.1) but is bet1er demonstrated for a sedi- mentary quartz from the archaeological site of Nauwalabila I in northern Australia (Roberts et al., 1994a). A plateau was obtained [Fig. 23(a)] across three peaks $\left(280,325\right.$ and $375^{\circ} \mathrm{C}$ at $\left.5^{\circ} \mathrm{C} / \mathrm{s}\right)$ from 240 to $430^{\circ} \mathrm{C}$, giving a TL age of $28 \pm 2 \mathrm{ka}$. This confirmed both that the stability of all three peaks was adequate for dating a sample of this age and that the grains were well bleached at deposition, since both the 325 and the $280^{\circ} \mathrm{C}$ peaks bleach more rapidly than the $375^{\circ} \mathrm{C}$ peak. This criterion for bleaching of both the 325 and the $375^{\circ} \mathrm{C}$ peaks has been discussed further for a suite of quartz samples from fluvial sands in Australia (Price, 1994).

6.5.2. For $O S L$ of quartz. For the Nauwalabila I sample discussed in the previous section, OSL dating was also carried out on another set of discs which had been preheated for $5 \mathrm{~min}$ at $220^{\circ} \mathrm{C}$. This preheat was shown to erase the optically-sensitive peak at $280^{\circ} \mathrm{C}$ [Fig. 23(b) inset]. $D_{\mathrm{E}}$ was obtained for the integral over the first $500 \mathrm{~mJ}$ of laser exposure [Fig. 23(b)].

For OSL it is impractical to use multiple aliquots to obtain sufficient measurements of $D_{\mathrm{E}}$ as a func-

(a)

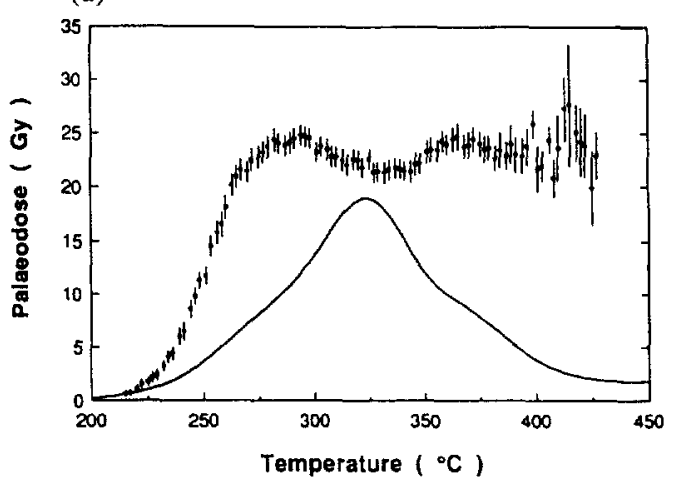

(b)

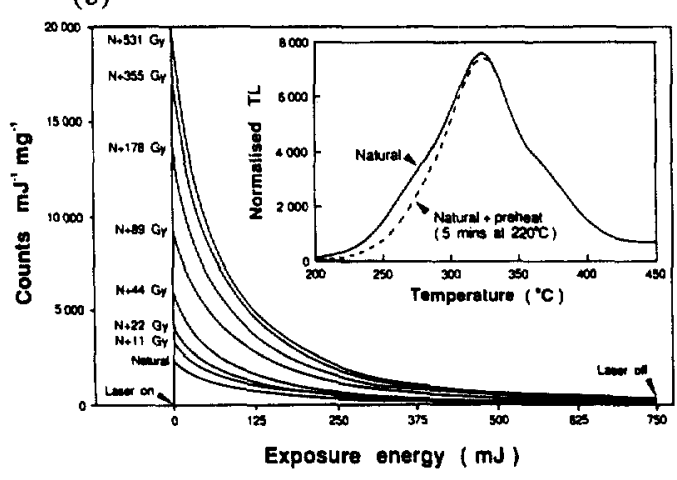

Fig. 23. (a) Natural TL glow curves and plot of $D_{\mathrm{E}}$ against temperature for a sample of Australian sedimentary quartz from Nauwalabila I. The plateau extends upwards from $240^{\circ} \mathrm{C}$, suggesting both thermal stability and efficient bleaching at deposition (from Roberts et al., 1994a). (b) OSL decay curves obtained after a preheat at $220^{\circ} \mathrm{C}$ for $5 \mathrm{~min}$, which removed the $\mathrm{TL}$ at $280^{\circ} \mathrm{C}$ (see inset). Using these decay curves, the value of $D_{\mathrm{E}}$ was found to be $24.6 \pm 0.7$ Gy (from Roberts et al., 1994a). 
tion of preheat temperature to obtain a meaningful plateau. Instead, a procedure equivalent to the simple TL plateau test (Section 4.8 ! was carried out by Rhodes (1988) in irradiated and unirradiated natural aliquots preheated for $5 \mathrm{~min}$ at different temperatures from 160 to $280^{\circ} \mathrm{C}$. The $(N / N+\beta)$ OSL ratios showed stability for $5 \mathrm{~min}$ at temperatures of $220^{\circ} \mathrm{C}$ and above.

\subsection{OSL measurement at elevated timperature}

6.6.1. Feldspars. Another use of heating in OSL measurement procedures relates to the temperature at which the measurement is made. The measurement can be made at room temperature, but this may not be well controlled. Several papers have presented data on the variation of IRSL and OSL signals as a function of the measurement temperature. It is possible to measure the luminescence for a fraction of a second whilst heating up the sample in what would otherwise result ir a normal glow curve. Duller and Wintle (1991) showed that the IRSL signal obtained from a feldspar increased as the temperature was raised from 50 to $200^{\circ} \mathrm{C}$ and then decreased as the trap was emptied with increasing temperature. The increase above room temperature has been used to measure the energy of thermal assistance involved in the production of IRSL, which involves low energy photons in the stimulation (Hütt et al., 1988; Bailiff and Poolton, 1991). It is now relatively common for IRSL measurements to be made at $51^{\circ} \mathrm{C}$ to obtain a reproducible signal which is also slightly higher than that at room temperature. However, it is possible to increase the signal by as much as a factor of 5 by measuring at even higher temperatures, provided that an appropriate filter is used so that small shifts in peak emission wavelength do not have an adverse effect (Duller and Bøtter-Jensen, 1997). This study also demonstrates that when measurements are carried out at elevated :emperatures, the luminescence centres may also be affected by another thermally activated process, namely thermal quenching, as pointed out by Poolton et al. (1995).

6.6.2. Quartz. Smith et al. (1990b) suggested that the OSL signal from sedimentary quartz could be increased by making the measurement at an elevated temperature and reported a dating study using measurements made at $100^{\circ} \mathrm{C}$. In a more recent study Murray and Wintle (1997) conclude that it would be an advantage to measure OSL of quartz at a temperature of $125^{\circ} \mathrm{C}$, so that the trap(s) responsible for the $110^{\circ} \mathrm{C}$ TL peak are kept empty. Measurement at this temperature increases thermal assistance and results in the majority of the OSL being emitted in the first $10 \mathrm{~s}$ of green light exposure in a Risø OSL/TL reader. This also results in a smaller contribution of an OSL signal with a long decay constant under optical stimulation, and would be advantageous for younger samples. A $10 \%$ reduction in integrated OSL due to thermal quenching was observed compared to stimulation at room temperature, but this is outweighed by the other improvements.

\section{SINGLE ALIQUOT PROTOCOLS}

\subsection{Introduction}

In Section 5.1, the procedures used to obtain the equivalent dose involved the use of 24 to 50 sample discs. For TL dating this was necessary because the measurement of the TL signal is destructive, since all electrons are ejected from the traps during heating. The large number of discs used was required to define the growth curve with sufficient precision to enable its extrapolation to be meaningful for the additive dose method. This philosophy was carried over into the new optical dating methods, although even in the first paper on the topic, Huntley et al. (1985) recognised that it should be possible to obtain an estimate of $D_{\mathrm{E}}$ using a single portion of sample. As with multiple aliquot procedures, two approaches could be taken:

one in which the natural OSL could be compared with the OSL resulting from a known laboratory irradiation; this would be a regeneration method, probably using optical bleaching to zero the signal before delivery of a laboratory radiation dose.

the second would use only the initial part of the OSL signal as the measurement, and additional doses would be given to increase the OSL signal. These would be used to construct an additive dose growth curve, as first suggested by Smith et al. (1986).

In both procedures a preheat would be required, as outlined in the previous section, and in the second approach some form of correction would need to be applied to allow for the repeated preheats that a sample disc would experience.

Single aliquot procedures have a number of theoretical and practical advantages.

1. The value of $D_{\mathbf{E}}$ obtained for each aliquot has a very high precision. This is to be expected since the natural response of each aliquot is compared with its individual response to laboratory irradiation. The error term is given by the fitting program used, and even for additive dose procedures the fitting error is typically $\pm 4 \%$ (e.g. Wintle et al., in press). For samples that have been well zeroed by the event that is being dated and that have been in a uniform radiation environment since deposition, the values of $D_{\mathrm{E}}$ are very close. For pottery these requirements are met and typically the standard deviation in $D_{\mathrm{E}}$ between aliquots is less than $5 \%$. For K-rich feldspars from sands ranging from 300 to $600 \mathrm{y}$, 
the standard error in the mean of 18 aliquots was found to be $5-7 \%$ (Wintle et al., in press). The size of the standard deviation can be used to evaluate whether all the grains have been well bleached or not (Li, 1994; Clarke, 1996) and the range in $D_{\mathrm{E}}$ has also been used to demonstrate incomplete bleaching (Murray et al., 1995) and inhomogeneity of the radiation environment for aeolian grains trapped in a carbonate matrix (Olley et al., 1997).

2. Very small amounts of the mineral of the appropriate grain size need to be extracted. This is important for archaeological samples of finite size, such as a piece of pottery or burnt stone. It is of even greater importance for single items such as a wasp's nest forming on the surface of a piece of rock art (Roberts et al., 1997). For very young sediments it is also important when using $\mathrm{K}$ feldspar, which may make up as little as $3 \%$ of the bulk sediment, for dating coastal dunes from Britain and Ireland (Wintle et al., in press). In the ultimate application, single aliquot dating can be applied to single grains, provided that their sensitivities are high enough. Lamothe et al. (1994) have performed such studies on paired feldspar grains and Murray and Roberts (in press b) have made similar studies on single grains of Australian sedimentary quartz.
3. No normalization procedures are required to allow for variations in the number of grains on each sample disc, or different disc-to-disc sensitivities.

4. Procedures for preheating, bleaching and irradiation, as well as the OSL measurement, can all be performed readily within an automated reader. This also increases precision, as there is less opportunity for inadvertent light exposure or loss of grains. For each single aliquot additive dose technique, the laboratory irradiation time is considerably less than that for dating with a multiple aliquot technique, when the same radiation source is used; this results from the cumulative nature of the irradiation schedule in the single aliquot procedure. However, in reality a number of repeat measurements are made and allowance must be made for the preheating times (often $10 \mathrm{~min}$ ). For pottery samples with high reproducibility it would be possible to use a limited number of aliquots. However, for sediments, many more discs (usually 12 to 18 ) are used in order to estimate the scatter. Also, the relatively young ages of pottery (usually $<3000$ y) require shorter irradiation times, compared with most sediments (usually $\gg 1000 \mathrm{y}$ ). This may result in measurement sequences on sediments lasting sev-
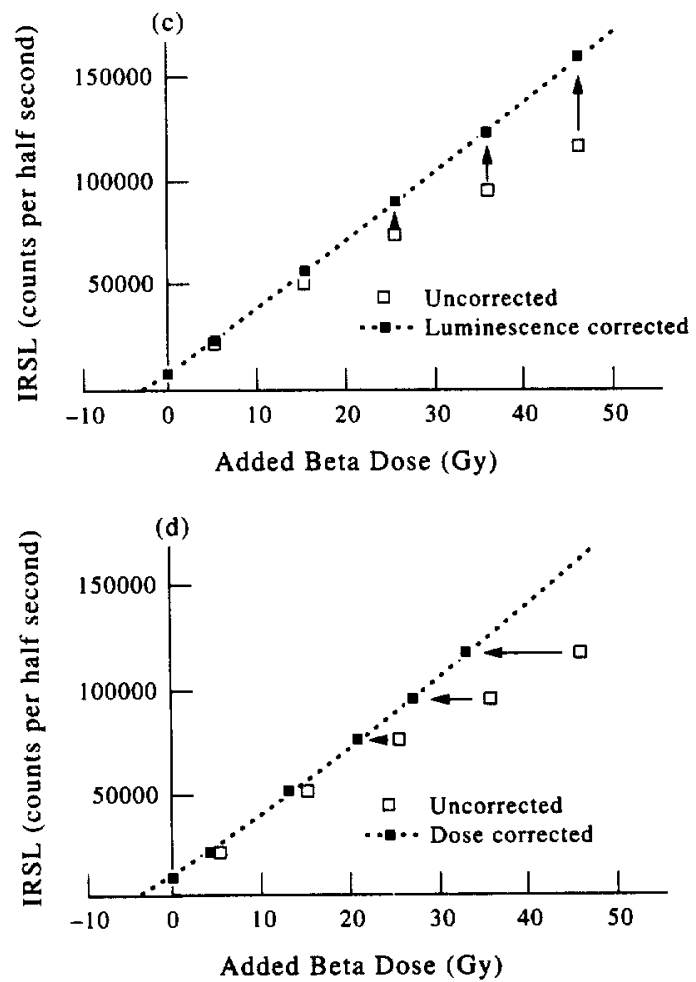

Fig. 24. Single aliquot additive dose data set for K-rich feldspars from a dune sand in New Zealand: (a) raw additive dose data, (b) preheat data set, (c) data from (a) corrected using data from (b) for the "luminescence correstion method" (Duller, 1991), and (d) as for (c) but using the "dose correction method" (Duller, 1994a) (from Duller, 1995b). 
eral weeks if irradiation is performed in a Risø $\mathrm{OSL} / \mathrm{TL}$ reader (e.g. Clarke et al., in preparation).

\subsection{Additive dose with luminescence correction}

The first automated "single aliquot" procedure was given by Duller (1991), although it should be mentioned that two discs are required in practice one for the additive dose growth curve and a second to determine the loss of signal caused by each successive preheat. In the initial study, potassium feldspar samples were subjected to a preheat, measure and irradiate cycle, which was repeated enough times to construct a meaningful growth curve which could be extrapolated to the dose axis. However, the growth curve which resulted from the $0.5 \mathrm{~s}$ IRSL measurements [Fig. 24(a)] could not be used in this way to obtain the $D_{\mathrm{E}}$ as each preheat caused depletion of the trapped electrons, greater than that caused by the IR exposure used to make the IRSL measurement. The growth curve thus needs to be corrected before the extrapolation is made. However, it should be noted that applications of the additive dose procedure make no allowance for initial non-linear growth, known as supra-linearity.

7.2.1. Separate preheat correction. The approach taken by Duller (1991) was to use additional discs (usually six, to improve precision, although one should be adequate if all the aliqucts are identical) and to subject them to a preheat and measurement cycle in which no additional dose is given. For the $\mathrm{K}$ feldspars a preheat of $10 \mathrm{~min}$ at $220^{\circ} \mathrm{C}$ repeated about 10 times reduces the IRSL to about $40 \%$ of the initial value [Fig. 24(b)]. The loss was not exponential.

Several correction procedures were attempted (Duller, 1991). To check that the procedure was appropriate, the sample disc used for the additive dose measurements was exposed to four additional cycles of preheat and measurement, but with no additional dose being given. The correction procedure was applied sequentially to all datcı points, including these. The ability of the procedure to give identical luminescence values for these points was the criterion used to select the best correction procedure. The best approach was that which treated each portion of the IRSL signal separately, with the natural IRSL being corrected one more time than the IRSL resulting from the first added dose, etc. [Fig. 24(c)]. This is interesting as it implies that each population of trapped charge is acting independently, a suggestion which is at odds with the notion of a single electron trap being responsible for the stable IRSL signal.

7.2.2. End-point preheat correcticn. A single aliquot procedure which indeed uses only a single disc was put forward by Galloway (1996). He used the RM 27/5-6-G decay curve obtained by even more preheat and measurement cycles at the end of the additive dose procedure to correct the additive dose growth curve on a disc-by-disc basis. The decay has been characterised by Galloway (1996) in terms of the number $(n)$ of preheat/measurement cycles employed and can be expressed as $\mathrm{f}(n)=1-\alpha \ln (n)$ where $\alpha$ is a constant. This correction procedure also required each portion of the IRSL to be corrected separately and had as its condition of acceptance the requirement for it to be applicable for the final data set, even after multiple correction. The procedure was tested by Galloway (1996) using Duller's published data set (Duller, 1991).

\subsection{Additive dose with dose correction}

When the additive dose growth curve deviates significantly from linearity, the correction in Section 7.2 is not appropriate. In this case the uncorrected luminescence growth curve is non-linear not just

(a)
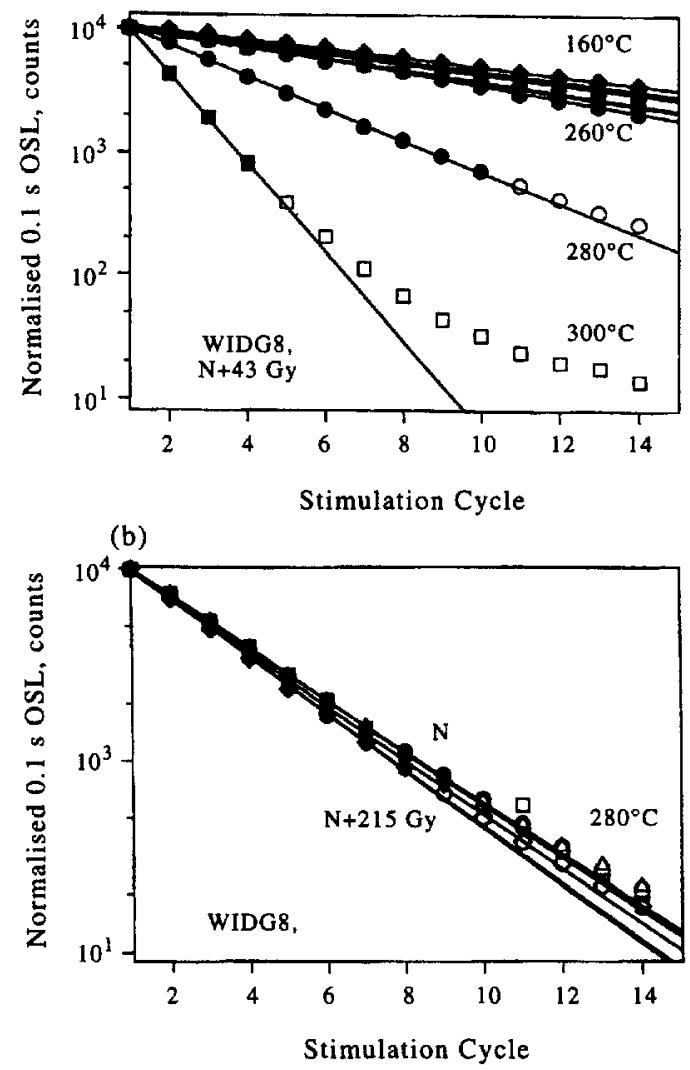

Fig. 25. Initial $\mathrm{OSL}\left(0.1 \mathrm{~s}\right.$ at $\left.110^{\circ} \mathrm{C}\right)$ measurements. (a) Pulsed decay curves for sample WIDG8 $(N+43 \mathrm{~Gy}$, $D_{\mathrm{E}}=58 \mathrm{~Gy}$ ) for $10 \mathrm{~s}$ repeated preheat cycles at $20^{\circ} \mathrm{C}$ temperature intervals from 160 to $300^{\circ} \mathrm{C}$. About $90 \%$ of the decay is exponential. (b) Pulsed decay curves for WIDG8 using a $280^{\circ} \mathrm{C} 10 \mathrm{~s}$ preheat when the sample had been given added doses of $0,1.7,3.4,6.8,13.5,27,54,108$ and $215 \mathrm{~Gy}$. The decay is independent of dose (from Murray et al., 1997). 
because of loss of signal owing to the preheat, but because of saturation. To deal with this Duller (1994a) proposed a "dose correction" procedure which applies the fractional loss with preheating to each dose component, rather than each luminescence component [Fig. 24(d)]. This procedure was used for $\mathrm{K}$ feldspars from dune sands dating back to $17.3 \mathrm{ka}$ (Clarke, 1994) and for even older fluvioglacial sands (Duller, 1994b).

\subsection{Single aliquots of quartz}

Following the success of the single aliquot additive dose procedure for feldspars, only a few studies (e.g. Stokes, 1994) wers reported in which the authors attempted a similar approach for quartz. An exception was a comprehensive study on some Australian aeolian sands (Murray et al., 1997) using broad-band green light stimulation. They observed that the initial $(0.1 \mathrm{~s})$ OSL signal from quartz decreased exponentially as a function of the number of times the sample was given a $10 \mathrm{~s}$ preheat. Figure 25(a) shows the decay in the OSL measured at $110^{\circ} \mathrm{C}$ (to keep the $100^{\circ} \mathrm{C}$ TL trap empty) for a sample (WIDG8, with $D_{\mathrm{E}}=58 \mathrm{~Gy}$ ) given an added dose of $43 \mathrm{~Gy}$. At least $90 \%$ of the decay of the OSL intensity is exponential when the charge is released optically (for temperatures below $260^{\circ} \mathrm{C}$ ) or by a combination of thermal and optical erosion (at above $260^{\circ} \mathrm{C}$ ). Further experiments demonstrated that this decay, as exemplified by the behaviour of the $10 \mathrm{~s} 280^{\circ} \mathrm{C}$ preheats, was independent of the applied dose [Fig. 25(b)].

Based on the data in Fig. 25, and similar data for other Australian sedimertary quartz, Murray et al. (1997) formulated a simple model to describe the decay correction procedure to be applied to similar samples in an additive dose single aliquot protocol. It uses the data points obtained at the end of the additive dose cycle, when repeated preheat/stimulation cycles are performed with no added dose, to determine the decay constant; this is then applied to the additive dose data set in a correction procedure analogous to the "luminescence correction" procedure of Duller (1991), discussed in Section 7.2. This approach is also similar to that of Galloway (1996) in that it is a truly single aliquot procedure, but differs fundamentally in that the decay per cycle is constant; Galloway's empirical relationship for feldspars (given in Section 7.2.2) is necessary because the decay rate decreases with measurement cycle, as found in earlier measurements by Duller (1991). Murray et al. (1.997) further demonstrated the strength of this protocol by applying it to a number of aliquots, using different $10 \mathrm{~s}$ preheats from 150 to $300^{\circ} \mathrm{C}$, in order to produce a plateau of $D_{\mathrm{E}}$ against preheat temperature (see Section 7.8).

Stokes (1994) also investigated whether dosedependent sensitivity changes would occur as a result of the sequence used for the single aliquot additive dose method when used for quartz. No such change occurred using a preheat of $160^{\circ} \mathrm{C}$ for $16 \mathrm{~h}$ and he obtained a date on an Australian sand using this procedure, but not carried out in an automated reader. Liritzis et al. (1994) also used an additive dose single aliquot protocol for quartz extracted from pottery, but using a green diode array (Galloway, 1994), rather than a laser or green light source.

\subsection{Regeneration}

Conceptually the regeneration method is the simplest approach of single aliquot dating. For OSL it is assumed that the signal at deposition (if any) is that which is reached by the laboratory bleaching. It is assumed that the laboratory bleaching does not alter the OSL sensitivity as measured with the regenerated signal. The sample can then be irradiated and measured in a repetitive cycle, using different radiation doses to construct a growth curve. Duller (1991) tested this approach for IRSL from potassium feldspars from sediments and used a 10 -min preheat at $220^{\circ} \mathrm{C}$ to remove unstable components in the signal. Using an IR bleach to zero the IRSL, he found a progressive change in sensitivity (of the order of $5-10 \%$ ) as repeat measurements were made. Richardson (1994) found a range of sensitivity changes when the IRSL of potassium feldspars was measured for recent dune sands which had been exposed to a variety of natural and artificial light sources, and the behaviour agrees well with predictions from a recent modelling study by McKeever et al. (1997). The amount of sensitivity change for IRSL signals appears to relate to the bleaching history of the grains, as found for studies on colluvial samples ( $\mathrm{Li}$ and Wintle, 1991, 1992; Wintle et al., 1995a,b). They suggest that, for grains which have had their IRSL, but not their TL, zeroed at deposition, IR bleaching in the single aliquot regeneration method gives reliable $D_{\mathrm{E}}$ values for potassium feldspars.

Tso and $\mathrm{Li}$ (1994) used a single aliquot regeneration procedure for fine grains from pottery, with heating rather than bleaching being used as the more appropriate zeroing procedure; their procedure actually required three discs.

The single aliquot regeneration method has also been attempted for quartz, but sensitivity changes have been reported which precluded this simple method being adopted (Mejdahl and Bøtter-Jensen, 1994). Since then Murray and Roberts (submitted) have described a single aliquot regeneration procedure for quartz which explicitly measures the sensitivity change resulting from each preheat/bleach cycle, using the $110^{\circ} \mathrm{C}$ TL peak response (as described in Section 7.7). They showed that the $D_{\mathrm{E}}$ observed on one aeolian sample using this approach 
(a)

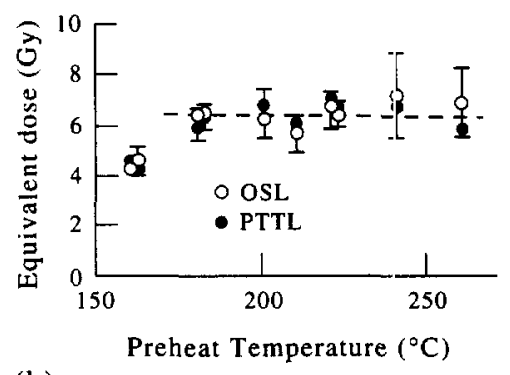

(b)
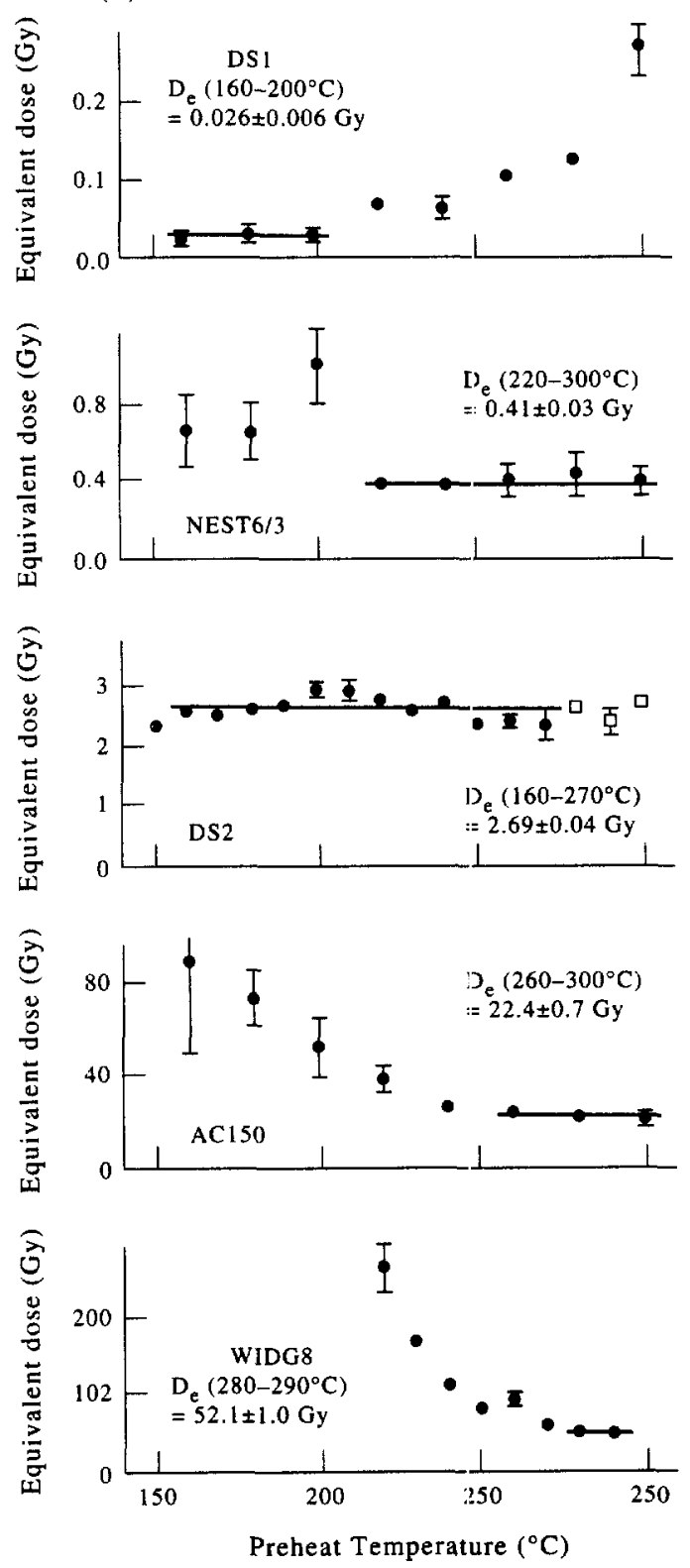

Fig. 26. Values of $D_{\mathrm{E}}$ obtained using si.ngle aliquot additive dose procedures for quartz grains given as a function of the temperature of the $10 \mathrm{~s}$ preheat employed. (a) Values of $D_{\mathrm{E}}$ obtained using a modified SARA procedure (from Murray, 1996a), (b) values of $D_{\mathrm{E}}$ obtained using a simpler additive dose procedure (from Murray et al., 1997) showing that older samples (with larger values of $D_{\mathrm{E}}$ ) require a higher preheat temperature. was in excellent agreement with that expected from the known $\left({ }^{14} \mathrm{C}\right)$ age, and with the observed values using TL, multiple aliquot OSL additive dose, and single aliquot OSL additive dose protocols. This new procedure has also been applied to many other Australian sediments, and to sediments and heated quartz samples from Scandinavia (Andrew Murray, personal communication).

\section{6. $D_{E}$ versus preheat temperature}

The modified SARA method (described in Section 5.6) was also applied to the integrated phototransferred TL (PTTL) measured in the $110^{\circ} \mathrm{TL}$ peak after the green light exposure (required in the OSL study). The value of $D_{\mathrm{E}}$ obtained for both the OSL and the PTTL study were shown to be independent of the $10 \mathrm{~s}$ preheat used, provided that the preheat temperature was above $180^{\circ} \mathrm{C}$ [Fig. 26(a)].

In Section 6, the importance and necessity of employing a preheat prior to the OSL measurement was discussed. For single aliquot procedures a preheat is just as important, but the effect it has is more difficult to monitor. Using the modified SARA protocol, Murray (1996a) selected a preheat of $10 \mathrm{~s}$ at $190^{\circ} \mathrm{C}$ for routine use for young fluvial sediments, based on Fig. 26(a).

In Section 7.4, the additive dose single aliquot procedure of Murray et al. (1997) was explored. The protocol was applied using $10 \mathrm{~s}$ preheats at 10 or $20^{\circ} \mathrm{C}$ intervals between 150 and $300^{\circ} \mathrm{C}$. Results were obtained on 5 samples which had values of $D_{\mathrm{E}}$ ranging from $0.026 \mathrm{~Gy}$ up to $52 \mathrm{~Gy}$ [Fig. 26(b)]. The older samples showed a tendency for $D_{\mathrm{E}}$ to give a plateau at the higher preheat temperatures (above $260^{\circ} \mathrm{C}$ ). Using the values in the plateau region gave excellent agreement of the value $D_{\mathrm{E}}$ with that derived from multiple aliquot additive dose procedures. For one of these older samples (a 10000 -year-old aeolian sand from Allen's Cave in South Australia with $D_{\mathrm{E}}$ around $24 \mathrm{~Gy}$ ) a preheat of $10 \mathrm{~s}$ at $280^{\circ} \mathrm{C}$ was chosen, when a single grain additive dose protocol was applied (Murray and Roberts, in press).

In a study on a slightly older $(30000 \mathrm{y}$ and $\left.D_{\mathrm{E}} \sim 58 \mathrm{~Gy}\right)$ sand from Widgingarri in western Australia, Wintle and Murray (submitted) showed that for a simple single aliquot regeneration dating procedure, the value of $D_{\mathrm{E}}$ obtained was highly dependent on the preheat temperature. In their experiments they compared the natural OSL with that for a sample which had been bleached and given a regeneration dose of $56 \mathrm{~Gy}$. The results were obtained using a single disc to which successive pulse anneal preheats for $10 \mathrm{~s}$ were applied between each $0.1 \mathrm{~s}$ measurement of its $\mathrm{OSL}$ at $125^{\circ} \mathrm{C}$, first for the natural dose and then for the regenerated dose. Within this procedure a small test dose was 
applied and the $110^{\circ} \mathrm{C}$ TL peak was measured to monitor for sensitivity changes resulting from the preheat immediately prior : 0 the delivery of the test dose. This showed a thermal activation process which was significantly different for the natural and laboratory irradiations. This has led to the development of a new measurement protocol for single aliquots of sediments (Murray and Roberts, submitted) and fired grains (Murray and Mejdahl, submitted).

\subsection{Single-grain procedures}

The importance of single-grain TL measurements has been demonstrated both for quartz (Huntley et

(a)

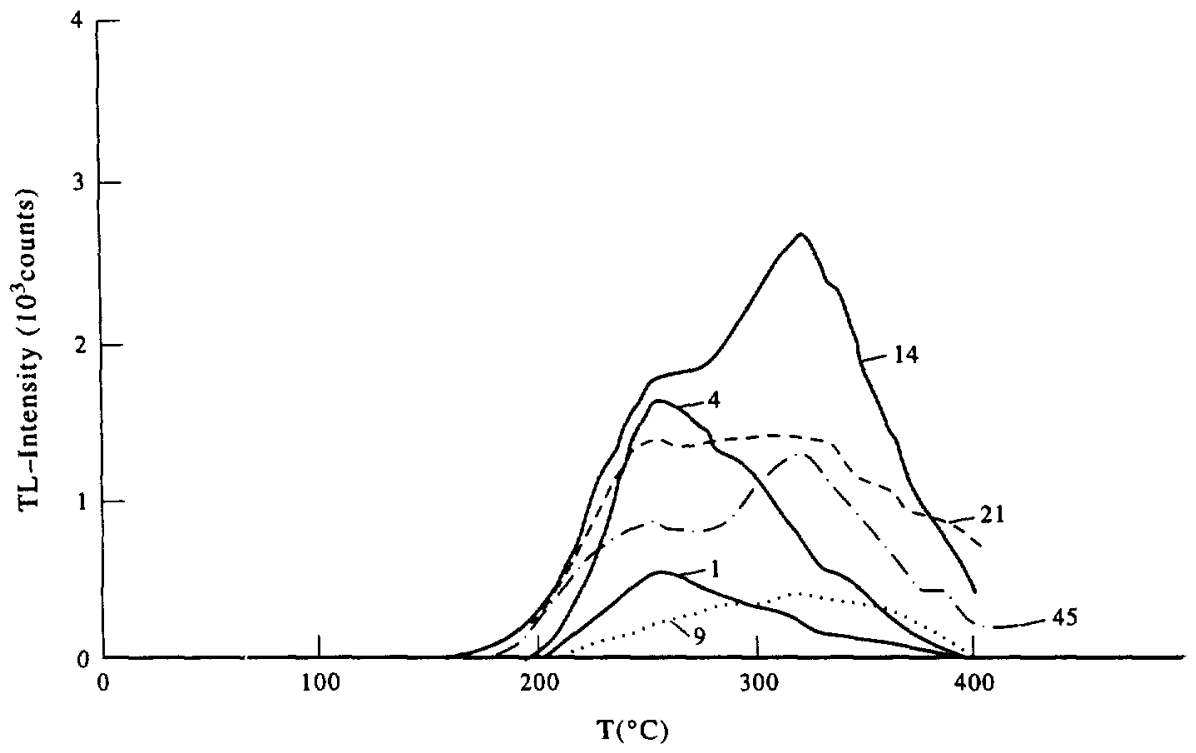

(b)

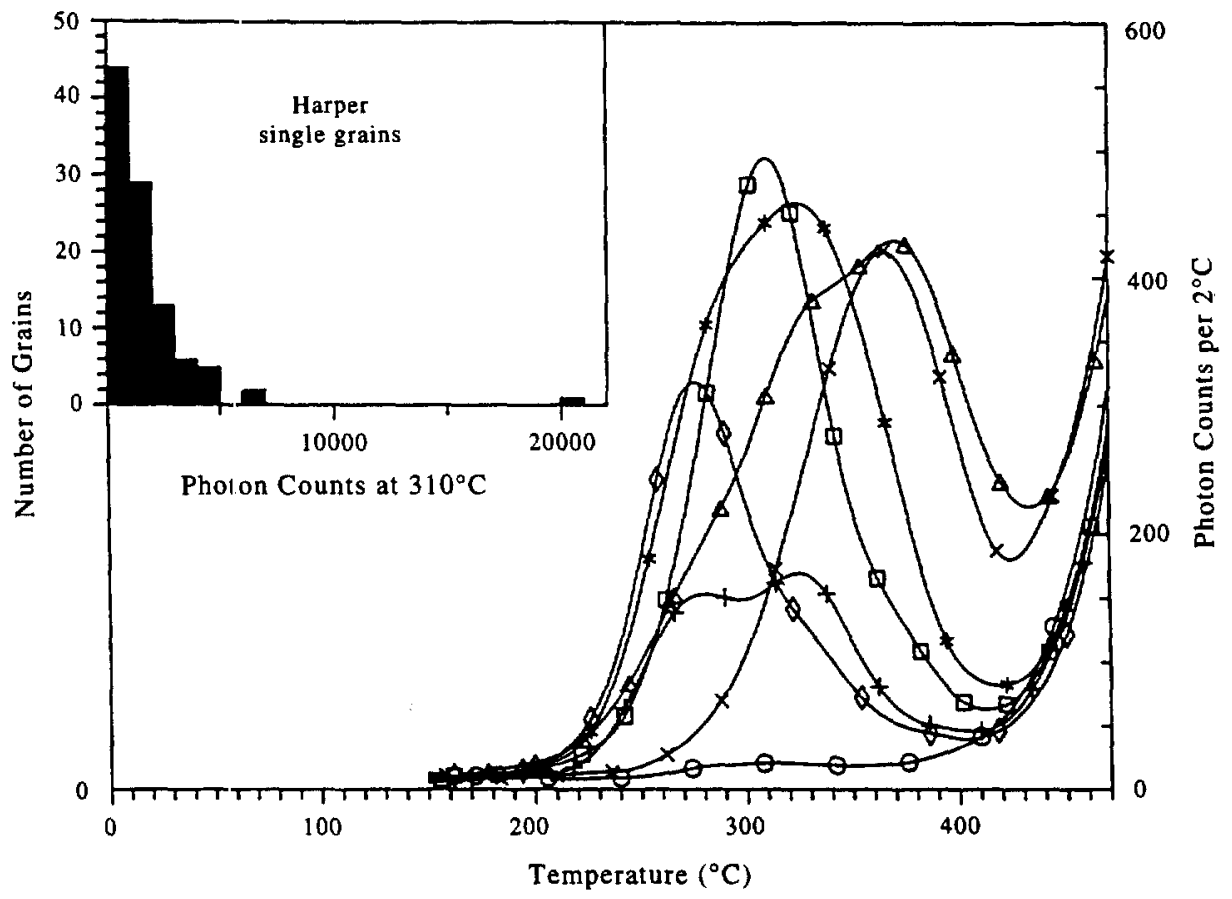

Fig. 27. (a) Glow curves for 6 individual grains of feldspar from Danish cover sands (from Grün et al., 1989). (b) Glow curves for 7 individual $150-180 \mu \mathrm{m}$ grains of quartz from an Australian dune, and the inset shows the distribution in brightness (photon counts between 300 and $320^{\circ} \mathrm{C}$ (from Huntley et al., 1993b). 
al., 1993b) and $\mathrm{K}$ feldspars (Grün et al., 1989). Both studies showed the variability in both intensity and glow curve shape [Fig. 27(a,b)] and led to the conclusion that the light was comirg from a very small number of grains. In the study of the Australian dune sands, this variation in natural light level is best explained by the different grains having different sensitivities, or possibly different saturation levels, since the dunes a:e several hundred thousand years old.

In the case of material which may incorporate unbleached grains, the ability to measure the luminescence signal from single grains may permit clear distinction between relatively recently deposited well-bleached grains and unbleached grains incorporated by reworking of older sedirnents. Lamothe et al. (1994) were particularly suczessful in their results for late glacial marine sand: from Québec. They found a range of natural IRSI, signal intensities from individual grains picked from the 500$1000 \mu \mathrm{m}$ grain size range, which were subsequently shown to have an internal $\mathrm{K}$ content of $11.7 \%$. Using the additive dose single aliquot procedure (with luminescence correction) they obtained $D_{\mathrm{E}}$ and then calculated an age for each grain, and the results are shown in Fig. 28. Half the grains are too old and it is suggested that these might have been derived from older non-glacial sediments. The others are about $30 \%$ too young and it is suggested that these might exhibit anomalous fading. The combined age obtained, as would have been obtained if all the grains had been on one disc and used in a multiple-grain single aliquot measurement, was about $51 \mathrm{ka}$, similar to actual measurements reported elsewhere (Balescu and Lamothe, 1994).

Lamothe and Auclair (1997) have attempted to develop a laboratory test to identify the presence of unbleached grains in 6 samples for which the multiple aliquot additive dose procedure for IRSL gave ages far in excess of the radiocarbon or stratigraphic age estimate. They hand picked up to 70 single K-feldspar grains of $500 \mu \mathrm{m}$ to $1 \mathrm{~mm}$ diameter and compared the natural IRSL with that for the IRSL obtained after giving an additional gamma dose to the same grain. The ratios $R_{\mathrm{I}}$ are shown in Fig. 29 alongside the multiple aliquot data set. The results for CES2 demonstrate the homogeneity of the grains in that sediment, suggesting that none of the grains in the fluvioglacial sand were exposed to light. The other two samples gave a wider range of $R_{\mathrm{I}}$ suggesting that these samples have a significant proportion of unbleached (or perhaps poorly bleached) grains. Fading tests were also carried out on the same single grains from CES3, chosen to see if there was significant fading in grains from the Saint Césaire site. Some grains lost almost half of their IRSL in the 7-month room-temperature storage, even though they were preheated at $220^{\circ} \mathrm{C}$ for $10 \mathrm{~min}$. As a result of this study, Lamothe and Auclair (1997) proposed a sequence of measurements to be performed on 50 grains to assess the homogeneity of bleaching at the time of burial and the presence of anomalous fading.

Based on the possibility of using the $110^{\circ} \mathrm{C}$ TL peak to monitor the effect of sensitivity changes in

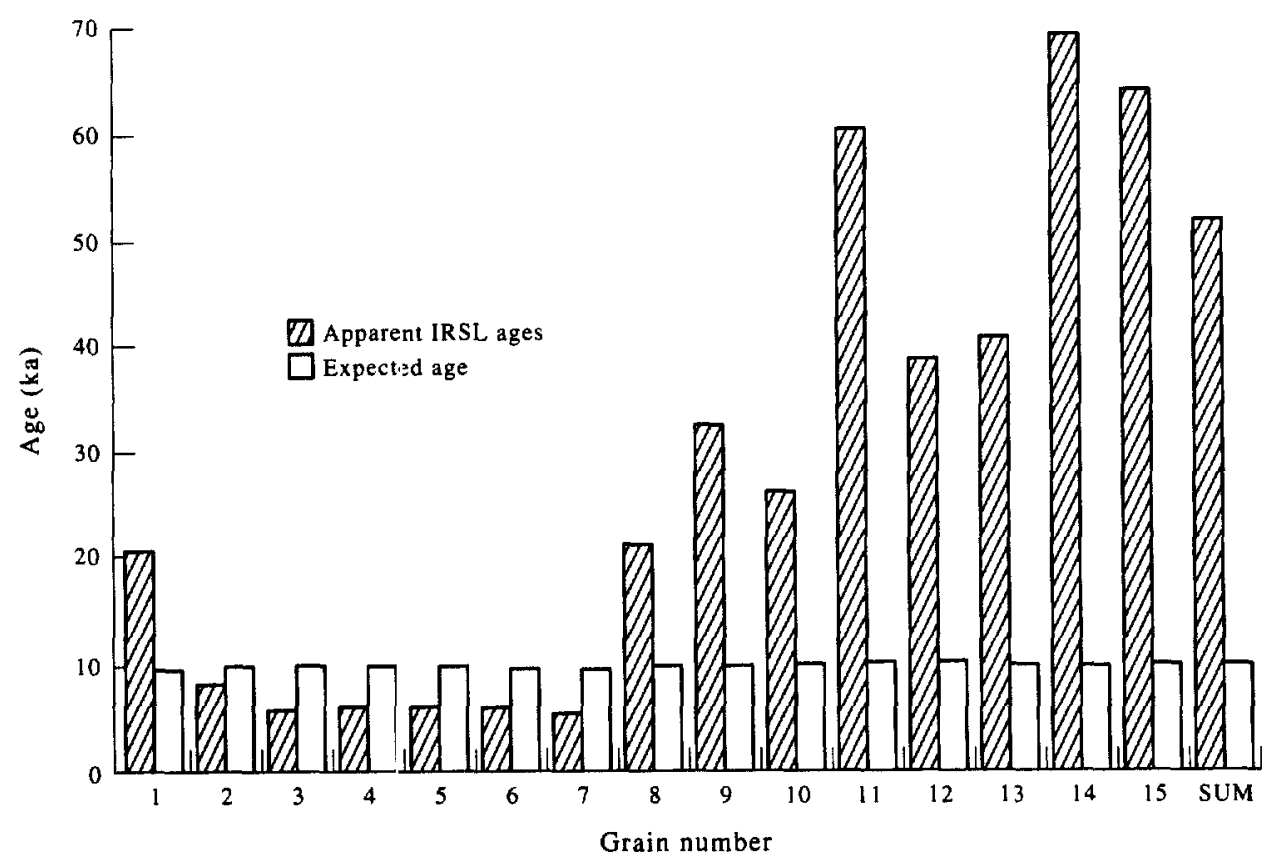

Fig. 28. Calculated ages baseci on the IRSL from 15 individual K-rich feldspars $(500-1000 \mu \mathrm{m})$ compared with the age of $9.5 \mathrm{ka}$ expected on the basis of the geological context and related radiocarbon date (from Lamothe et al., 1994). 

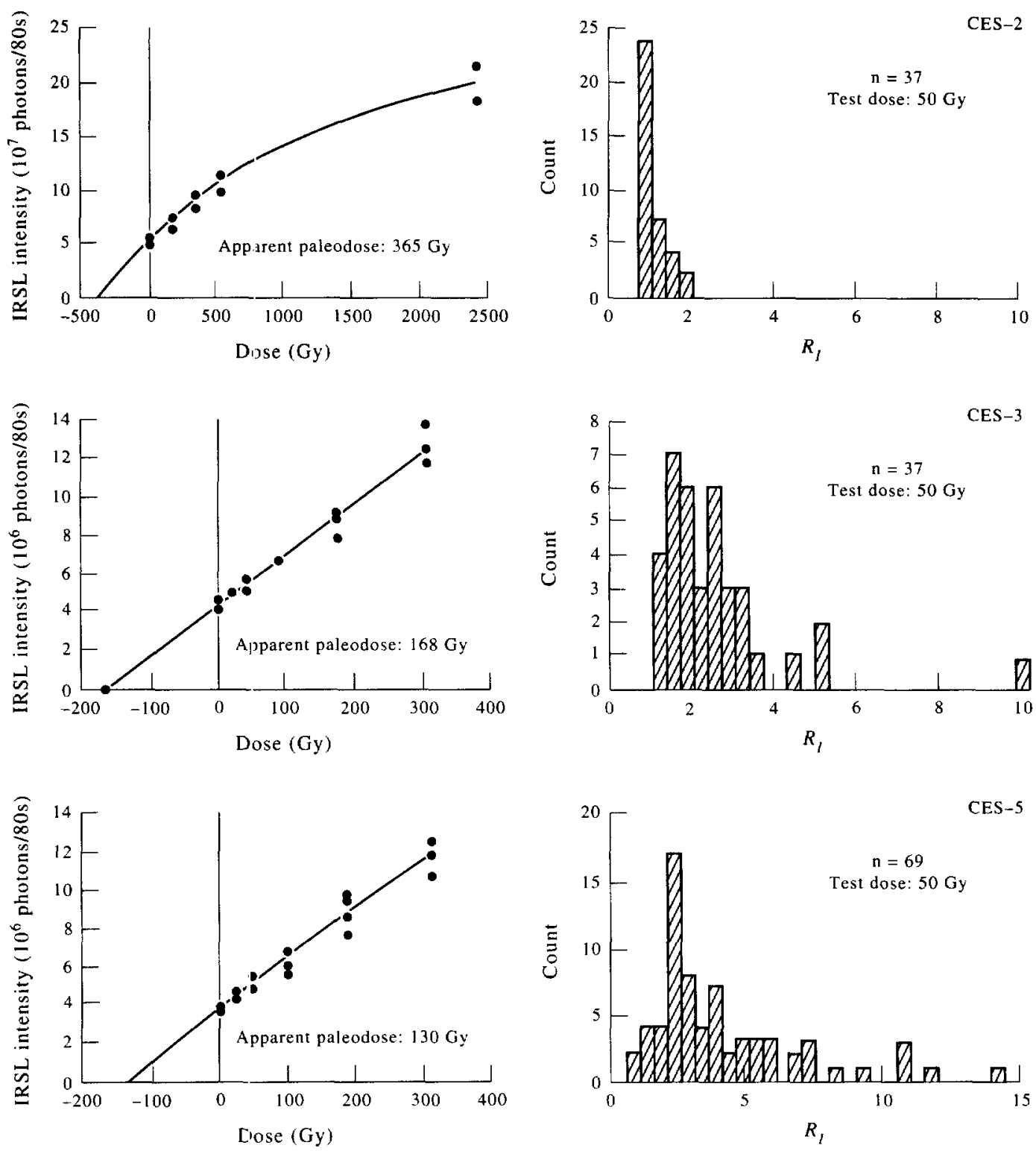

Fig. 29. IRSL growlh curves using a conventional multiple aliquot data set for $150-250 \mu \mathrm{m}$ K-rich feldspars for three samples. Also shown are the ratios, $R_{\mathrm{I}}$, of the natural IRSL to that from a 50 Gy dose for single grains of $500 \mu \mathrm{m}$ to $1 \mathrm{~mm}$ diameter from the same three sediments.

natural and regenerated OSL signals from quartz (Wintle and Murray, 1997), Murray and Roberts (submitted) proposed a single aliquot regeneration protocol. This was successfully applied to single grains of quartz extracted from a sand from an Australian cave site (see Section 7.5) (Murray and Roberts, in press). Regeneration single aliquot measurements on 25 grains showed a wide range of response, both in terms of brightness and growth curve. The histogram of values of $D_{\mathrm{E}}$ gave a standard deviation of $5 \mathrm{~Gy}$, which it was concluded was the result of non-uniformity in the beta radiation field in the environmen: (Olley et al., 1997). The average value was in agreement with the best esti- mates of $D_{\mathrm{E}}$ obtained using single and multiple protocols for aliquots containing a large number of grains (Murray and Roberts, in press).

\subsection{Multiple evaluations of $D_{E}$}

The application of the single aliquot procedures presented in the preceding sections to a number of aliquots enables additional information to be obtained concerning the uniformity of the sedimentary deposit, both with regard to completeness of bleaching and microdosimetry. Such an approach assists in the production of a precise and accurate 
age. Typically, around 20 determinations are made. Li (1994) plotted individual values of $D_{\mathrm{E}}$ against natural intensity, $I_{\mathrm{n}}$, for each disc of feldspars taken from several samples of colluvium. The distribution of these data points, each representing a unique value of $D_{\mathrm{E}}$ and $I_{\mathrm{n}}$, were interpreted in terms of varying sensitivity of the grains and/or varying values of dose recorded by the grains. The latter would result from incomplete bleaching of the sediment. By reducing the number of grains of potassium feldspar on a disc from a few thousand to only a few hundred, Li (1994) showed that for the two youngest samples he was able to obtain the smallest values of $D_{\mathrm{E}}$ for discs with relatively few grains. $\mathrm{He}$ concluded that these values were likely to be closest to the value of $D_{E}$ representing the age of the deposit.

Similar plots for modern colluvial and alluvial sediments have been obtained by Porat et al. (1997). Figure 30(a) shows that for the potassiumrich feldspars from the colluvium, the data fall on a line through the origin, suggesting that the grains

(a)

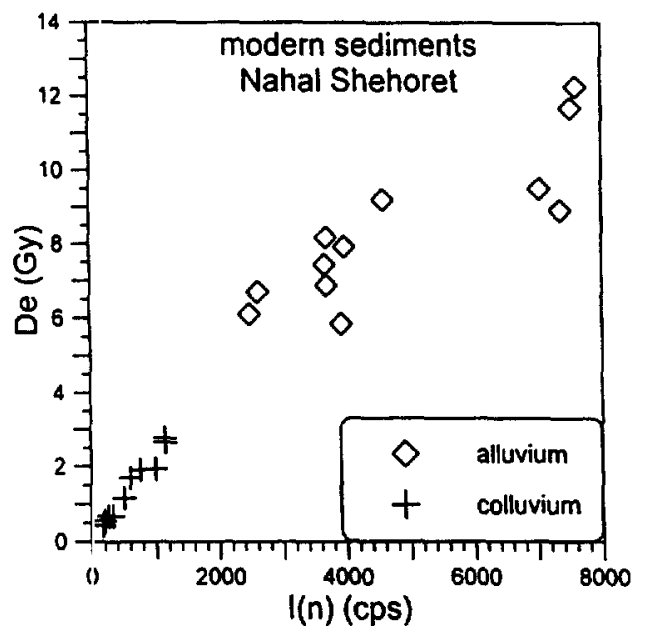

(b)
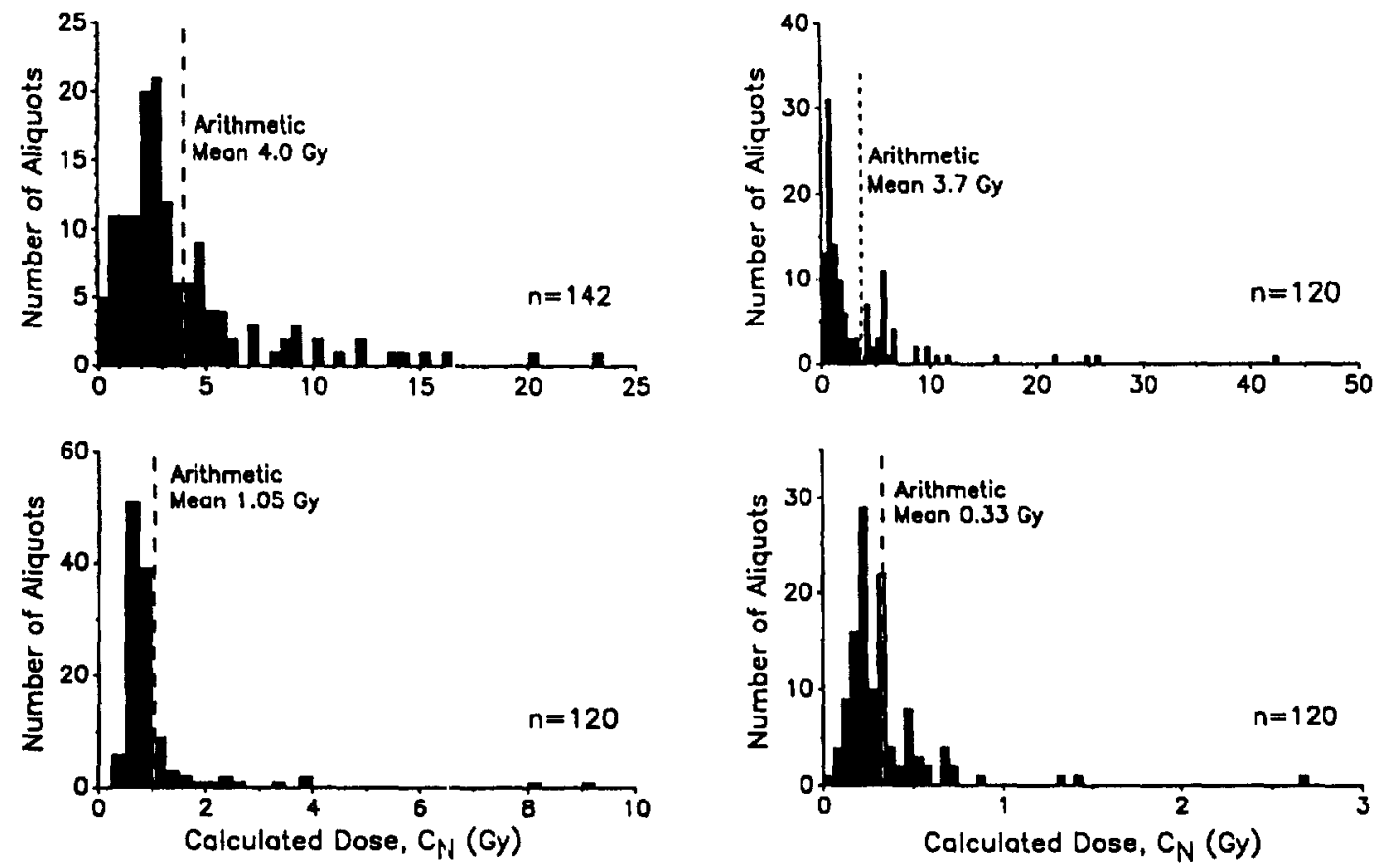

Fig. 30. (a) $D_{\mathrm{E}}$ plotted agains1 $I_{\mathrm{n}}$ (natural IRSL intensity) for single aliquot measurements of two modern sediments, an alluvium and a colluvium from southern Israel. The sediments are not completely bleached, but the colluvium is better bleached (from Porat et al., 1997). (b) Histograms of single aliquot $D_{\mathrm{E}}$ values obtained for $1 \mathrm{mg}$ samples of $90-250 \mu \mathrm{m}$ quartz from 4 modern water-lain deposits from Australia (from Murray et al., 1995). 
all have the same IRSL sensitivity, but have been bleached to different extents. The samples of alluvial origin appear to be less well bleached. The values of $D_{\mathbf{E}}$ for the two samples result in "ages" of $540 \pm 375 \mathrm{y}$ and a range of $2300-5100 \mathrm{y}$, respectively. These represent small age offsets compared with the ages of the fault-related sediments which were the focus of the study

Clarke (1996) has taken this idea further by plotting normalised values of $D_{\mathbf{E}}$ versus normalised values of $I_{n}$ and has developed criteria based on the scatter of the $D_{\mathrm{E}}$ data set. These criteria for level of bleaching at deposition were discussed using examples of potassium-rich feldspars from wellbleached aeolian deposits and incompletely. bleached colluvial and fluvial deposits.

Murray et al. (1995) used another approach for their single aliquot data on quartz from modern water-lain deposits from Australia. They made 120 measurements of $D_{\mathrm{E}}$ using discs with only $1 \mathrm{mg}$ of sample, containing about 130 grains. They plotted them as histograms [Fig. 30(b)] and observed a tight cluster of values near the origin, with a few discs with significantly higher values. As expected, the distribution has a rapidly rising edge on the low dose side. They proposed that this edge can be used to define the depositional age for poorly bleached samples. This procedure was further tested on a modern dune sand and a modern fluvial sediment from a bar and then applied to a short core taken within the flood channel of the Namoi River, New South Wales (Olley et al., in press). Using aliquots consisting of 60-100 grain;, asymmetric histograms of values of $D_{\mathrm{E}}$ were obtained, suggesting that the aliquots were poorly bleached. Age estimates ranging from $22 \mathrm{y}$ at the top of the core to $146 \mathrm{y}$ at the bottom were obtained by aking the mean value of the lowest $5 \%$ of $D_{\mathrm{E}}$ values for each of the sample data sets.

\section{COMBINED OSL/TL MEASUREMENTS}

\subsection{Quartz TL, feldspar Ti, and feldspar IRSL}

From the preceding sections, it can be seen that there are a number of luminescence signals which are reduced at different rates when exposed to sunlight. Using more than one of these signals for grains from the same sedimentary unit may enable an assessment to be made of the extent to which the grains were exposed to light at deposition. This may be carried out using different minerals, e.g. using the quartz TL and feldspar TL and IRSL as employed by Rendell et al. (1994a). They studied sand ramps in the Mojave Desert in California. These are formed as sand is blown up against the base of a mountain range; the sediments are made up primarily of grains of aeolian origin, as well as some which will have moved down slope in occasional heavy rain storms. In this situation it might be expected that the colluvial grains will have had a shorter light exposure, and thus the age obtained using the more rapidly bleached signal, namely the feldspar IRSL, will be closest to the true age. Since the feldspar and quartz grains would have had different dose rates, owing to the internal potassium content of the potassium-rich feldspar separate, the ages, rather than $D_{\mathrm{E}}$ values, were compared for each signal. For the younger samples, up to $20 \mathrm{ka}$, all three signals are in good agreement, suggesting that the units that were sampled were comprised of well-bleached grains.

\subsection{The RBP, SBP and $O S L$ from quartz}

In a study of luminescence signals from grains extracted from an excavated rock shelter in Botswana, Feathers (1997) used three signals from quartz, namely the RBP TL (Section 2.1.2), the SBP TL (Section 2.1.1) and the OSL. There was concern that the sand units, which were primarily aeolian, may have contained grains which had been either washed in or derived from the walls of the rock shelter. For the three youngest samples, the values of $D_{\mathrm{E}}$ obtained for the SBP and for the OSL single aliquot data sets showed agreement, implying that the samples were well bleached at deposition. The RBP measurements lacked precision and this resulted in large errors in the $D_{\mathrm{E}}$ values obtained, thus precluding any useful conclusion being drawn.

Another rock shelter site where roof-fall material may have contributed to the deposits is Puritjarra in central Australia (Smith et al., 1997). Samples from this site have played a major role in the development of TL techniques for quartz (Prescott and Fox, 1990). At this site more than 30 radiocarbon dates on charcoal were available for comparison with the TL results. Data for TL growth curves were obtained using both the total bleach technique and the selective bleach method, and the two data sets were each fitted using the "Australian slide" procedure; the growth curves were fitted using a combination of a saturating exponential and a linear curve. The total bleach and the selective bleach methods give very similar values of $D_{\mathrm{E}}$, and these are in agreement with the limited number of OSL ages which were also presented in the paper. In addition, experiments were carried out to see if the clay rinds on the grains might have prevented total bleaching of the RBP at deposition, but it was concluded that this was not the case. However, the ages obtained using this peak were significantly larger than the associated radiocarbon dates, by $6000-$ $10000 \mathrm{y}$. Some of the discrepancy could be explained by sampling problems, but the more likely explanation is that some unbleached grains derived from the sandstone bedrock were incorporated in the sediments without exposure to light, and this is discussed in detail by Smith et al. (1997). 
This problem could be explored using single grain OSL procedures as used at Ngarratullgan Cave, a rock shelter in Queensland (David $t$ t al., 1997). In this case the single grain results were in agreement with the multiple aliquot measurement, indicating that it was unlikely that any decomposed bedrock was incorporated in the sediment.

\subsection{TL and IRSL from feldspars}

Balescu et al. (1997) extracted alkali feldspars (containing $7 \% \mathrm{~K}$ ) from two interglacial estuarine sediments, assigned to oxygen isotope stages 9 (303-339 ka) and 7 (186-245 ka). Following at least a year's storage after irradiation ard a subsequent preheat at $160^{\circ} \mathrm{C}$ for $16 \mathrm{~h}$, the additive dose TL and IRSL growth curves gave identica! values of $D_{\mathrm{E}}$, with agreement also being found for a Pliocene (3$4 \mathrm{Ma}$ ) sand being used to assess the degree of age underestimation (see Section 2.3). The luminescence ages that were calculated using the measured dose rate and values of $D_{\mathbf{E}}$ indicated significant age underestimation for the interglacial deposits, thus suggesting that long-term fading afects equally the TL and IRSL signals for these feldspars from north-western France. Using the Pliocene sample, Balescu et al. (1997) suggest that for these alkali feldspars, the mean lives for fading in the environment of north-western France, experiencing both interglacial and glacial temperatures for tens of thousands of years, are close to 1 million years. Using equations which allow for both non-linear luminescence growth and thermal ading (Mejdahl, 1988a, 1989), both the IRSL and J $L$ data result in corrected ages in excellent agreemerit with the stratigraphic evidence. This agreement suggests that the estuarine grains were well bleached when originally deposited.

\section{CONCLUSIONiร}

The variety of luminescence signals that can be obtained from the most common sedimentary minerals, quartz and feldspars, provide useful dating tools for Quaternary sediments.

Each signal, whether TL, OSL cr IRSL, has particular advantages and disadvantages with regard to long-term thermal stability, intrinsic sensitivity and optical bleachability.

New developments, such as single aliquot procedures, permit a better understanding of the efficiency of the bleaching process at deposition, as well as providing a less time-corsuming measurement procedure.

Fundamental experiments on the sensitivity of the luminescence signals to light and heat play a fundamental role in the developinent of the most appropriate laboratory protocols for use in routine dating applications. Such studies; have been per- formed on quartz, but similar studies are required for feldspars.

The most exciting developments in the past 7 years have been the OSL and IRSL methods based on single aliquot approaches. For quartz these have been used to obtain dates for single grains for samples with ages of several tens of thousand of years. For younger samples, a few hundred grains are required to obtain a statistically meaningful signal. These results have been obtained primarily for Australian quartz and it will be interesting to see results obtained from other parts of the world.

Acknowledgements-I wish to thank Geoff Duller and Andrew Murray for critical comments on this paper at various stages of its production, John Vogel and Gill Collett for the data in Fig. 11, and Rita Howcroft for typing the first draft, whilst the author was on study leave at QUADRU, CSIR, Pretoria, South Africa. Fundamental research in luminescence dating in the Aberystwyth Luminescence Laboratory is currently funded by NERC grant GST/02,0762

\section{REFERENCES}

Aitken, M. J. (1984) Non-linear growth: allowance for alpha particle contribution. Ancient TL 2, 2-5.

Aitken, M. J. (1985) Thermoluminescence Dating. Academic Press, London.

Aitken, M. J. (1992) Optical dating. Quaternary Science Reviews 11, 127-131.

Aitken, M. J. and Bussell, G. D. (1979) Zero-glow monitoring (ZGM). Ancient TL 6, 13-15.

Aitken, M. J. and Smith. B. W. (1988) Optical dating: recuperation after bleaching. Quaternary Science Reviews 7, 387-393.

Aitken, M. J. and Xie, J. (1992) Optical dating using infrared diodes: young samples. Quaternary Science Reviens 11, 147-152.

Bailiff, I. K. (1993) Measurement of the stimulation spectrum $(1.2-1.7 \mathrm{eV})$ for a specimen of potassium feldspar using a tunable solid state laser. Radiation Protection Dosimetry 47, 649-653.

Bailiff, I. K. (1994) The pre-dose technique. Radiation Measurements 23, 471-479.

Bailiff, I. K. and Barnett, S. M. (1994) Characteristics of infrared-stimulated luminescence from a feldspar at low temperatures. Radiation Measurements 23, 541 545

Bailiff, I. K. and Poolton, N. R. J. (1991) Studies of charge transfer mechanisms in feldspars. Nuclear Tracks and Radiation Measurements 18, 111-118.

Balescu, S. and Lamothe, M. (1994) Comparison of TL and IRSL age estimates of feidspar coarse grains from waterlain sediments. Quaternary Geochronology (Quaternary Science Review's) 13, 437-444.

Balescu, S., Dupuis, C. and Quinif, Y. (1988) TL stratigraphy of pre-Weichselian loess from NW Europe using feldspar coarse grains. Quaternary Science Reviewis 7, 309-313.

Balescu, S., Lamothe, M. and Lautridou, J.-P. (1997) Luminescence evidence for two Middle Pleistocene interglacial events at Tourville, northwestern France. Boreas 26, 61-72.

Baray, M. M. and Zöller, L. (1994) Methodological aspects of thermoluminescence dating of Late Glacial and Holocene dune sands from Brandenburg, Germany. Quaternary Geochronology (Quaternary Science' Reviews) 13, 477-480. 
Barnett, S. M. and Bailiff, I. K. (1997) Infrared stimulation spectra of sediments containing feldspars. Radiation Measurements 27, 237-242.

Bell, W. T. and Zimmerman, D. W. (1978) The effect of HF etching on the morphology of quartz inclusions for thermoluminescence dating. Archaeometry 20,6365.

Berger, G. W. (1990) Effectiveness of natural zeroing of the thermoluminescence in sediments. Journal of Geophysical Research 95(B8), 12375-12397.

Berger, G. W. (1995) Progress in luminescence dating methods for Quaternary sediments. In: Dating Methods for Quaternary Deposits (eds Rutter, N. W. and Catto, N. R.), pp. 81-104. Geological Association of Canada.

Berger, G. W. (1996) Comment on paper by Guibert et al.. Ancient TL 14(3), 15-16.

Berger, G. W. and Anderson, P. M. (1994) Thermoluminescence dating of an Arctic lake core from Alaska. Quaternary Geochronology (Quaternary Science Reviews) 13, 497-501.

Berger, G. W. and Luternauer, J. J. (1987) Preliminary fieldwork for thermolum nescence dating studies at the Fraser River delta, British Columbia. Geological Survey of Canada Paper 87-1A, pp. 901-904.

Berger, G. W., Pillans, B. J. and Palmer, A. S. (1992) Dating loess up to $800 \mathrm{ka}$ by thermoluminescence. Geology 20, 403-406.

Bøtter-Jensen, L. (1997) Lumiaescence techniques: instrumentation and methods. Radiation Measurements 27, 749-768.

Bøtter-Jensen, L. and Duller, 1G. A. T. (1992) A new system for measuring optically stimulated luminescence from quartz samples. Nuclear Tracks and Radiation Measurements 20, 549-55.3.

Bøtter-Jensen, L., Duller, G. A. T. and Poolton, N. R. J. (1994) Excitation and emission spectrometry of stimulated luminescence from quartz and feldspars. Radiation Measurements 23, 613-616.

Chawla, S., Dhir, R. P. and Singhvi, A. K. (1992) Thermoluminescence chronology of sand profiles in the Thar Desert and their implications. Quaternary Science Review's 11, 25-3:

Clark, P. A. and Templer, R. H. (1988) Thermoluminescence dating of materials which exhibit anomalous fading. Arrhaeometry 30, 19-36.

Clarke, M. L. (1994) Infra-red stimulated luminescence ages from aeolian sand and alluvial fan deposits from the Eastern Mojave desert, California. Quaternary Geochronology (Quaternary Science Reviews) 13, 533-538.

Clarke, M. L. (1996) IRSL dating of sands:bleaching characteristics at deposition inferred from the use of single aliquots. Radiation Measurements 26, 611-620.

Clarke, M. L. and Rendell, H. M. (1997a) Stability of the IRSL spectra of alkali feldspars. Physica Status Solidi (b) 199, 597-604.

Clarke, M. L. and Rendell, H. M. (1997b) Infra-red stimulated luminescence spectra of alkali feldspars. Radiation Measurements 27, 221-236.

Clarke, M. L., Botha, G. A., Wintle, A. G. and Vogel, J. C. Luminescence dating of Late Pleistocene South African colluvium from Voordrag, Kwa Zulu-Natal (in preparation)

David, B., Roberts, R., Tuniz, C., Jones, R. and Head, J. (1997) New optical and radiocarbon dates from Ngarrabullgan Cave, a Pleistocene archaeological site in Australia: implications for the comparability of time clocks and for the human colonization of Australia. Antiquity 71, 183-188.

Debenham, N. C. (1983) Reliability of thermoluminescence dating of stalagmitic calcite. Nature (London) $304,154-156$.
Debenham, N. C. (1987) Study of the effect of pre-annealing on sediment TL using a technique of glow curve analysis. Ancient $T L$ 5(1), 1-7.

Ditlefsen, C. and Huntley, D. J. (1994) Optical excitation of trapped charges in quartz, potassium feldspars and mixed silicates: the dependence on photon energy. Radiation Measurements 23, 675-682.

Duller, G. A. T. (1991) Equivalent dose determination using single aliquots. Nuclear Tracks and Radiation Measurements 18, 371-378.

Duller, G. A. T. (1994a) Luminescence dating using single aliquots: new procedures. Quaternary Geochronology (Quaternary Science Reviews) 13, 149-156.

Duller, G. A. T. (1994b) Luminescence dating of poorly bleached sediments from Scotland. Quaternary Geochronology (Quaternary Science Reviews) 13, $521-524$

Duller, G. A. T. (1995a) Infrared bleaching of the thermoluminescence of four feldspars. Journal of Physics D: Applied Physics 28, 1244-1258.

Duller, G. A. T. (1995b) Luminescence dating using single aliquots: methods and applications. Radiation Measurements 24, 217-226.

Duller, G. A. T. (1996) Recent developments in luminescence dating of Quaternary sediments. Progress in Physical Geography 20, 127-145.

Duller, G. A. T. (1997) Behavioural studies of stimulated luminescence from feldspars. Radiation Measurements 27, 663-694.

Duller, G. A. T. and Bøtter-Jensen, L. (1993) Luminescence from potassium feldspars stimulated by infrared and green light. Radiation Protection Dosimetry 47, 683-688.

Duller, G. A. T. and Bøtter-Jensen, L. (1996) Comparison of optically stimulated luminescence signals from quartz using different stimulation wavelengths. Radiation Measurements 26, 603-609.

Duller, G. A. T. and Bøtter-Jensen, L. (1997) Optically stimulated luminescence emission spectra from feldspars as a function of sample temperature. Radiation Measurements 27, 145-151.

Duller, G. A. T. and Wintle, A. G. (1991) On infrared stimulated luminescence at elevated temperatures. Nuclear Tracks and Radiation Measurements 18, 379 384.

Feathers, J. K. (1997) Luminescence dating of sediment samples from White Paintings Rock Shelter, Botswana. Quaternary Geochronology (Quaternary Science Reviews) 16, 321-331.

Fleming, S. J. (1970) Thermoluminescence dating: refinement of the quartz inclusion technique. Archaeometry 12, 133-145.

Fleming, S. J. (1973) The pre-dose technique: a new thermoluminescence dating method. Archaeometry 15, 13-30.

Forman, S. L., Lepper, K. and Pierson, J. (1994a) Limitations of infra-red stimulated luminescence in dating high arctic marine sediments. Quaternary Geochronology (Quaternary Science Reviews) 13, $545-550$.

Forman, S. L., Pierson, J., Smith, R. P., Hackett, W R. and Valentine, G. (1994b) Assessing the accuracy of thermoluminescence for dating baked sediments beneath late Quaternary lava flows, Snake River Plain, Idaho. Journal of Geophysical Research 99B(8), 15569-15576.

Fox, P. J. (1990) Optical studies of thermoluminescent materials. Ph.D. thesis, University of Adelaide.

Franklin, A. D. and Hornyak, W. F. (1990) Isolation of the rapidly bleaching peak in quartz TL glow curves. Ancient TL 8, 29-31. 
Franklin, A. D., Hornyak, W. F. and Dickerson, W. (1992) TL estimation of paleodose of dune-sand quartz. Quaternary Science Reviews 11, 75-78.

Franklin, A. D., Prescott, J. R. and Scholefield, R. B. (1995) The mechanism of thermoluminescence in an Australian sedimentary quartz. Journal of Luminescence 63, 317-326.

Fuller, I. C., Wintle, A. G. and Duller, G. A. T. (1994) Test of the partial bleach methodclogy as applied to the infra-red stimulated luminescence of an alluvial sediment from the Danube. Quaternary Geochronology (Quaternary Science Reviews) 13, 539-543.

Fuller, I. C., Macklin, M. G., Passmore, D. G., Brewer, P. A. Lewin, J. and Wintle, A. G. (1996) Geochronologies and environmental records of Quaternary fluvial sequences in the Guadalope basin, northeast Spain, based on luminescence dating. In: Global Continental Changes: the Context of Palaeohydrology (eds Branson, J., Brown, A. G. and Gregory, K. J.), pp 99-120. Geological Society Special Publication No. 115

Galloway, R. B. (1993) Stimulation of Juminescence using green light emitting diodes. Radiation Protection Dosimetry 47, 679-682.

Galloway, R. B. (1994) On the stimulation of luminescence with green light emitting diodes. Radiation Measurements 23, 547-550.

Galloway, R. B. (1996) Equivalent dose determination using only one sample: alternative analysis of data obtained from infrared stimulation of feldspars. Radiation Measurements 26, 103-106.

Garcia-Guinea, J., Rendell, H. M. and Sanchez-Munoz, L. (1996) Luminescence spectra of alkali feldspars: some relationships between struc.ural features and luminescence emission. Radiation Protection Dosimetry 66, 395-398.

Godfrey-Smith, D. I. (1994) Thermal effects in the optically stimulated luminescence of quartz and mixed feldspars from sediments. Journal of Physics D: Applied Physics 27, 1737-1746.

Godfrey-Smith, D. I. and Cada, M. (1996) IR stimulation spectroscopy of plagioclase and potassium feldspars, and quartz. Radiation Protection Dosimetry 66, 379385.

Godfrey-Smith, D. I., Huntley, D. J. and Chen, W.H. (1988) Optical dating studies of quartz and feldspar sediment extracts. Quaternary Science Review' 7, 373-380.

Grün, R. (1996) Errors in dose assessment introduced by the use of the "linear part" of a saturating dose response curve. Radiation Measurements 26, 297-302.

Grün, R. and McDonald, P. D. M. (1989) Non-linear fitting of TL/ESR dose-response: curves. Applied Radiation and Isotopes 40, 1077-1080.

Grün, R., Packman, S. C. and Pye, K.. (1989) Problems involved in TL-dating of Danish cover sands using K-feldspar. In: Long and Short Range Limits in Luminescence Dating, Occasional Publication No. 9 , pp. 13-18. Research Laboratory for Archaeology and the History of Art, Oxford.

Guibert, P., Vartanian, E., Bechtel, F. and Schvoerer, M. (1996) Non linear approach of TL response to dose: polynomial approximation. Ancient TL 14(2), $7-14$.

Hornyak, W. F., Chen, R. and Franklin, A. (1992) Thermoluminescence characterist cs of the $325^{\circ} \mathrm{C}$ electron trap in quartz. Physical Review B 46, 80368049.

Huntley, D. J. (1985) On the zeroing of the thermoluminescence of sediments. Physics and Chemistry of Minerals 12, 122-127.
Huntley, D. J. and Baril, M. R. (1997) The K content of the $\mathrm{K}$-feldspars being measured in optical dating or in thermoluminescence dating. Ancient $T L 15,11-13$.

Huntley, D. J. and Berger, G. W. (1995) Scatter in luminescence data for optical dating - some models. Ancient TL 13(1), 5-9.

Huntley, D. J. and Clague, J. J. (1996) Optical dating of tsunami-laid sands. Quaternary Research 46, 127140.

Huntley, D. J., Godfrey-Smith, D. I. and Haskell, E. H. (1991) Light-induced emission spectra from some quartz and feldspars. Nuclear Tracks and Radiation Measurements 18, 127-131.

Huntley, D. J., Godfrey-Smith, D. I. and Thewalt, M. L. W. (1985) Optical dating of sediments. Nature 313, $105-107$.

Huntley, D. J., Godfrey-Smith, D. I., Thewalt, M. L. W. and Berger, G. W. (1988a) Thermoluminescence spectra of some mineral samples relevant to thermoluminescence dating. Journal of Luminescence 39, $123-136$.

Huntley, D. J., Godfrey-Smith, D. I., Thewalt, M. L. W., Prescott, J. R. and Hutton, J. T. (1988b) Some quartz thermoluminescence spectra relevant to thermoluminescence dating. Nuclear Tracks and Radiation Measurements 14, 27-33.

Huntley, D. J., Hutton, J. T. and Prescott, J. R. (1993a) Optical dating using inclusions within quartz grains. Geology 21, 1087-1090.

Huntley, D. J., Hutton, J. T. and Prescott, J. R. (1993b) The stranded beach-dune sequence of south-east South Australia: A test of thermoluminescence dating, 0-800 ka. Quaternary Science Reviews 12, 1-20.

Huntley, D. J., Hutton, J. T. and Prescott, J. R. (1994) Further thermoluminescence dates from the dune sequence in the southeast of South Australia Quaternary Science Review's 13, 201-207.

Huntley, D. J., McMullan, W. G., Godfrey-Smith, D. I. and Thewalt, M. L. W. (1989) Time-dependent recombination spectra arising from optical ejection of trapped charges in feldspars. Journal of Luminescence 44, $41-46$.

Huntley, D. J., Short, M. A. and Dunphy, K. (1996) Deep traps in quartz and their use for optical dating. Canadian Journal of Physics 74, 81-91.

Hütt, G., Jaek, I. and Tchonka, J. (1988) Optical dating: $\mathrm{K}$-feldspars optical response stimulation spectra. Quaternary Science Reviews 7, 381-385.

Jungner, H. and Huntley, D. J. (1991) Emission spectra of some potassium feldspars under $633 \mathrm{~nm}$ stimulation. Nuclear Tracks and Radiation Measurements 18, 125126.

Kaylor, R. M., Feathers, J., Hornyak, W. F. and Franklin, A. D. (1995) Optically stimulated luminescence in Kalahari quartz: Bleaching of the $325^{\circ} \mathrm{C}$ peak as the source of the luminescence. Journal of Luminescence 65, 1-6.

Krbetschek, M. R., Götze, J., Dietrich, A. and Trautmann, T. (1997) Spectral information from minerals. Radiation Measurements 27, 695-748.

Krbetschek, M. R., Rieser, U. and Stolz, W. (1996) Optical dating: some dosimetric properties of natural feldspars. Radiation Protection Dosimetry 66, 407412.

Lamothe, M. and Auclair, M. (1997) Assessing the datability of young sediments by IRSL using an intrinsic laboratory protocol. Radiation Measurements 27, $107-117$.

Lamothe, M., Balescu, S. and Auclair, M. (1994) Natural IRSL intensities and apparent luminescence ages of single feldspar grains extracted from partially bleached sediments. Radiation Measurements 23, 555-561. 
Lang, A. and Wagner, G. A. (1997) Infrared stimulated luminescence dating of colluvial sediments using the $410 \mathrm{~nm}$ emission. Quaternary Geachronology (Quaternary Science Review's) 16, 393-396.

Li, S.-H. (1991) Removal of the thermally unstable signal in optical dating of K-feldspar. Ancient TL 9, 26-29.

Li, S.-H. (1994) Optical dating: insufficiently bleached sediments. Radiation Mectsurements 23, 563-567.

Li, S.-H. and Tso, M.-Y. W. (1997) Lifetime determination of OSL signals from potassium feldspar. Radiation Measurements 27, 119-121.

Li, S.-H. and Wintle, A. G. (1991) Sensitivity changes of luminescence signals from colluvial sediments after different bleaching procedures. Ancient $T L$ 9, 50-54.

Li, S.-H. and Wintle, A. G. (1992) A global view of the stability of luminescence signals from loess. Quaternary Science Review's 11, 133-137.

Li, S. H., Tso, M.-Y. and Wong, N. W. L. (1997) Parameters of OSL traps determined with various linear heating rates. Radiation Measurements 27, 43-47.

Liritzis, I. (1994) A new dating method by thermoluminescence of carved megalithic stone building. Comptes Rendus de l'Académie de.i Sciences Paris, serie II 319, 603-610.

Liritzis, I., Galloway, R. B. and Theocaris, P. S. (1994) Thermoluminescence dating of ceramics revisited: optical stimulated luminescence of quartz single aliquot with green light-ımitting diodes. Journal of Radioanalytical and Nuclear Chemistry 188(3), 189198.

Liritzis, I., Guibert, P., Foti, F. and Schvoerer, M. (1996) Solar bleaching of thermoluminescence of calcites. Nuclear Instruments and Methods in Physics Research B 117, 260-268.

Luff, B. J. and Townsend, F. D. (1993) High sensitivity thermoluminescence spectrometer. Engineering Optics 6, 149-156.

Lundqvist, J. and Mejdahl, V. (1987) Thermoluminescence dating of eolian sediments in central Sweden. Geologiska Foreningens $i$ Stockholm Forhandlingar $109,147-158$

McFee, C. J. and Tite, M. S. (1994) Investigations into the thermoluminescence projerties of single quartz grains using an imaging photon detector. Radiation Measurements 23, 355-350.

McKeever, S. W. S. (1991) Mechanisms of thermoluminescence production: some problems and a few answers. Nuclear Tracks and Radiation Measurements 18, 512.

McKeever, S. W. S. (1994) Models for optical bleaching of thermoluminescence in sediments. Radiation Measurements 23, 267-275.

McKeever, S. W. S. and Mcrris, M. F. (1994) Computer simulations of optical bleaching of TL and OSL signals. Radiation Measureinents 23, 301-306.

McKeever, S. W. S., Agersnip Larsen, N., Bøtter-Jensen, L. and Mejdahl, V. (1997) OSL sensitivity changes during single aliquot procedures: computer simulations. Radiation Meastrements 27, 75-82.

McKeever, S. W. S., Chen, C. Y. and Halliburton, L. E. (1985) Point defects and the pre-dose effect in natural quartz. Nuclear Tracks and Radiation Measurements 10, 489-495.

Mejdahl, V. (1985) Thermcluminescence dating of partially bleached sediments. Nuclear Tracks and Radiation Measurements 10, 711-715.

Mejdahl, V. (1988a) Long-term stability of the TL signal in alkali feldspars. Quaternary Science Reviews 7, $357-360$.

Mejdahl, V. (1988b) The plateau method for dating partially bleached sediments by thermoluminescence Quaternary Science Reviews 7, 347-348.
Mejdahl, V. (1989). How far back: Life times estimated from studies of feldspars of infinite ages. In: Long and Short Range Limits in Luminescence Dating, Occasional Publication No. 9, pp. 49-54. Research Laboratory for Archaeology and the History of Art, Oxford.

Mejdahl, V. and Bøtter-Jensen, L. (1994) Luminescence dating of archaeological materials using a new technique based on single aliquot measurements. Quaternary Geochronology (Quaternary Science Review's) 13, 551-554.

Mejdahl, V. and Bøtter-Jensen, L. (1997) Experience with the SARA OSL method. Radiation Measurements 27, 291-294.

Mejdahl, V. and Christiansen, H. H. (1994) Procedures used for luminescence dating of sediments. Quaternary Geochronology (Quaternary Science Review's) 13, 403-406.

Mejdahl, V., Shlukov, A. I., Shakhovets, S. A. Voskovskaya, L. T. and Lyashenko, H. G. (1992) The effect of shallow traps: a possible source of error in TL dating of sediments. Ancient TL 10(2), 22-25.

Morris, M. F. and McKeever, S. W. S. (1994) Optical bleaching studies of quartz. Radiation Measurements 23, 323-327.

Murray, A. S. (1996a) Incomplete stimulation of luminescence in young quartz sediments and its effect on the regenerated signal. Radiation Measurements 26, 221231.

Murray, A. S. (1996b) Developments in optically-stimulated luminescence and phototransferred thermoluminescence dating of young sediments: application to a 2,000-year sequence of flood deposits. Geochimica et Cosmochimica Acta 60, 565-576.

Murray, A. S. and Mejdahl, V. Comparison of regenerative-dose single-aliquot and multiple-aliquot (SARA) protocols using heated quartz from archaeological sites. Quaternary Geochronology (Quaternary Science Reviews) (submitted).

Murray, A. S. and Roberts, R. G. Determining the burial time of single grains of quartz using optically stimulated luminescence. Earth and Planetary Science Letters (in press a).

Murray, A. S. and Roberts, R. G. Measurement of the equivalent dose in quartz using a regenerative-dose single aliquot protocol. Radiation Measurements (in press b).

Murray, A. S. and Wintle, A. G. (1997) Factors controlling the shape of the OSL decay curve in quartz. Radiation Measurements (in press).

Murray, A. S., Olley, J. M. and Caitcheon, G. G. (1995) Measurement of equivalent doses in quartz from contemporary water-lain sediments using optically stimulated luminescence. Quaternary Geochronology (Quaternary Science Reviews) 14, 365-371.

Murray, A. S., Roberts, R. G. and Wintle, A. G. (1997) Equivalent dose measurement using a single aliquot of quartz. Radiation Measurements 27, 171-184.

Musson. F. M. and Wintle, A. G. (1994) Luminescence dating of the loess profile at Dolni Vestonice, Czech republic. Quaternary Geochronology (Quaternary Science Reviews) 13, 411-416.

Musson, F. M., Clarke, M. L. and Wintle, A. G. (1994) Luminescence dating of loess from the Liujiapo section, central China. Quaternary Geochronology (Quaternary Science Reviews) 13, 407-410.

Ollerhead, J., Huntley, D. J. and Berger, G. W. (1994) Luminescence dating of sediments from Buctouche Spit, New Brunswick. Canadian Journal of Earth Sciences 31, 523-531.

Olley, J. M., Caitcheon, G. G. and Murray, A. S. The distribution of apparent dose as determined by optically stimulated luminescence in small aliquots of fluvial 
quartz: implications for dating young sediments. Quaternary Geochronology (Quaternary Science Review's) (in press).

Olley, J. M., Roberts, R. G. and Murray, A. S. (1997) Disequilibria in the uranium decay series in sedimentary deposits at Allen's Cave, Nullarbor Plain, Australia: implications for dose riste determinations. Radiation Measurements 27, 433-4,13.

Page, K., Nanson, G. and Price, D. (1996) Chronology of Murrumbidgee River palaeochannels on the Riverine Plain, southeastern Australia. Journal of Quaternary Science 11, 31 $\mathbf{i}-326$.

Perkins, N. K. and Rhodes, E. J. (1994) Optical dating of fluvial sediments from Tattershall. U.K.. Quaternary Geochronology (Quaternary Science Review's) 13, $517-520$.

Petrov, S. A. and Bailiff, I. K. (1995) The " $110^{\circ} \mathrm{C} "$ TL peak in synthetic quartz. Radiation Measurements 24, $519-523$.

Poolton, N. R. J. and Bailiff, I. K. (1989) The use of LEDs as an excitation source for photoluminescence dating of sediments. Ancient $T L 7,18-20$.

Poolton, N. R. J., Bøtter-Jensen, L. and Duller, G. A. T. (1995) Thermal quenching of luminescence processes in feldspars. Radiation Measurements 24, 5766.

Poolton, N. R. J., Bøtter-Jensen, L. and Johnsen, O. (1996) On the relationship between luminescence excitation spectra and feldspar mineralogy. Radiation Measurements 26, 93-101.

Porat, N., Amit, R., Zilberman, E. and Enzel, Y. (1997) Luminescence dating of fault-related alluvial fan sediments in the Southern Arava Valley, Israel. Quaternary Geochronology (Quaternary Science Reviews) 16, 397-402.

Prescott, J. R. and Fox, P. J. (1990) Jating quartz sediments using the $325 \mathrm{C}$ TL peak: new spectral data. Ancient TL 8, 32-34.

Prescott, J. R. and Fox, P. J. (1993) Three-dimensional thermoluminescence spectra of fildspars. Journal of Physics D: Applied Physics 26, 22<5-2254.

Prescott, J. R. and Mojarrabi, B. (1953) Selective bleach: an improved partial bleach technique for finding equivalent doses for TL dating of quartz sediments. Ancient $T L$ 11(1), 27-30.

Prescott, J. R. and Robertson, G. B. (1997) Sediment dating by luminescence: a review. Radiation Measurements 27, 893-922.

Prescott, J. R., Akber, R. A. and Gartia, R. K. (1990) Three-dimensional thermoluminescence spectroscopy of minerals. Spectroscopic characterization of minerals and their surfaces, pp. 180-189. American Chemical Society, Washington D.C.

Prescott, J. R., Fox, P. J., Robertson, G. B. and Hutton, J. T. (1994) Three-dimensional spectral studies of the bleaching of the thermoluminescence of feldspars. Radiation Measurements 23, 367-375.

Price, D. M. (1994) TL dating of fluvial quartz sands: a comparison of ages obtained at $325^{\circ} \mathrm{C}$ and $375^{\circ} \mathrm{C}$. Ancient TL 12(2), 20-23.

Pye, K., Stokes, S. and Neal, A. (1995) Optical dating of aeolian sediments from the Sefton coast, northwest England. Proceedings of the Geologists' Association 106, 281-292.

Questiaux, D. G. (1991) Optical dating of loess: comparisons between different grain size fractions for infrared and green excitation wavelengths. Nuclear Tracks and Radiation Measurements 18, 33-139.

Rendell, H. M., Lancaster, N. and Tchakerian, V. P. (1994a) Luminescence dating of Late Quaternary aeolian deposits at Dale Lake and Cronese Mountains, Mojave Desert, California. Quaternary
Geochronology (Quaternary Science Reviews) 13, 417-422.

Rendell, H. M., Townsend, P. D. and Wood, R. A. (1995) TL and IRSL emission spectra of detrital feldspars; new experimental data. Physica Status Solidi (b) 190 , $321-330$.

Rendell, H. M., Webster, S. E. and Sheffer, N. L. (1994b) Underwater bleaching of signals from sediment grains: new experimental data. Quaternary Geochronology (Quaternary Science Reviews) 13, 433-435.

Rendell, H. M., Worsley, P., Green, F. and Parks, D. (1991) Thermoluminescence dating of the Chelford Interstadial. Earth and Planetary Science Letters 103, 182-189.

Rhodes, E. J. (1988) Methodological considerations in the optical dating of quartz. Quaternary Science Reviews $7,395-400$.

Richardson, C. A. (1994) Sensitivity changes due to bleaching of the infra-red stimulated luminescence of potassium-rich feldspars: effects on the dating of sands from the Mojave Desert, California. Ph.D. thesis, University of Wales, Aberystwyth.

Richardson, C. A., McDonald, E. V. and Busacca, A. J. (1997) Luminescence dating of loess from the north west United States. Quaternary Geochronology (Quaternary Science Review's) 16, 403-415.

Roberts, R., Walsh, G., Murray. A.. Olley, J., Jones, R., Morwood, M., Tuniz, C., Lawson, E., Macphail, M. Bowdery, D. and Naumann, I. (1997) Luminescence dating of rock art and past environments using mudwasp nests in northern Australia. Nature 387, 696699.

Roberts, R. G. (1997) Luminescence dating in archaeology: from origins to optical. Radiation Measurements 27, 819--892.

Roberts, R. G., Jones, R., Spooner, N. A., Head, M. J., Murray, A. S. and Smith, M. A. (1994a) The human colonisation of Australia: Optical dates of 53,000 and 60,000 years bracket human arrival at Deaf Adder Gorge, Northern Territory. Quaternary Geochronology (Quaternary Science Reviews) 13, $575-583$.

Roberts, R. G., Spooner, N. A. and Questiaux, D. G. (1993) Cautions on the use of extended duration preheats in the optical dating of quartz. Ancient $T L$ 11(2), 47-54.

Roberts, R. G., Spooner, N. A. and Questiaux, D. G. (1994b) Palaeodose underestimates caused by extended-duration preheats in the optical dating of quartz. Radiation Measurements 23, 647-653.

Robertson, G. B., Prescott, J. R. and Hutton, J. T. (1991) Bleaching of the thermoluminescence of feldspars by sunlight. Nuclear Tracks and Radiation Measurements 18, $101-107$.

Robertson, G. B., Prescott, J. R. and Hutton, J. T. (1993) Bleaching of the thermoluminescence of feldspars by selected wavelengths present in sunlight. Nuclear Tracks and Radiation Measurements 21, 245-251.

Scholefield, R. B., Prescott, J. R., Franklin, A. D. and Fox, P. J. (1994) Observations on some thermoluminescence emission centres in geological quartz. Radiation Measurements 23, 409-412.

Shlukov, A. I. and Shakhovets, S. A. (1987) Kinetic studies of quartz thermoluminescence as applied to sediment dating. Ancient TL 5(1), 11-15.

Shlukov, A. I., Shakhovets, S. A., Voskovskaya, L. T. and Lyashenko, M. G. (1993) A criticism of standard TL dating technology. Nuclear Instruments and Methods in Physics Research 73, 373-381.

Shlukov, A. I.. Usova. M. G., Shakhovets, S. A. and Voskovskaya, L. T. (1997) New techniques of absol- 
ute dating of Quaternary sediments. world wide web http/www.aha.ru/ shluk ov!

Short, M. A. and Huntley, L. J. (1992) Infrared stimulation of quartz. Ancient $T L$ 10, 19-21.

Short, M. A. and Tso, M.-Y. W. (1994) New methods for determining the thermal activation energies of light sensitive traps. Radiation Measurements 23, 335-338.

Smith, B. W. (1983) New applications of thermoluminescence dating and comparisons with other methods. Ph.D. thesis, University of Adelaide.

Smith. B. W. (1988) Zircons from sediments: a combined OSL and TL auto-regenerative dating technique. Quaternary Science Reviews 7, 401-406.

Smith, B. W., Aitken, M. J., Rhodes, E. J., Robinson, P. D. and Geldard, D. M. (1986) Optical dating: Methodological aspects. Radiation Protection Dosimetry 17, 229-233.

Smith, B. W., Rhodes, E. J., Stokes, S. and Spooner, N. A. (1990a) The optical dating of sediments using quartz. Radiation Protection Dosimetry 34, 75-78.

Smith, B. W., Rhodes, E. J., Stokes, S., Spooner, N. A. and Aitken, M. J. (1990b) Optical dating of sediments: Initial quartz results from Oxford Archaeometry 32, 19-31

Smith, B. W., Wheeler, G. C. W. S., Rhodes, E. J. and Spooner, N. A. (1991) Luminescence dating of zircon using an imaging photon detector. Nuclear Tracks and Radiation Measurements 18, 273-278.

Smith. M. A., Prescott, J. R. and Head, M. J. (1997) Comparison of ${ }^{14} \mathrm{C}$ and luminescence chronologies at Puritjarra Rock Shelter, Central Australia. Quaternary Geochronology' (Quaternary Science Reviews) 16, 299-320.

Spooner, N. A. (1992) Optical dating: Preliminary results on the anomalous fading of luminescence from feldspars. Quaternary Science Reviews 11, 139-145.

Spooner, N. A. (1994a) The anomalous fading of infraredstimulated luminescence from feldspars. Radiation Measurements 23, 625-632.

Spooner, N. A. (1994b) On the optical dating signal from quartz. Radiation Measurements 23, 593-600.

Spooner, N. A. and Franks, M. (1990) Some characteristics of infrared emitting diodes relevant to luminescence dating. Ancient TL 8, 16-19.

Spooner, N. A. and Questiaux., D. G. (1989). Optical dating - Achenheim beyond the Eemian using green and infra-red stimulation. In: Long and Short Range Limits in Luminescence Dating, pp. 97-103. Occasional Publication No. 9. Research Laboratory for Archaeology and the History of Art, Oxford.

Spooner, N. A., Prescott, J. R. and Hutton, J. T. (1988) The effect of illumination wavelength on the bleaching of the thermoluminescence (TL) of quartz. Quaternary Science Reviews 7, 325-329.

Stokes, S. (1992) Optical dating of young (modern) sediments using quartz: results from a selection of depositional environments. Quaternary Science Reviews 11, 153-159.

Stokes, S. (1994) The timing of OSL sensitivity changes in a natural quartz. Radiaion Measurements 23, 601605.

Stokes, S. (1996a) Further comparisons of quartz OSL additive dose palaeodoses generated using long and short duration preheats. Ancient $T L$ 14(1), 1-3.

Stokes, S. (1996b) Pre-heats, palaeodoses and paradigms in the optical dating of quartz. Ancient $T L$ 14(1), 58.

Stokes, S., Kocurek, G., Fye, K. and Winspear, N. R. (1997) New evidence for the timing of aeolian sand supply to the Algodones dunefield and East Mesa area, southestern California, USA. Palaeogeography, Palaeoclimatology, Palaeoecology 128, 63-75.
Stoneham, D. and Stokes, S. (1991) An investigation of the relationship between the $110^{\circ} \mathrm{C}$ TL peak and optically stimulated luminescence in sedimentary quartz. Nuclear Tracks and Radiation Measurements 18, 119-123.

Strickertsson, K. (1985) The thermoluminescence of potassium feldspars - glow curve characteristics and initial rise measurements. Nuclear Tracks and Radiation Measurements 10, 613-617.

Templer, R. (1985) The dating of zircons by auto-regenerated TL at low temperatures. Nuclear Tracks and Radiation Measurements 10, 789-798.

Templer, R. H. (1986) Auto-regenerative TL dating of zircon inclusions. Radiation Protection Dosimetry 17, 235-239.

Templer, R. H. and Smith, B. W. (1988) Auto-regenerative TL dating with zircon from fired materials. Nuclear Tracks and Radiation Measurements 14, 329-332.

Tso, M.-Y. W. and Li, S.-H. (1994) Equivalent dose estimation for pottery by single disc regeneration method. Radiation Measurements 23, 451-454

Tso, M.-Y. W., Wong, N. W. L. and Li, S. H. (1996) Determination of lifetime of infrared stimulated signals from potassium and sodium feldspars. Radiation Protection Dosimetry 66, 387-389.

Ugumori, T. and Ikeya, M. (1980) Luminescence of $\mathrm{CaCO}_{3}$ under $\mathrm{N}_{2}$ laser excitation and application to archaeological dating. Japanese Journal of Applied Physics 19, 459-465.

Valladas, G. and Valladas, H. (1982) Influence du débit de dose sur la thermoluminescence du quartz (II). PACT 6, 281-291.

Visocekas, R. (1985) Tunnelling radiative recombination in labradorite; its association with anomalous fading of thermoluminescence. Nuclear Tracks and Radiation Measurements 10, 521-529.

Visocekas, R. (1993) Tunnelling radiative recombination in K-feldspar sanidine. Nuclear Tracks and Radiation Measurements 21, 175-178.

Wintle, A. G. (1973) Anomalous fading of thermoluminescence in mineral samples. Nature $245,143-144$.

Wintle, A. G. (1975) Thermal quenching of thermoluminescence in quartz. Geophysical Journal of the Royal Astronomical Society 41, 107-113.

Wintle, A. G. (1978) A thermoluminescence dating study of some Quaternary calcite: potential and problems. Canadian Journal of Earth Sciences 15, 1977-1986.

Wintle, A. G. (1982) Thermoluminescence properties of fine-grain minerals in loess. Soil Science 134, 164170.

Wintle, A. G. (1990) A review of the current research on TL dating of loess. Quaternary Science Review's 9, 385-397.

Wintle, A. G. and Huntley, D. J. (1979) Thermoluminescence dating of a deep-sea sediment core. Nature 279, 710-712.

Wintle, A. G. and Huntley, D. J. (1980) Thermoluminescence dating of ocean sediments. Canadian Journal of Earth Sciences 17, 348-360.

Wintle, A. G. and Murray, A. S. (1997) The relationship between quartz thermoluminescence, photo-transferred thermoluminescence, and optically stimulated luminescence. Radiation Measurements 27, 611-624.

Wintle, A. G. and Murray, A. S. Towards the development of a preheat procedure for OSL dating of quartz. Radiation Measurements (in press).

Wintle, A. G., Botha, G. A., Li, S.-H. and Vogel, J. C. (1995a) A chronological framework for colluviation during the last $110 \mathrm{kyr}$ in KwaZulu/Natal South African Journal of Science 91, 134-139.

Wintle, A. G., Clarke, M. L., Musson, F. M., Orford, J. D. and Devoy, R. J. N. Luminescence dating of 
recent dunes on Inch Spit, Dingle Bay, Southwest Ireland. The Holocene (in press).

Wintle, A. G., Li, S.-H., Botha, G. A. and Vogel, J. C. (1995b) Evaluation of lumineszence dating procedures applied to late-Holocene colluvium near St Paul's Mission, Natal, South Africa. The Holocene 5 , 97-102.

Wood, P. B. (1994) Optically stimulated luminescence dating of a late Quaternary shoreline deposit, Tunisia. Quaternary Geochronology (Quaternary Science Reviews) 13, 513-516.

Yang, X. H. and McKeever, S. W. S. (1990) The predose effect in crystalline quartz. Journal of Physics $D$. Applied Physics 23, 237-244.
Zhou, L. P. and Wintle, A. G. (1994) Sensitivity change of thermoluminescence signals after laboratory optical bleaching: experiments with loess fine grains. Quaternary Geochronology (Quaternary Science Reviews) 13, 457-463.

Zimmerman, J. (1971) The radiation-induced increase of thermoluminescence sensitivity of fired quartz. Journal of Physics C: Solid State Physics 4, 32773291.

Zink, A. and Visocekas, R. (1996) Dosimetry with feldspars and their infrared emission band. Radiation Protection Dosimetry 66, 399-402.

Zink, A. J. C. and Visocekas, R. (1997) Datability of sanidine feldspars using the near-infrared TL emission. Radiation Measurements 27, 251-261. 